\title{
Efeito de Rotação nos Fluxos Zonais e Modos Acústicos Geodésicos
}

Aluno: Reneé Jordashe Franco Sgalla

Orientador: Prof. Dr. Ricardo Magnus Osório Galvão

13 de abril de 2010 


\section{DEDICATÓRIA}

Dedico este trabalho à minha namorada, Mila Silva Costa, aos meus pais, Remo Sgalla e Maria Alice Franco Sgalla, e a todos que, assim como estas pessoas, se propõem a ajudar os seus semelhantes sem esperar benefícios em troca. 


\section{AGRADECIMENTOS}

Esta tese é o resultado de praticamente três anos de muito trabalho e dedicação. Durante esse período, contei com a ajuda de algumas pessoas e instituições, às quais devo minha sincera gratidão.

Devo muito ao meu orientador, o professor Dr. Ricardo Magnus Osório Galvão, pela paciência e disposição ao me orientar durante o programa de mestrado. Agradeço também por ter me apresentado e dado boas referências minhas ao prof. Dr. J. P. Hans Goedbloed.

Sou grato também ao professor Dr. J. P. Hans Goedbloed pelas aulas de Magnetohidrodinâmica por ele ministradas no Instituto de Astronomia e Geofísica da Universidade de São Paulo no início de 2007. Também devo a ele meus sinceros agradecimentos pela carta de recomendação que me possibilitou participar do "Eigth Carolus Magnus Summer School on Plasma and Fusion Energy Physics" realizado em Bad Honnef na Alemanha em setembro de 2007, em que aprendi muito, tanto do ponto de vista acadêmico, quanto do ponto de vista pessoal.

Meus agradecimentos ao prof. Dr. Artour Elfimov por me ajudar a compreender artigos importantes relacionados com o meu trabalho e pelas sugestões e ideias, as quais contribuíram significativamente para este trabalho.

Agradeço ao apoio financeiro fornecido pela FAPESP e pela CNPq, sem os quais, não poderia me manter no período em que estive no mestrado.

Durante grande parte do mestrado, enfrentei problemas pessoais com os quais tive muita dificuldade em lidar. Devo minhas mais sinceras gratidão aos meus pais, Remo Sgalla e Maria Alice Franco Sgalla, ao meu tio, Euclides Luiz Sgalla, e à minha namorada, Mila Silva Costa, que além de me apoiarem nas horas mais difíceis, também acreditaram em mim e me ajudaram a superar as dificuldades que enfrentei. Em especial, agradeço a Mila Silva Costa pela revisão ortográfica e gramatical desta dissertação. 


\section{Resumo}

Investigamos o efeito da rotação de equilíbrio nos fluxos zonais (ZF) e modos acústicos geodésicos (GAM) em tokamaks de secção circular. Estes modos, ZF e GAM, ocorrem em sistemas toroidais como uma resposta do plasma à curvatura geodésica das linhas de campo magnético e devido ao movimento de deriva do fluido (plasma). Este movimento de deriva é causado pela resposta de partículas carregadas ao campo elétrico e magnético e, para ambas as espécies de cargas, isto é, íons positivos e elétrons, o movimento é na mesma direção.

Ao fazer uso de basicamente três aproximações - tokamaks de alta razão de aspecto, princípio de quasi-neutralidade e baixos valores de $\beta$ (aproximação eletrostática) - e ao perturbar as equações da magnetohidrodinâmcia ideal até primeira ordem, constatamos que a rotação de equilíbrio, de fato, afeta a frequência dos ZF e dos GAM.

No equilíbrio com rotação toroidal, já investigado por W. Shaojie, os ZF se tornam instáveis e a frequência dos GAM se altera com a rotação no caso de condutividade de calor finita; entretanto, quando a condutividade de calor tende a infinito, a rotação não influencia na frequência dos ZF e GAM. Esta observação e os valores das frequências dos ZF e GAM diferem da publicação original e portanto, se estivermos corretos, nosso trabalho poderá ajudar a resolver o problema dos ZF e dos GAM, que são questões ainda não completamente entendidas.

A rotação causada por um campo elétrostático que surge devido à difusão ambipolar, ainda não investigada anteriormente, é descrita neste trabalho. Vimos que não há instabilidades neste caso, porém este tipo de rotação também afeta a frequência dos ZF e dos GAM. A frequência dos ZF, que na ausência de rotação é nula, é proporcional à intensidade do fluxo de rotação. Interpretamos este resultado como uma consequência do efeito doppler. A frequência dos GAM, por outro lado, se anula quando a rotação atinge um certo valor, que está relacionado com o fator de segurança e a razão de aspecto do tokamak.

Considerando de ordem um o fluxo de rotação, obtivemos altas frequências, as quais não são aceitáveis em nosso modelo devido à aproximação de quasi-neutralidade, de forma que nosso modelo é válido apenas para fluxos cuja ordem equivalem à razão de aspecto inversa. Entretanto, mesmo neste regime, a rotação tem grande impacto na frequência dos ZF e GAM. Este é o principal resultado obtido. 


\begin{abstract}
We investigate the efect of equilibrium rotation on zonal flows (ZF) and geodesic acoustic modes (GAM) in tokamaks of circular cross section. These modes, ZF and GAM, occur in toroidal systems as a response of the plasma to the geodesic curvature of the magnetic field lines and due to the drift motion of the fluid (plasma). This drift motion is caused by the response of charged particles to the electric and magnetic field and, for both species of charges, i. e., positive ions and negative electons, the motion is in the same direction.

By making use of basically three aproximations, high aspect ratio tokamkas, the quasi-neutrality principle and low $\beta$ values (eletrostatic approximation), and perturbing the ideal magnetohidrodynamics equations to first order, we find that the equilibrium rotation does afects the ZF and GAM frequencies.

In the equilibrium toroidal rotation flow, which had already been studied by W. Shaojie, the ZF becomes unstable and the GAM frequency is changed by rotation for finity heat conductivity; but when the heat conductivity goes to infinity, the rotation has no influence on the ZF and GAM frequencies. This assertion and also the ZF and GAM frequencies values differ from the original publication and therefore, if we are correct, our work may help to solve the ZF and GAM problem, which is not yet a completely understood subject.

The rotation caused by an equilibrium electostatic field created by ambipolar diffusion, which has not been dealt is also investigate. We find no instability in this case, but the rotation also affects the ZF and GAM frequencies. The ZF frequency, which is usually null, becomes proportional to the equilibrium rotation flow. We interptret this result as a concequency of the doppler effect. The GAM frequency, on the other hand, becomes zero when the rotation reaches a certain value, which is related to the safety factor and the aspect ratio of the tokamak.

Consideration of equilibrium flows scaling to order one leads to high frequencies, which is not accepted by contrasting the quasi-neutrality approximation and so, our model is valid only for small equilibrium electric field that scale to the inverse aspect ratio. But even in this regime, we find that the rotation has great impact on the ZF and GAM frequencies, which is the main result we obtain.
\end{abstract}




\section{Sumário}

1 Introdução $\quad 1$

1.1 Energia para futuras gerações . . . . . . . . . . . . . . . . 1

1.2 Obtenção de energia por meio de fusão nuclear . . . . . . . . . . . . . . 2

1.3 Tokamak ........................... 4

1.4 Transporte turbulento e supressão por meio de ZF . . . . . . . . . . . . 7

1.5 Objetivos, resultados e organização da dissertação . . . . . . . . . . . 8

2 Supressão de transporte turbulento por meio de ZF e GAM - Uma $\begin{array}{ll}\text { discussão qualitativa } & \mathbf{1 1}\end{array}$

3 Equações da Magneto-hidrodinâmica $\quad 15$

3.1 MHD ideal aplicada aos ZF e GAM . . . . . . . . . . . . . . . . . 17

3.2 Equações da MHD ideal perturbadas em primeira ordem . . . . . . . . . 19

4 Equilíbrio MHD 23

4.1 Equilíbrio em uma dimensão . . . . . . . . . . . . . . . . . . . . 25

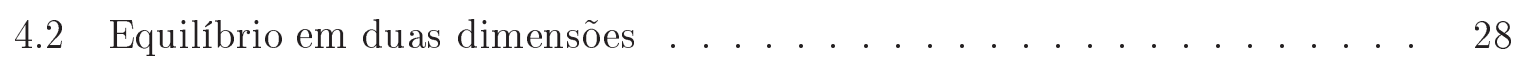

4.2.1 ENR e a equação de Grad-Shafranov . . . . . . . . . . . . . . . 30

4.2.2 Equilíbrio com rotação $(\mathrm{ER})$. . . . . . . . . . . . . . 33

4.3 Discussão sobre EBRF . . . . . . . . . . . . . . . . . . 37

4.4 Sumário sobre equilíbrio . . . . . . . . . . . . . . . . . 38

5 Física básica dos ZF e GAM 41

5.1 Ondas acústicas em geometria retangular . . . . . . . . . . . . 41

5.2 GAM em sistemas toroidais . . . . . . . . . . . . . . . . . . 44

6 Efeito da rotação nos ZF e GAM em tokamaks de secção circular 53

6.1 Equilíbrio sem rotação - ENR . . . . . . . . . . . . . . . 57

6.2 Equilíbrio com rotação toroidal - ETRF . . . . . . . . . . . . . . . 59 
6.3 Equilíbrio com rotação binormal - EBRF . . . . . . . . . . . . . . . 62

7 Conclusões e propostas para trabalhos futuros $\quad 66$

$\begin{array}{ll}\text { Referências Bibliográficas } & 70\end{array}$

$\begin{array}{ll}\text { Apêndices } & 72\end{array}$

$\begin{array}{ll}\text { A Identidades vetoriais } & 72\end{array}$

B Sistemas de coordenadas $\quad \mathbf{7 5}$

B.1 Sistema de coordenada arbitrário . . . . . . . . . . . . . . . . 75

B.2 Coordenadas cilíndricas globais . . . . . . . . . . . 76

B.3 Coordenadas pseudo-toroidais . . . . . . . . . . . . 77

B.4 Coordenadas cilíndricas locais . . . . . . . . . . . . . . 77

B.5 Relação entre coordenadas cilíndricas e coordenadas pseudo-toroidais . . 78

B.6 Derivativos de vetores . . . . . . . . . . . . . . 79

C Função de fluxo e resolução da equação de Grad-Shafranov $\quad 81$

C.1 Função $\Psi_{0}$ e o campo magnético $\boldsymbol{B}_{0}$ (uma dimensão) . . . . . . . . . . . 81

C.2 Função $\Psi$ e o campo magnético $\boldsymbol{B}$ (duas dimensões) . . . . . . . . . . . . 82

C.3 Solução da equação de Grad-Shafranov . . . . . . . . . . . . . . . . . . . 82

D Sistema de coordenadas $\left(\hat{\boldsymbol{e}}_{\Psi}, \hat{\boldsymbol{e}}_{\|}, \hat{\boldsymbol{e}}_{\perp}\right) \quad 88$

D.1 Versores e operadores vetoriais . . . . . . . . . . . . . . 88

D.2 Ortogonalidade de funções seno-cossenoidais . . . . . . . . . . . . . . . . . . . . . . 91

D.3 Operadores aplicados a funções seno-cossenoidais . . . . . . . . . . . 92

E Cálculo do determinante do capítulo $6 \quad 96$

F Lista de símbolos e siglas utilizados ao longo da dissertação $\quad 99$

F.1 Lista dos principais símbolos matemáticos . . . . . . . . . . . . . 99

F.2 Lista de siglas . . . . . . . . . . . . . . . . . . . . . . 101 


\section{Lista de Figuras}

1.1 Esquema básico de um tokamak: 1 - Bobinas do enrolamento primário do tokamak, 2 - Coluna de plasma, 3 - Eixo magnético (região de maior pressão e densidade), 4 - Corrente toroidal (corrente de plasma), 5 - Cấmara de vácuo, 6 - Campo magnético poloidal $\left(B_{\theta}\right), 7$ - Borda da coluna de plasma, 8 - Campo magnético toroidal $\left(B_{\phi}\right), 9$ - Bobinas toroidais, 10 - Núcleo de material ferromagnético, 11 - Banco de capacitores, 12 Bobina responsável pelo campo magnético vertical. . . . . . . . . . .

1.2 Sistemas de coordenadas utilizadas para descrever o tokamak (secção circular): $(R, \varphi, Z)$ - coordenadas cilíndricas globais, $(r, \theta, z)$ - coordenadas cilíndricas locais, $(r, \theta, \phi)$ - coordenadas pseudo-toroidais. . . . . . . . .

1.3 Secções transversal de um tokamak. A esquerda é mostrada a secção circular, a qual é definida pelos parâmetros $R_{0}$ e $a$. A direita é mostrada a secção em forma de "D", que além de depender dos parâmetros $R_{0}$ e $a$, também depende de $\delta, K$ e $b \ldots \ldots \ldots \ldots$

1.4 Supressão de vórtices turbulentos por meio de ZF. . . . . . . . . . . . 9

4.1 Equilíbrio em uma dimensão . . . . . . . . . . . . . . . . 26

4.2 Na superfície do plasma $Z=0, B_{\theta}=B_{Z}$, de forma que é conveniente escolher esta superfície para calcular o fluxo magnético poloidal. . . . . .

4.3 Deslocamento de Shafranov $\left(\Delta_{S}\right)$ : Secção transversal de um tokamak de secção circular no qual são mostradas as superfícies magnéticas. . . . . . .

5.1 Aproximação local das coordenadas pseudo-toroidais para coordenadas cartesianas ............................. 42

5.2 Grandezas físicas relevantes para a descrição dos GAM no tokamak . . . 51 


\section{Capítulo 1}

\section{Introdução}

\subsection{Energia para futuras gerações}

A crescente demanda por energia em nosso planeta coloca em cheque o futuro da humanidade, o qual também depende de fatores de natureza tecnológica, ambiental e política. A maior parte da energia gasta no consumo humano (80 a $90 \%$ ) é obtida por meio da queima de combustíveis fósseis, os quais estão fadados à escassez em um futuro não muito distante (30 a 40 anos para o caso do petróleo). [1]

O consumo de energia médio no planeta é de aproximadamente 15 TW ano; entretanto estima-se que daqui a aproximadamente 50 anos, com o aumento da população e do consumo humano, ele será aproximadamente o dobro do atual [1, 2, 3]. Com base nestas estimativas, espera-se que nas próximas décadas enfrentemos problemas nunca antes vistos envolvendo uso de energia.

É possível que conflitos políticos - tais como a guerra do Golfo (1991) e da Chechenia, e crises de energia como as da década de 70 - sejam apenas uma pequena escala dos problemas decorrentes da escassez de energia, os quais enfrentaremos se providências a respeito não forem tomadas no curto prazo. Neste terrível cenário ainda não consideramos as consequências ambientais e industriais ocasionadas pelo uso de combustíveis fósseis, embora elas já começem a mostrar sinais de sua existência. Dados da quantidade de $\mathrm{CO}_{2}$ contida na atmosfera mostram um enorme crescimento nas últimas décadas [4] causando um aumento da temperatura média global, o que certamente irá alterar o ecosistema e possivelmente tornará algumas partes do mundo inabitáveis devido ao aumento do nível do mar e formação de desertos, gerando fome, pobreza e, consequentemente, ameaçando a paz mundial. Além disso, toda essa matéria prima, utilizada para geração de energia e posteriormente lançada na atmosfera na forma de $\mathrm{CO}_{2}$, é de inestimável valor para a indústria química e farmacêutica. 
Tendo em vista o que foi dito acima, não faltam argumentos para afirmar que a busca por novas fontes de energia a base de combustíveis não fósseis é imprescindível para a sobrevivência humana no futuro. Entre as possíveis alternativas conhecidas temos recursos renováveis (biocombustíveis, energia eólica, energia solar, etc..), fissão nuclear e fusão nuclear.

Por outro lado, embora os combustíveis renováveis sejam abundantes e não estejam sujeitos à escassez no futuro, eles são limitados em sua capacidade de produzir energia e estão sujeitos a variações naturais, o que requer armazenagem por períodos de tempo, gerando, assim, custos financeiros. Ademais, devido às suas limitações de produção, eles só poderão ser utilizados para complementar outros meios de produção de energia.

No caso da fissão nuclear, dejetos altamente radioativos são produzidos; porém uma boa parte destes podem ser processados e reutilizados em outros reatores. Entretanto o problema da escassez no futuro ainda não pode ser descartado. Afinal os atuais reatores a fissão em funcionamento esgotarão as reservas de urânio em cerca de 50-60 anos. Além disso, problemas ambientais e de segurança, que não são facilmente solúveis e a difícil aceitação do público geral, ainda são obstáculos para o uso difundido deste tipo de energia. [1]

Já no caso da fusão nuclear, os problemas de limitação de produção, de escassez, de consequências ambientais e de segurança são superados e, mais do que isto, trata-se de uma fonte de energia limpa e de larga escala, mantendo-se assim o compromisso com a preservação ambiental e as necessidades humanas simultaneamente. Estudos recentes realizados pela Comissão Européia confirmam este ponto de vista [5]. Em contrapartida, a utilização de fusão nuclear para gerar energia é uma das tarefas mais complexas do ponto de vista científico e técnico e, por isso, meios para se conseguir esta tarefa estão sendo estudado por cientistas e pesquisadores no mundo inteiro.

\subsection{Obtenção de energia por meio de fusão nuclear}

A fusão termonuclear controlada tem se mostrado o meio mais eficaz de obter energia em escalas suficientes para o consumo humano no longo prazo. A reação nuclear capaz de produzir a máxima energia a mais baixa temperatura dos reagentes é aquela entre Deutério (D) e Trítio (T),

$$
\mathrm{D}+\mathrm{T} \rightarrow{ }^{4} \mathrm{He}(3,5 \mathrm{MeV})+\mathrm{n}(14,1 \mathrm{MeV})
$$


na qual os produtos da reação são particulas $\alpha\left({ }^{4} \mathrm{He}\right)$ e nêutrons altamente energéticos $(n)$. Mesmo para esta reação, a temperatura do meio reagente deve ser da ordem de $10^{8}$ $\mathrm{K}[1]$.

Atualmente existem duas linhas de pesquisa em fusão nuclear: fusão inercial e fusão magnética. Na primeira, o intuito é realizar fusão dirigindo feixes de laser ou partículas para pequenas esferas contendo combustível nuclear em seu interior [6]. Na segunda, fusão magnética, que tem se mostrada a linha mais promissora até o presente momento, objetiva-se o confinamento do combustível nuclear por meios de campos magnéticos. Dentre os dispositivos de confinamento magnético, que têm sido investigados - tokamaks, stellarators e descargas de estrição com campo reverso ${ }^{1}$ - o tokamak [7] é o que tem mostrado os melhores resultados e perspectivas para o futuro, de forma que descrevemos apenas este dispositivo neste trabalho.

No tokamak, o mecanismo de produção de energia é o seguinte: um plasma ${ }^{2}$ de isótopos de hidrogênico ( $\mathrm{D}$ e $\mathrm{T}$ ), por exemplo, é confinado e mantido a temperaturas suficientemente elevadas por meio de campos magnéticos criados auto-consistentemente por correntes que circulam no próprio plasma e por correntes que circulam em bobinas externas, até que reações de fusão entre os íons do plasma comecem a ocorrer. Estas reações liberam nêutrons altamente energéticos que são moderados em um manto externo à câmara de vácuo que contém o plasma, produzindo, assim, grande quantidade de calor que, por sua vez, é transformado em energia elétrica ${ }^{3}$.

Por meio da fusão nuclear é possível converter uma pequena quantidade de matéria (massa $m$ ) em uma enorme quantidade de energia, $E$, de acordo com a famosa equação de Einstein,

$$
E=m c^{2}
$$

onde $c \approx 3,0.10^{8} \mathrm{~ms}^{-1}$ é a velocidade da luz no vácuo. A matéria convertida em energia provém dos íons do combustível nuclear ( $\mathrm{D}$ e $\mathrm{T}$ ). Uma simples estimativa comparativa de 1.2 e 1.1 com valores conhecidos do rendimento de combustíveis fósseis e outros combustíveis nucleares estabelece a relação de aproximadamente $250 \mathrm{Kg}$ de Deutério e Trítio para 1.900.000 toneladas de petróleo (queima de combustíveis fósseis) ou 28 toneladas de Urânio (fissão nuclear) para a mesma potência de 1000 MW [1].

\footnotetext{
${ }^{1} \mathrm{O}$ nome em ingles é "Reversed Field Pinches"

${ }^{2}$ A grosso modo, o plasma é um gás totalmente ionizado cujos elétrons e íons (positivos) não estão ligados entre si em sua maioria. A estabilidade do plasma é possível se, após a ionização inicial, o número de recombinações for próximo ao número de ionizações geradas por fontes externas de energia.

${ }^{3} \mathrm{Um}$ dos métodos para essa transformação consiste em utilizar o calor para aquecer água até a formação de vapor e utilizar esse vapor para movimentar a turbina de um gerador elétrico.
} 


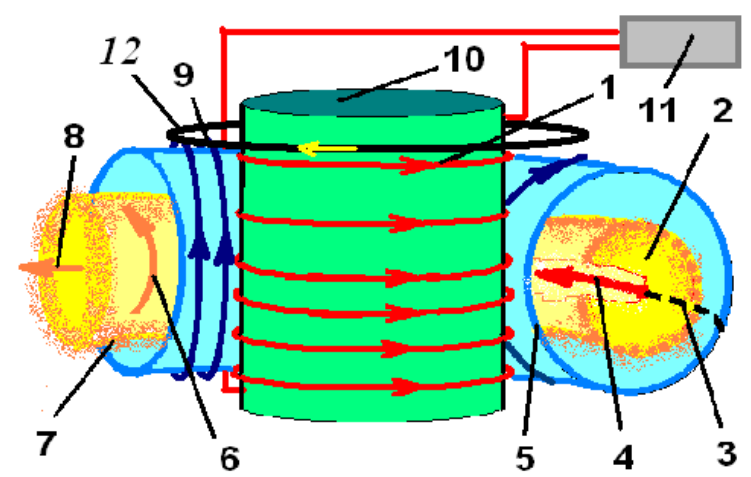

Figura 1.1: Esquema básico de um tokamak: 1 - Bobinas do enrolamento primário do tokamak, 2 - Coluna de plasma, 3 - Eixo magnético (região de maior pressão e densidade), 4 - Corrente toroidal (corrente de plasma), 5 -Câmara de vácuo, 6 - Campo magnético poloidal $\left(B_{\theta}\right), 7$ - Borda da coluna de plasma, 8 - Campo magnético toroidal $\left(B_{\phi}\right), 9-$ Bobinas toroidais, 10 - Núcleo de material ferromagnético, 11 - Banco de capacitores, 12 - Bobina responsável pelo campo magnético vertical.

\subsection{Tokamak}

O tokamak ${ }^{4}$ é a configuração mais simples, porém mais eficaz, para o confinamento magnético do plasma. O esquema de um tokamak típico pode ser visto na figura 1.1.

O plasma é confinado pela combinação dos campos magnéticos toroidal, $B_{\phi}(8)$, e poloidal, $B_{\theta}(6)$. O primeiro é criado pelas bobinas de campo toroidal (9) que são colocadas ao longo da câmara de vácuo contendo o plasma (5), enquanto o segundo surge em decorrência da corrente toroidal $(4)^{5}$. Esta corrente, por sua vez, surge no plasma devido à baixa resistividade deste e em decorrência do campo elétrico toroidal $\left(E_{T} \approx 0,5\right.$ $\mathrm{V} / \mathrm{m}$ para o tokamak TCABR) induzido no interior da câmara de vácuo quando as bobinas do enrolamento primário (1) são percorridas por uma corrente elétrica variável provinda da descarga de um banco de capacitores (11). O tokamak é uma espécie de transformador, em que a coluna de plasma atua como enrolamento secundário (2) e as bobinas (1), como enrolamento primário. O campo magnético vertical criado pela bobina (12) tem a função de impedir que a coluna de plasma, como um todo, sofra um aumento de seu raio maior e se desloque, assim, em sentido à parte externa da câmera de vácuo. Isso ocorreria devido à força magnética que surge em pontos opostos da coluna de plasma em virtude de circularem correntes (corrente de pasma) em sentidos contrários

\footnotetext{
${ }^{4} \mathrm{~A}$ palavra tokamak é um acrônimo russo para câmera toroidal magnética.

${ }^{5}$ Também conhecida como corrente de plasma $-I_{p}$.
} 


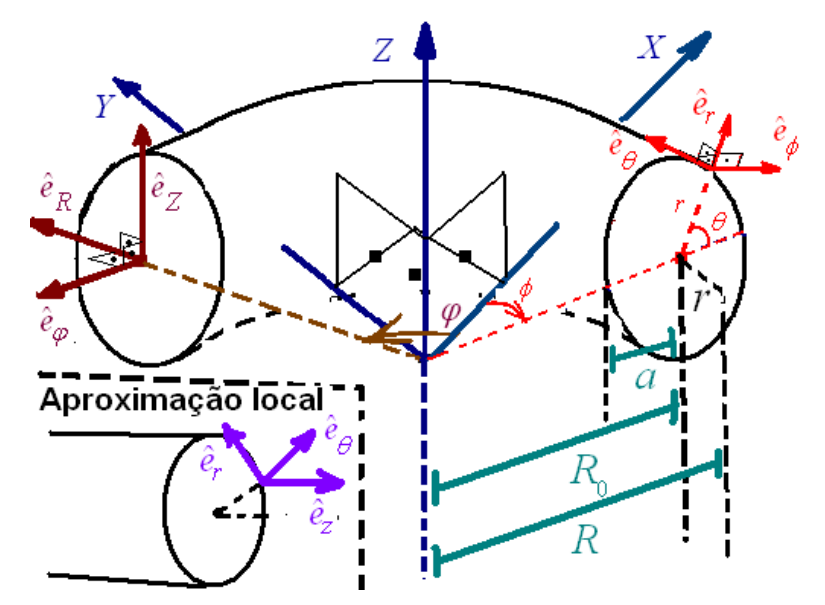

Figura 1.2: Sistemas de coordenadas utilizadas para descrever o tokamak (secção circular): $(R, \varphi, Z)$ - coordenadas cilíndricas globais, $(r, \theta, z)$ - coordenadas cilíndricas locais, $(r, \theta, \phi)$ - coordenadas pseudo-toroidais.

nestes pontos. Neste trabalho desconsideramos este efeito e portanto não consideramos o campo magnético vertical, cuja descrição mais detalhada pode ser vista em [9].

Em um reator a fusão nuclear em funcionamento, a câmara seria revestida internamente por uma manta contendo Lítio, o qual funcionaria como uma fonte de Trítio para o plasma ao ser atingidos por nêutrons energéticos provenientes do plasma. Essa reação exotérmica é capaz de fornecer uma grande quantidade de calor que poderia ser utilizada para aquecer a água no interior de uma tubulação próxima à manta de Lítio produzindo, assim, vapor a alta pressão capaz de movimentar uma turbina, a qual poderia acionar um gerador elétrico, por exemplo. Em tokamaks de secção circular o eixo magnético (3) está localizado no centro da coluna de plasma; é nesta região que a pressão hidrostática e a densidade têm seus valores máximos. Tal região é de difícil acesso para diagnósticos por ter uma temperatura extremamente alta. Por outro lado, é na borda da coluna de plasma (7) que são colocados todas as sondas e dispositivos responsáveis pelo diagnóstico. É nesta região que ocorre a detecção dos fluxos zonais $\left(\mathrm{ZF}^{6}\right)$ e dos modos acústicos geodésicos (GAM), que são investigados neste trabalho.

A figura 1.2 mostra o tokamak do ponto de vista geométrico. Apresentamos os sistemas de coordenadas mais utilizados na descrição física de tokamaks: coordenadas cilíndricas globais, $(R, \varphi, Z)$, coordenadas cilíndricas locais, $(r, \theta, z)$ e as coordenadas pseudo-toroidais, $(r, \theta, \phi)$.

Em muitos tokamaks a seção transversal não é circular, ao contrário do tokamak

${ }^{6} \mathrm{ZF}-$ Zonal Flows (fluxos zonais) 


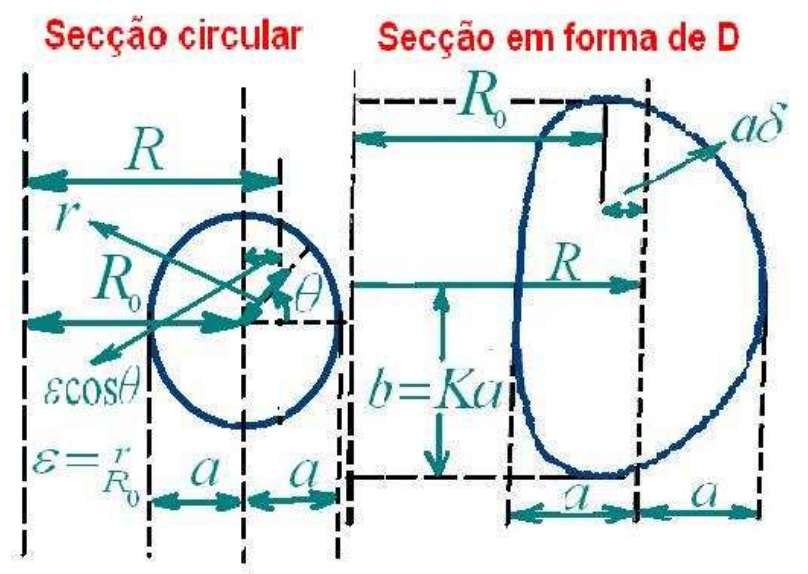

Figura 1.3: Secções transversal de um tokamak. A esquerda é mostrada a secção circular, a qual é definida pelos parâmetros $R_{0}$ e $a$. A direita é mostrada a secção em forma de "D", que além de depender dos parâmetros $R_{0}$ e $a$, também depende de $\delta, K$ e $b$.

mostrado na figura 1.2. Este é o caso do ITER $[8]^{7}$ e provavelmente será o caso dos futuros reatores a fusão nuclear. Tais secções possuem os seguintes parâmetros de configuração: Razão de aspecto, $A=R_{0} / a$; elongação, $K=b / a$; e triangularidade, $\delta$, conforme mostra a figura 1.3. Tal secção não-circular, também conhecida como secção em forma de D, são utilizadas com o intuito de conseguir maior estabilidade no plasma [15].

Para que tenhamos um ganho efetivo de energia ${ }^{8}$, é necessário realizar a difícil tarefa de confinar e isolar um plasma com densidade da ordem de $10^{20} \mathrm{~m}^{-3}$ e energia térmica da ordem de $10 \mathrm{keV},{ }^{9}$ suficientes para que ocorra um determinado número de reações de fusão capaz de fornecer uma quantidade de energia maior do que aquela gasta com perdas e com o processo de ignição do plasma. Além disso, o tempo de confinamento deve ser grande o suficiente para alimentar dispositivos auxiliares essenciais para manter o reator em funcionamento [1].

O desenvolvimento de fusão magnética como uma fonte comercial de eletricidade requer a solução de muitos desafios físicos, os quais são tradicionalmente separados em três categorias: equilíbrio e estabilidade, aquecimento e transporte. Na primeira categoria, o objetivo principal é encontrar configurações magnéticas que possibilitem manter o plasma em condições de estabilidade macroscópica [9]. No estudo de estabilidade, procura-se des-

\footnotetext{
${ }^{7}$ ITER - International Thermonuclear Experimental Reactor (Reator termonuclear experimental internacional) - Primeiro protótipo de um reator a fusão que está sendo construído em Cadarache na França, com previsão para término em 2014.

${ }^{8}$ Definido como a diferença entre a energia obtida com a fusão do combustível nuclear pela energia gasta para manter o plasma confinado de forma estável.

${ }^{9}$ Equivalente a temperaruras de $10^{8} \mathrm{~K}$
} 
crever os modos de oscilações temporais das principais grandezas físicas de interesse e classificar esses modos de acordo com seus valores de frequência [10]. No que se refere aos processos de aquecimento e transporte em plasmas, objetiva-se desenvolver regimes de confinamento que minimizem perdas e maximizem a eficiência do confinamento [11].

\subsection{Transporte turbulento e supressão por meio de ZF}

O estudo de transporte começou desde os primórdios da teoria cinética, a qual foi desenvolvida com o intuito de explicar fenômenos fora do equilíbrio em gases a partir de primeiros princípios. ${ }^{10}[12]$. Após o desenvolvimento da teoria de transporte para gases neutros, foram feitas tentativas para adaptá-la para plasmas de fusão. Nasceu então a famosa teoria de transporte clássico para plasmas [13]. Entretanto o valor dos coeficientes de transporte previsto por essa teoria eram muito inferiores aos revelados por experimentos, muitas vezes por várias ordens de grandeza. Uma melhora significativa para as previsões dos coeficientes de transporte veio com a teoria de transporte neoclássico [14], que leva em conta tanto a natureza dos processos de colisões como o efeito da inomogeneidade do campo magnético sobre as órbitas das partículas. Entretanto, em muitos casos, mesmo a teoria neoclássica falha em explicar os altos valores dos coeficientes de transporte observados experimentalmente. Neste caso, o transporte é anômalo e ainda não há uma teoria muito bem fundamentada para descrever este tipo de transporte [12].

A explicação mais plausível para explicar o transporte anômalo é de que um plasma real nunca se apresenta em um estado quiescente ${ }^{11}$, como considerado pela teoria neoclássica. As partículas agem sincronizadamente organizando-se de forma a criarem ondas, modos ou até mesmo vórtices devido à natureza coletiva causada pela forças coulombianas de longo alcance. Elas formam assim estruturas coerentes que são muito mais eficientes em transportar matéria e energia do que individualmente. Quando as amplitudes dos modos são suficientemente pequenas, as interações entre elas resultam em processos relativamente simples, como, por exemplo, a união de duas ondas transformando-se em uma nova onda, de forma que ainda podemos tratar o problema linearmente. Entretanto pode ocorrer que a amplitude das ondas comece a aumentar consideravelmente, em uma escala de tempo característica, surge então uma instabilidade. Neste caso o problema já não pode ser tratado linearmente. Há um crescimento da amplitude até um ponto em que surja uma grande quantidade de modos ou até que haja uma saturação das amplitudes dos modos. Assim a evolução do plasma se torna complexa e imprevisível, ou seja, o

\footnotetext{
${ }^{10}$ Isto é, da visão microscópica, ou visão molecular da matéria.

${ }^{11}$ Isto é, inativo, estável.
} 
plasma se torna turbulento (ou caótico). Em certos regimes a contribuição proveniente da dissipação da turbulência é significativamente maior do que as contribuições clássicas e neoclássicas provenientes das colisões das partículas do plasma; estamos lidando, então, com transporte anômalo, para o qual ainda não há uma teoria consistente e bem fundamentada. [12]

Apesar de não haver uma teoria bem definida para explicar o transporte anômalo, descobriu-se que, em um determinado regime de operação, uma forte barreira se forma a uma distância do centro da coluna de plasma [11]. Nesta barreira o transporte turbulento de matéria e energia para a borda da coluna de plasma diminui consideravelmente. Tal regime foi denominado modo $\mathrm{H}^{12}$ em contraposição com o antigo regime de funcionamento (modo $\mathrm{L}^{13}$ ). A redução da turbulência (e consequentemente do transporte) nesta barreira permite aumentar o tempo de confinamento do plasma. Tal regime de funcionamento será em princípio indispensável para os futuros reatores a fusão [8].

A supressão de turbulência nesta barreira se baseia na destruição de células convectivas, ou vórtices turbulentos, conforme mostra na figura 1.4. Essas células são advectadas ${ }^{14}$ por fluxos cizalhados ${ }^{15}$, em particular os ZF. Ao serem advectadas com diferentes velocidades e em diferentes partes, estas começam a se alongar até a perda de correlação, fragmentando-se, então, em vórtices menores. O tempo de duração das novas estruturas (vórtices) é uma fração do tempo de duração do vórtice original e consequentemente a turbulência é reduzida. A formação dos ZF se deve a presença de campos elétricos e campos magnético do plasma. Os fluxos cizalhados existentes no plasma, geralmente, são separados em duas categorias distintas, os ZF, com frequência nula e os $\mathrm{GAM}^{16}$ ), com frequência da ordem da frequência de ondas acústicas [16, 17].

Devido a sua capacidade de reduzir a turbulência e consequentemente o transporte, os ZF e os GAM têm sido alvo de intenso interesse entre a comunidade científica.

\subsection{Objetivos, resultados e organização da dissertação}

Nosso intuito é descrever, do ponto de vista teórico, a dinâmica dos ZF e dos GAM preservando a clareza e a concisão. Utilizamos para isso o modelo da MHD ideal, que, embora não possa descrever muitos dos fenômenos em física de plasma, tem a grande vantagem de ser relativamente simples. Sempre que necessário utilizamos a aproximação

\footnotetext{
${ }^{12}$ Modos H - High confinement modes (modos de alto confinamento)

${ }^{13}$ Modos L - Low confinement modes (modos de baixo confinamento.

${ }^{14}$ Levadas por fluxos existentes no plasma (ZF).

${ }^{15}$ Fluxos cuja velocidade varia conforme a posição perpendicular a seu deslocamento

${ }^{16} \mathrm{GAM}$ - Geodesic acoustic modes (modos acústicos geodésicos)
} 


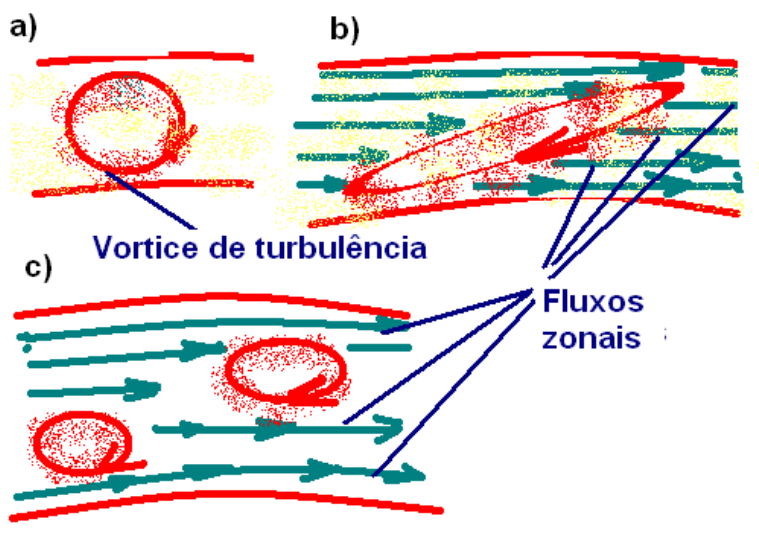

Figura 1.4: Supressão de vórtices turbulentos por meio de ZF.

de alta razão de aspecto que, embora possa restringir a validade dos resultados, permite sua derivação analítica e facilita a compreensão física dos fenômenos tratados. Esperamos, assim, proporcionar um trabalho que sirva de base para pesquisadores iniciando seus estudos de transporte turbulento em plasmas de laboratório. Contudo, novos resultados e críticas de trabalhos já publicados também estão presentes nesta dissertação, permitindo, então, que pesquisadores que já tenham conhecimento no assunto também se beneficiem.

Investigamos o efeito da rotação de equilíbrio nos ZF e nos GAM. Para isto, utilizamos, além das equações da MHD, a teoria de perturbações temporais, que permite linearizar as equações da MHD resolvendo-as analiticamente para encontrar a relação de dispersão para os ZF e GAM.

Embora diversos artigos sobre ZF e GAM tenham sido publicados, não encontramos nenhum artigo que tratasse de forma clara e concisa os mecanismos físicos por trás da formação destes. Nem tão pouco, encontramos artigos que descrevessem o efeito da rotação causada pelo movimento de deriva do plasma em equilíbrio, o qual decorre da existência de um campo elétrico radial na borda da coluna de plasma.

Os resultados obtidos mostram que a rotação toroidal $\left(\mathrm{ETRF}^{17}\right)$ faz com que os ZF se tornem instáveis e diminui a frequência dos GAM, contribuindo, assim, ao nosso ver, negativamente para a redução de transporte turbulento. Já a rotação binormal $\left(\mathrm{EBRF}^{18}\right)$, a qual é provocada pelo campo elétrico ambipolar de equilíbrio, faz com que os ZF tenham uma frequência de oscilação, cujo surgimento atribuímos ao efeito doppler

\footnotetext{
${ }^{17}$ ETRF - Equilibrium toroidal rotation flow (fluxo de rotação toroidal de equilíbrio)

${ }^{18} \mathrm{EBRF}$ - Equilibrium binormal rotation flow (fluxo com rotação binormal de equilíbrio)
} 
causado pela rotação de equilíbrio. Por outro lado, os GAM, que na ausência de rotação de equilíbrio têm frequência positiva, apresentam frequências que variam conforme a intensidade da rotação de equilíbrio, podendo, inclusive, ter frequência nula. Apesar de não causar instabilidades nos ZF e GAM, a rotação binormal leva a novos resultados que não foram previstos antes.

A dissertação é organizada da seguinte forma: no capítulo 2, descrevemos de forma qualitativa o mecanismo de supressão de transporte turbulento por meio de ZF e GAM; no capítulo 3, apresentamos o modelo da magneto-hidrodinâmcia (MHD) que iremos utilizar no restante do trabalho; no capítulo 4, investigamos o equilíbrio MHD do plasma com rotação, o qual é a base para entender os capítulos 5 e 6 . O capítulo 5 descreve o mecanismo físico dos ZF e GAM utilizando o equilíbrio sem rotação ( $\operatorname{ENR}^{19}$ ) e o sexto capítulo trata do efeito da rotação $\left(\mathrm{ER}^{20}\right)$ nesses modos. Finalmente apresentamos a conclusão e propostas para trabalhos futuros. Sempre que conveniente, deixamos para apresentar as derivações extensas nos apêndices A-E.

\footnotetext{
${ }^{19} \mathrm{ENR}$ - Equilibrium with no rotation (equilíbrio sem rotação)

${ }^{20} \mathrm{ER}$ - Equilibrium with rotation (equilíbrio com rotação)
} 


\section{Capítulo 2}

\section{Supressão de transporte turbulento por meio de ZF e GAM - Uma discussão qualitativa}

Há cerca de sessenta anos David Bohm mostrou empiricamente que , devido a processos turbulentos, o coeficiente de difusão em plasmas confinados magneticamente é da forma

$$
D=\frac{T_{e}}{16 e B} \approx 6,25 \times 10^{6} \frac{T_{e}}{B} \quad\left(\mathrm{~cm}^{2} \mathrm{~s}^{-1}\right)
$$

onde $T_{e}$ é a temperatura do gás de elétrons, e é a carga elementar do elétron e $B$ é o campo magnético do plasma [18]. Ao contrário do que previa a teoria cinética clássica de transporte colisional [13], em que o coeficiente é da forma

$$
D \propto \frac{1}{\sqrt{T} B^{2}}
$$

De 2.1 e 2.2 vemos que o coeficiente de transporte obtido por Bohm é muito superior ao esperado pela teoria clássica de transportes [13] e, ainda, de acordo com 2.1, a expressão para o coeficiente de transporte anômalo é muito menos conveniente do que o previsto por 2.2 , visto que o coeficiente de transporte depende diretamente da temperatura, de forma que, ao aumentar a temperatura para melhorar o confinamento, o transporte também aumenta, contribuindo, assim, negativamente para o confinamento. Por este motivo, métodos capazes de reduzir o transporte anômalo têm importância fundamental para a 
melhoria do confinamento do plasma.

Um regime de particular importância, no qual o tempo de confinamento é pelo menos o dobro do observado em descargas convencionais, é o modo $\mathrm{H}^{1}$ [19]. Nesse regime, uma barreira de transporte caracterizada por um forte gradiente de pressão se forma na borda da coluna de plasma. Posteriormente, descobriram-se novos regimes de confinamento nos quais a barreira de transporte se forma no interior da coluna de plasma, em regiões em que o cizalhamento das linhas de força do campo magnético é reduzido [20]. Tais regimes serão de fundamental importância para a melhoria do confinamento no ITER [21].

Recentemente, notou-se que, em fluxos turbulentos, é possível a transferência de energia entre a turbulência de pequena escala e fluxos médios macroscópicos, principalmente através do tensor de Reinolds, $\tau=<\tilde{\boldsymbol{v}} \tilde{\boldsymbol{v}}>$, onde $\tilde{\boldsymbol{v}}$ é a velocidade perturbada associada à turbulência [11, 23]. A correlação implícita no tensor de Reynolds dá origem aos fluxos zonais (ZF), que são células de convecção sem variação na direção azimutal, mas com alternância de sentido na direção radial [23, 24]. A formação de fluxos zonais foi confirmada em vários experimentos e, atualmente, eles constituem um modelo teórico paradigmático para explicar a formação de barreiras de transporte [26].

O transporte turbulento pode ser consideravelmente reduzido na presença dos ZF. O mecanismo de supressão de transporte, proposto por P. W. Terry [11], é o seguinte: o cizalhamento dos ZF (variação transversal da velocidade do fluxo) faz com que os vórtices do fluido turbulento sejam esticados e deformados devido ao fato de que diferentes partes de cada vórtice estão sujeitos à ação dos fluxos cizalhados de diferentes velocidades. Dizemos assim que as diferentes partes do vórtice são advectadas (levadas ao longo do fluido) com diferentes velocidades.

Um único vórtice isolado pode ser esticado em várias vezes seu comprimento inicial sem se partir (perder coerência); entretanto, quando este vórtice é parte de um fluido turbulento, ao ser esticado além de um determinado tamanho $\left(L_{c}\right.$ - comprimento de coerência), ele se parte em estruturas menores. O comprimento de coerência, $L_{c}$, é estimado como a distância entre dois vórtices adjacentes de tamanhos próximos e, em casos de turbulência completamente desenvolvida, $L_{c}$ mede aproximadamente o diâmetro de um vórtice. Na ausência de ZF, o tempo necessário para a perda de correlação é o tempo de vida do vórtice, $\tau_{t}^{2}$, que é dimensionalmente igual ao seu período de rotação. Na presença de $\mathrm{ZF}$, este tempo é reduzido e, consequentemente, $\tau_{t}$ também é reduzido. O decréscimo de $\tau_{t}$ implica na redução da intensidade da turbulência, devido à taxa de dissipação de energia exceder a taxa de fornecimento de energia para a turbulência, levando assim a

\footnotetext{
${ }^{1}$ Modos $\mathrm{H}$ - High Confinement modes - Modos de alto confinamento

${ }^{2} \tau_{t}$ - Turnover time - Período de rotação
} 
um novo balanço de energia mais baixa. A taxa de dissipação da turbulência é definida como a razão entre a energia da turbulência e o tempo de correlação $\left(\tau_{t}\right)$. Além disso, devido ao alongamento dos vórtices ao longo da direção do fluxo, as parcelas dos vórtices se deslocam apenas uma fração do seu diâmetro inicial se não houvesse a perda de correlação. Este deslocamento é ao longo da direção de cizalhamento (radial) e, portanto, a sua diminuição reduz também o livre caminho médio do passeio aleatório, neste processo de transporte radial. Esta redução de transporte radial ocorre principalmente em uma região conhecida como barreira de transporte, cuja descoberta tornou possível melhorar o rendimento do confinamento, o qual era severamente limitado pela turbulência de pequena escala.

A descoberta da formação de barreiras de transporte possibilitou um enorme avanço na tecnologia de confinamento de plasmas. Nos modos $\mathrm{H}^{3}$, consegue-se reduzir bastante o nível de turbulência que se propaga a partir do centro da coluna de plasma e, consequentemente, também se reduz o nível de transporte. No futuro, espera-se que seja possível obter regimes ainda melhores, nos quais as barreiras de transporte se formem mais próximas do centro da coluna de plasma. Com isso seria possível reduzir ainda mais o transporte anômalo.

É interessante notar que, ao destruir as estruturas de vórtices turbulentos provenientes das ondas de deriva [25], o cizalhamento dos fluxos faz com que a componente radial do vetor de onda ${ }^{4}$ aumente como consequência da fragmentação das estruturas originais. Como consequência, a frequência das ondas de deriva diminuem e, com ela, a sua energia. Como a energia total deve ser conservada, esta diminuição de energia das ondas de deriva causa, então, um aumento da energia dos ZF. Portanto, surge uma instabilidade que deve chegar a uma saturação em algum momento. O resultado global é o aparecimento de um novo estado em que o nível de turbulência e, consequentemente, de transporte anômalo radial é reduzido significativamente, melhorando, assim, o confinamento.

Toda esta discussão qualitativa, que atualmente é o modelo mais utilizado para descrever o mecanismo de redução do transporte anômalo por ZF, não leva em consideração o efeito da rotação do plasma, mesmo a chamada rotação residual, que é causada por efeitos térmicos e ocorre em praticamente todos os regimes experimentais. Como esta rotação não é rígida, mas apresenta um perfil radial, é de se esperar que ela possa interagir com os ZF ou GAM e, assim, tenha efeitos importantes na redução da turbulência no plasma.

Nesta dissertação damos um primeiro passo no estudo desse mecanismo, analisando

\footnotetext{
${ }^{3}$ Regime em que ocorre a formação de barreiras de transporte.

${ }^{4} \mathrm{O}$ vetor de onda radial é estimado como o inverso do comprimento de correlação, $k_{r}=1 / L_{c}$.
} 
o efeito da rotação na relação de dispersão desses modos, a qual depende, além da taxa de rotação do equilíbrio, do fator de segurança, definido por

$$
q(r)=\frac{r B_{\phi}}{R_{0} B_{\theta}}
$$

e da velocidade do som no plasma, $c_{s}$.

Uma outra grandeza de extrema importância em plasmas de fusão, a qual voltará a aparecer neste trabalho (capítulo 4), é o parâmetro $\beta$, definido como a razão entre a pressão cinética, $p$, e a pressão magnética, $B^{2} /\left(2 \mu_{0}\right)$,

$$
\beta=\frac{2 \mu_{0} p}{B^{2}}
$$

Consideramos o regime de baixos valores de $\beta$, o qual nos permite supor o campo magnético constante no tempo. Para $\beta$ alto, estaremos lidando com os ZF e GAM eletromagnéticos. 


\section{Capítulo 3}

\section{Equações da Magneto-hidrodinâmica}

A Magneto-hidrodinâmica (MHD) ideal é o modelo mais simples para determinar o equilíbrio macroscópico e as propriedades de estabilidade de plasmas confinados magneticamente. O modelo descreve como forças magnéticas, inerciais e de pressões interagem em um plasma perfeitamente condutor disposto em uma geometria magnética arbitrária. Há um consenso geral de que a configuração geométrica de um reator a fusão deve obedecer limites de equilíbrio e estabilidade estabelecidos pela MHD ideal, caso contrário o confinamento do plasma terminaria em um curto período de tempo (se comparado com tempos experimentais). Sendo assim o principal objetivo do estudo da MHD em plasmas de fusão é a descoberta de configurações magnéticas que possibilitem equilíbrio atrativo e estabilidade em reatores a fusão.

Este modelo fornece uma descrição do comportamento macroscópico do plasma, o qual é considerado como sendo constituído de um ou mais fluidos cuja dinâmica obedece aos princípios da mecânica dos fluidos. Este modelo também considera as interações eletromagnéticas entre os fluidos e os campos eletromagnéticos externos e internos (gerados consistentemente no plasma).

As equações da MHD podem simplesmente ser postuladas a partir de princípios físicos ou obtidas calculando-se os momentos de diversas ordens da equação de Boltzmann, pela teoria cinética [10]. Na derivação a partir da teoria cinética, supõe-se que o plasma é altamente colisional, o que quase nunca é satisfeito em plasmas de fusão; entretanto há muitas evidências empíricas de que o modelo da MHD descreve com uma boa precisão o comportamento macroscópico do plasma em muitos fenômenos de interesse [9].

Para obter as equações da MHD, é necessário combinar as equações de Maxwell com as equações que descrevem a dinâmica dos fluidos e ultilizar a equação que descreve a interação entre elas. As equações de Maxwell descrevem a evolução do campo elétrico $\boldsymbol{E}(\boldsymbol{r}, t)$ e do campo magnético $\boldsymbol{B}(\boldsymbol{r}, t)$ em resposta à densidade de carga, $\tau(\boldsymbol{r}, t)$, e de 
corrente elética, $\boldsymbol{J}(\boldsymbol{r}, t)$. Tais equações são dadas por

$$
\begin{gathered}
\nabla \cdot \boldsymbol{E}=\frac{\tau}{\varepsilon_{0}}, \\
\nabla \cdot \boldsymbol{B}=0, \\
\nabla \times \boldsymbol{E}=-\frac{\partial \boldsymbol{B}}{\partial t} \quad \mathrm{e} \\
\nabla \times \boldsymbol{B}=\mu_{0} \boldsymbol{J}+\frac{1}{c^{2}} \frac{\partial \boldsymbol{E}}{\partial t} .
\end{gathered}
$$

Enquanto a equação 3.1 expressa a lei de Gauss e permite o cálculo do campo elétrico a partir da quantidade de monopolos elétricos, a equação 3.2 nos diz que, também no plasma, não há monopolos magnéticos. A equação 3.3 é a tradução matemática da lei de Faraday, e a equação 3.4 traduz a lei de Ampère com a adição da corrente de deslocamento introduzida por Maxwell, que, conforme mostraremos, pode ser desprezada no limite de velocidades não relativísticas, quando tratamos de fenômenos de baixas frequências.

As equações da dinâmica dos gases expressam a evolução da densidade de massa $\rho(\boldsymbol{r}, t)$ e da pressão, $p(\boldsymbol{r}, t)$ a partir do conhecimento da velocidade do fluido $\boldsymbol{v}(\boldsymbol{r}, t)$. Elas são dadas por

$$
\begin{aligned}
& \frac{D \rho}{D t}+\rho \boldsymbol{\nabla} \cdot \boldsymbol{v}=0 \quad \mathrm{e} \\
& \frac{D p}{D t}+\gamma p \boldsymbol{\nabla} \cdot \boldsymbol{v}=0,
\end{aligned}
$$

onde $\frac{D}{D t}$ é a derivada Lagrangiana, dada por

$$
\frac{D}{D t}=\frac{\partial}{\partial t}+\boldsymbol{v} \cdot \boldsymbol{\nabla}
$$


em que $\frac{\partial}{\partial t}$ é a derivada Euleriana, a qual deve ser calculada em uma posição $\boldsymbol{r}$ fixa, e $\gamma=C_{p} / C_{v}$ é a razão das capacidades térmicas a pressão e a volume constantes, $C_{p}$ e $C_{v}$. As equações 3.5 e 3.6 traduzem a conservação de massa e de energia (entropia) respectivamente, que são válidas na ausência de fontes e/ou sorvedouros de matéria ou de calor.

A interação entre o fluido e o campo eletromagnético é dada pela equação de movimento, que expressa a primeira lei de Newton, e é dada por

$$
\rho \frac{D \boldsymbol{v}}{D t}=\boldsymbol{F}
$$

onde $\boldsymbol{F}$ é a densidade de força que age no plasma. Para o caso particular de plasmas de laboratório,

$$
\boldsymbol{F}=-\nabla p+\boldsymbol{J} \times \boldsymbol{B}+\tau \boldsymbol{E} .
$$

Por fim temos a equação que expressa a relação entre o campo elétrico em um referencial que se move com o fluido e a densidade de corrente neste fluido, também conhecida por lei de Ohm,

$$
\boldsymbol{E}^{\prime}=\eta \boldsymbol{J}
$$

onde $\boldsymbol{E}^{\prime}=\boldsymbol{E}+\boldsymbol{v} \times \boldsymbol{B}$ é o campo elétrico no referencial do fluido e $\eta$ é a resistividade do plasma.

As equações 3.1 a 3.10 constituem um sistema completo de 16 equações e 16 incógnitas, as três componentes vetoriais de $\boldsymbol{E}, \boldsymbol{B}, \boldsymbol{J}$ e $\boldsymbol{v}$, e as grandezas escalares $\tau, \rho, p$ e $\eta$. O fato de o número de equações ser igual ao número de incógnitas nos permite em princípio descrever completamente o plasma no regime de validade da MHD.

\subsection{MHD ideal aplicada aos ZF e GAM}

No estudo de ZF e GAM, podemos considerar, com base em dados experimentais [26], que a velocidade do fluxo de plasma é da ordem da velocidade do som, $c_{s}$, de forma que se trata de velocidades não relativísticas, ou seja, $v / c \ll 1$, e termos de ordem $O\left(v^{2} / c^{2}\right)$ podem ser desprezados. Sendo assim podemos desprezar a corrente de deslocamento de Maxwell em 3.4, de acordo com a seguinte análise da ordem de grandeza, 


$$
\begin{aligned}
\nabla \Longrightarrow[L]^{-1}, \quad \frac{\partial}{\partial t} \Longrightarrow[T]^{-1}, \quad E \Longrightarrow[L][T]^{-1} B \quad \text { e } \quad[L][T]^{-1} & \Longrightarrow v \\
\frac{\left\|\frac{1}{c^{2}} \frac{\partial \boldsymbol{E}}{\partial t}\right\|}{\|\boldsymbol{\nabla} \times \boldsymbol{B}\|} & \sim \frac{v^{2}}{c^{2}}
\end{aligned}
$$

onde $L$ é o comprimento característico do plasma e $T$ é o período característico de oscilação dos modos. Além disso, as frequências dos ZF e GAM são $\omega_{z, n r} \sim 0$ e $\omega_{g, n r} \sim$ $k H z$, respectivamente, ou seja, da ordem de $10^{-3}$ se comparada com a frequência de plasmas, $\omega_{p e} \sim \mathrm{MHz}$, de forma que para o estudo dos GAM e ZF podemos considerar o regime eletrostático, $\partial \boldsymbol{E} / \partial t \sim 0$.

Considera-se também a condição de quasineutralidade do plasma em decorrência de a frequência dos ZF e dos GAM ser bem menor do que a frequência de plasmas, $\omega_{p e}$, de forma que os elétrons são capazes de neutralizar rapidamente qualquer densidade de carga que possa existir. Isto nos permite desprezar o termo $\tau$ nas equações 3.1 e 3.9. A validade desta aproximação se limita a regiões cujos comprimentos característicos sejam maiores do que o comprimento de Debye, $\lambda_{D}[17]$.

Pelo fato de ZF e GAM ocorrerem, principalmente, na borda da coluna de plasma, ou seja, em regiões de baixa pressão hidrostática e consequentemente de baixo valor de $\beta$, podemos desprezar perturbações temporais do campo magnético, ou seja, $\frac{\partial B}{\partial t} \sim 0$, conforme mostramos na seção 3.2. Desta forma, considera-se a aproximação eletrostática em tais regiões. Nesta aproximação, de acordo com a lei de Faraday, 3.3, e a identidade vetorial A.6, podemos escrever o campo elétrico como o gradiente de uma função escalar, $\Phi$, conhecida como potencial eletrostático.

Se considerarmos as aproximações feitas anteriormente para um plasma perfeitamente condutor, isto é, $\eta=0$, o conjunto de equações a serem resolvidas é:

$$
\begin{gathered}
\nabla \cdot \boldsymbol{B}=0, \\
\boldsymbol{J} \approx \frac{1}{\mu_{0}} \nabla \times \boldsymbol{B}, \quad \nabla \cdot \boldsymbol{J}=0, \\
\rho \frac{D \boldsymbol{v}}{D t}+\nabla p-\boldsymbol{J} \times \boldsymbol{B}=0,
\end{gathered}
$$




$$
\begin{gathered}
\frac{\partial \rho}{\partial t}+\nabla \cdot(\rho \boldsymbol{v})=0, \\
\boldsymbol{E}+\boldsymbol{v} \times \boldsymbol{B}=0, \quad \boldsymbol{E}=-\boldsymbol{\nabla} \Phi \quad \mathrm{e} \\
\frac{D p}{D t}+\gamma p \boldsymbol{\nabla} \cdot \boldsymbol{v}=0,
\end{gathered}
$$

É comum dizer, ao considerar plasmas sem resistividade (plasmas perfeitamente condutores), que as linhas de campo magnético estão congeladas no plasma, ou seja, o plasma se move junto com elas [10].

\subsection{Equações da MHD ideal perturbadas em primeira ordem}

Para determinar a relação de dispersão, $\omega(\boldsymbol{k})$, para modos que surgem no plasma, como por exemplo os GAM e ZF, objetos de estudo deste trabalho, é conveniente utilizar a teoria de perturbações até primeira ordem e, consequentemente, linearizar as equações da MHD, 3.12-3.17. Nesta teoria consideramos que as grandezas físicas relevantes para o problema são da seguinte forma:

$$
\xi(\boldsymbol{r}, t)=\xi_{0}(\boldsymbol{r})+\xi_{1}(\boldsymbol{r}, t) \quad \text { onde } \quad \xi_{1}(\boldsymbol{r}, t)=\xi_{1}(\boldsymbol{r}) \mathrm{e}^{-i \omega t}, \quad \text { com } \quad \frac{\left|\xi_{1}\right|}{\left|\xi_{0}\right|} \ll 1 .
$$

Considera-se que o plasma se encontra em equilíbrio mecânico estável até que algum processo físico perturbe este equilíbrio produzindo oscilações temporais de frequência $\omega$. Nesta situação a grandeza física $\xi$, que antes era dada por $\xi=\xi_{0}$, passa a ser $\xi=\xi_{0}+\xi_{1}$.

Antes de derivar a relação de dispersão para os modos do plasma, é necessário determinar as grandezas físicas quando o plasma se encontra em equilíbrio. Neste caso, não há variação temporal das grandezas físicas, de forma que as equações 3.12 - 3.17 podem ser escritas como 


$$
\begin{gathered}
\boldsymbol{\nabla} \cdot \boldsymbol{B}=0, \\
\rho_{0}\left(\boldsymbol{v}_{\mathbf{0}} \cdot \boldsymbol{\nabla}\right) \boldsymbol{v}_{\mathbf{0}}+\nabla p_{0}+\frac{1}{\mu_{0}} \boldsymbol{B} \times(\boldsymbol{\nabla} \times \boldsymbol{B})=0, \\
\boldsymbol{\nabla} \cdot\left(\rho_{0} \boldsymbol{v}_{\mathbf{0}}\right)=0, \\
-\boldsymbol{\nabla} \Phi_{0}+\boldsymbol{v}_{\mathbf{0}} \times \boldsymbol{B}=0 \quad \mathrm{e} \\
\left(\boldsymbol{v}_{\mathbf{0}} \cdot \boldsymbol{\nabla}\right) p_{0}+\gamma p_{0}\left(\boldsymbol{\nabla} \cdot \boldsymbol{v}_{\mathbf{0}}\right)=0 .
\end{gathered}
$$

O problema de encontrar as grandezas físicas no equilíbrio em tokamaks com rotação é incontestavelmente um problema não resolvido até hoje, pelo menos analiticamente. Neste trabalho propomos modelos apropriados para descrever o equilíbrio em três casos particulares: tokamaks sem rotação (ENR), em que $\boldsymbol{v}_{\mathbf{0}}=0$, tokamaks com rotação toroidal (ETRF), em que $\boldsymbol{v}_{\mathbf{0}}=v_{0} \hat{e}_{\phi}$ e equilíbrio com rotação binormal (EBRF), em que a velocidade de equilíbrio é praticamente poloidal, $\boldsymbol{v}_{\mathbf{0}} \approx v_{0} \hat{e}_{\theta}$. O terceiro caso trata-se de um trabalho novo no qual consideramos o potencial eletrostático conhecido e, juntamento com o campo magnético, a causa da rotação binormal no plasma, que ocorre devido ao movimento de deriva, $\boldsymbol{E} \times \boldsymbol{B}$. No entanto, ressalvamos que, no equilíbrio com rotação, o equilíbrio não será descrito de forma auto-consistente.

Para tokamaks de alta razão de aspecto, apenas as perturbações de primeira ordem desempenham um papel importante na determinação dos modos de oscilação. Os termos de ordem superior são importantes somente na saturação de modos instáveis. Entretanto mecanismos de saturação não serão tratados neste trabalho. As equações $3.12-3.17$ perturbadas até primeira ordem são

$$
\nabla \cdot J_{1}=0
$$




$$
\begin{gathered}
-i \omega \rho_{0} \boldsymbol{v}_{\mathbf{1}}+\rho_{0}\left[\left(\boldsymbol{v}_{\mathbf{0}} \cdot \boldsymbol{\nabla}\right) \boldsymbol{v}_{\mathbf{1}}+\left(\boldsymbol{v}_{\mathbf{1}} \cdot \boldsymbol{\nabla}\right) \boldsymbol{v}_{\mathbf{0}}\right]+\rho_{1}\left(\boldsymbol{v}_{\mathbf{0}} \cdot \boldsymbol{\nabla}\right) \boldsymbol{v}_{\mathbf{0}}+\boldsymbol{\nabla} p_{1}-\boldsymbol{J}_{\mathbf{1}} \times \boldsymbol{B}=0 \\
-i \omega \rho_{1}+\left[\left(\boldsymbol{v}_{\mathbf{0}} \cdot \boldsymbol{\nabla}\right) \rho_{1}+\left(\boldsymbol{\nabla} \cdot \boldsymbol{v}_{\mathbf{0}}\right) \rho_{1}\right]+\left[\left(\boldsymbol{v}_{\mathbf{1}} \cdot \boldsymbol{\nabla}\right) \rho_{0}+\left(\boldsymbol{\nabla} \cdot \boldsymbol{v}_{\mathbf{1}}\right) \rho_{0}\right]=0 \\
-\boldsymbol{\nabla} \Phi_{1}+\boldsymbol{v}_{\mathbf{1}} \times \boldsymbol{B}=0 \quad \mathrm{e} \\
-i \omega p_{1}+\left(\boldsymbol{v}_{\mathbf{0}} \cdot \boldsymbol{\nabla}\right) p_{1}+\left(\boldsymbol{v}_{\mathbf{1}} \cdot \boldsymbol{\nabla}\right) p_{0}+\gamma p_{1}\left(\boldsymbol{\nabla} \cdot \boldsymbol{v}_{\mathbf{0}}\right)+\gamma p_{0}\left(\boldsymbol{\nabla} \cdot \boldsymbol{v}_{\mathbf{1}}\right)=0
\end{gathered}
$$

em que desprezamos perturbações do campo magnético de acordo com a aproximação eletrostática. Esta aproximação pode ser justificada ao comparar a ordem de grandeza dos dois últimos termos da equação 3.14 considerando perturbações do campo magnético $\left(\boldsymbol{B}_{1}\right)$. Sendo assim temos

$$
\nabla p_{1} \sim \boldsymbol{J}_{1} \boldsymbol{B}_{0}+\boldsymbol{J}_{0} \boldsymbol{B}_{1}
$$

Considerando a aproximação de quasineutralidade ${ }^{1}$ do plasma e que não haja perturbação na temperatura do plasma ${ }^{2}\left(T_{0}\right)$, ao tratar o plasma como um gás perfeito, podemos escrever a pressão perturbada como

$$
p_{1} \approx n_{1} T_{0}=\frac{n_{1}}{n_{0}} n_{0} T_{0}=\frac{n_{1}}{n_{0}} p_{0}
$$

de forma que a relação entre as ordens de grandeza de cada termo de 3.29,

$$
[L]^{-1} \frac{n_{1}}{n_{0}} p_{0} \sim \mu_{0}^{-1}[L]^{-1} B_{0} B_{1} \Longrightarrow \frac{B_{1} / B_{0}}{n_{1} / n_{0}} \sim \frac{\mu_{0} p_{0}}{B_{0}^{2}} \sim \beta \ll 1
$$

\footnotetext{
${ }^{1}$ Esta aproximação nos permite escrever $n_{1 i} \sim n_{1 e} \sim n_{1}$, onde $n_{1 i}$ e $n_{1 e}$ são a densidade de íons e de elétrons perturbadas, respectivamente.

${ }^{2} T_{0}$ é a soma da temperatura dos íons, $T_{0 i}$, e dos elétrons, $T_{0 e}$.
} 
justifica a aproximação eletrostática para regimes de baixa pressão e consequentemente baixos valores de $\beta$.

No capítulo 4, descrevemos o plasma no equilíbrio em tokamaks de secção circular considerando os três casos mencionados anteriormente: ENR, ETRF e EBRF. 


\section{Capítulo 4}

\section{Equilíbrio MHD}

Neste capítulo, apresentamos uma discussão sobre sistemas cilíndricos e tokamaks de secção circular. Também resolvemos as equações da MHD ideal (equações 3.19 -3.23 do capítulo 3) no equilíbrio. Embora tratemos apenas tokamaks de secção circular, discussões sobre o problema para tokamaks de secção não-circular também são apresentadas. Pelo fato de estarmos lidando apenas com grandezas no equilíbrio neste capítulo, suprimimos o índice 0 de tais grandezas físicas, embora elas devam ficar subentendidas. Utilizamos, porém, o índice 0, neste capítulo, para as grandezas físicas do problema em uma dimensão, ou seja, em sistemas cilíndricos. Isto ficará claro mais adiante.

O objetivo da teoria do equilíbrio MHD é descobrir geometrias magnéticas capazes de confinar e isolar o plasma quente das paredes materiais e permitir estabilidade macroscópica a valores suficientemente altos de $\beta$. Entretanto, neste trabalho, conforme mencionado anteriormente estamos considerando apenas regimes de baixos valores de $\beta$, o que, mesmo assim, não invalida o modelo da MHD.

As equações da MHD ideal no equilíbrio são

$$
\begin{gathered}
\boldsymbol{\nabla} \cdot \boldsymbol{B}=0, \\
\rho(\boldsymbol{v} \cdot \boldsymbol{\nabla}) \boldsymbol{v}+\nabla p-\boldsymbol{J} \times \boldsymbol{B}=0, \\
\boldsymbol{J}=\frac{1}{\mu_{0}} \nabla \times \boldsymbol{B},
\end{gathered}
$$




$$
\begin{gathered}
\boldsymbol{\nabla} \cdot(\rho \boldsymbol{v})=0, \\
-\boldsymbol{\nabla} \Phi+\boldsymbol{v} \times \boldsymbol{B}=0 \quad \mathrm{e} \\
(\boldsymbol{v} \cdot \boldsymbol{\nabla}) p+\gamma p(\boldsymbol{\nabla} \cdot \boldsymbol{v})=0 .
\end{gathered}
$$

Para resolver este sistema, solúvel em princípio, consideramos que há simetria axial, ou simetria toroidal, para tokamaks. Tal simetria, em coordenadas cilíndricas globais, $(R, \varphi, Z)$, e coordenadas pseudo-toroidais, $(r, \theta, \phi)$, corresponde ao ângulo $\phi=-\varphi$, de forma que $\partial_{\phi} \xi=0$, onde $\xi$ representa qualquer grandeza física escalar ${ }^{1}$. Este tipo de equilíbrio, com esta simetria, é conhecido como equilíbrio em duas dimensões, pois a grandeza genéria $\xi$ será da forma $\xi=\xi(R, Z)$, em coordenadas cilíndricas globais, ou $\xi=\xi(r, \theta)$, em coordenadas pseudo-toroidais.

É comum também, em modelos analíticos teóricos para tokamaks de seç̧ão circular, resolver o problema em uma dimensão ${ }^{2}$. Torna-se conveniente, então, utilizar coordenadas cilíndricas locais, $(r, \theta, z)$. Neste caso há simetria também com relação à coordenada poloidal, $\theta$, e a simetria axial corresponde à simetria em $z$, onde vale a relação: $d z=R_{0} d \varphi$. Desta forma, considera-se que $\xi=\xi(r)^{3}$.

Quando se trata de tokamaks, a simetria com relação ao ângulo $\theta$ é uma aproximação local em que se considera $\varepsilon \rightarrow 0$; entretanto, mesmo quando considerarmos apenas simetria toroidal, utilizaremos a aproximação de alta razão de aspecto, ou seja, $\varepsilon \ll 1$, de forma que termos de ordem $O\left(\varepsilon^{2}\right)$ são desprezados.

A quebra de simetria toroidal ocorre em tokamaks reais como uma consequência do espaçamento entre as bobinas toroidais, entre outros fatores. Esta falta de simetria é ressaltada na borda da coluna de plasma em tokamaks reais. Entretanto, neste trabalho, consideramos que há simetria toroidal, pois o intuito é focar em outros fenômenos físicos importantes.

\footnotetext{
${ }^{1}$ Neste trabalho $\xi$ poderá ser a pressão, a densidade, a função de fluxo, o potencial elétrico, componentes da velocidade ou componentes do campo magnético

${ }^{2}$ Menos aproximações são necessárias para resolver o problema analiticamente em uma dimensão e alguns conceitos físicos são ressaltados.

${ }^{3}$ Caso se trate de um tokamak de secção não-circular $\xi=\xi(r, \theta)$.
} 


\subsection{Equilíbrio em uma dimensão}

O equilíbrio em uma dimensão para um tokamak de secção circular é ilustrado na figura 4.1, a qual descreve a ultilização de coordenadas cilíndricas locais para expressar as grandezas físicas relevantes para o problema.

Conforme dissemos anteriormente, utilizamos o índice 0 para expressar as grandezas do equilíbrio em uma dimensão. A equação 4.1 nos permite expressar o campo magnético como

$$
\boldsymbol{B}_{0}=\nabla \Psi_{0} \times \hat{e}_{z}+B_{z} \hat{e}_{z}
$$

onde $\Psi_{0}=\Psi_{0}(r, \theta)$ é uma função arbitrária conhecida como função de fluxo. Em sistemas cilíndricos de secção circular, $\Psi_{0}=\Psi_{0}(r)$.

Podemos mostrar que a equação 4.7 é uma solução válida para o campo magnético calculando $\boldsymbol{\nabla} \cdot \boldsymbol{B}_{0}$. Tal demonstração é feita no apêndice C.

Da equação 4.3 obtemos a densidade de corrente $\boldsymbol{J}_{\mathbf{0}}=J_{z} \hat{e}_{z}+J_{\perp} \hat{\boldsymbol{e}}_{\perp}$, cujas componentes são dadas por

$$
J_{z}=-\frac{1}{\mu_{0}} \nabla^{2} \Psi_{0} \quad \text { e } \quad J_{\perp} \hat{\boldsymbol{e}}_{\perp}=-\frac{1}{\mu_{0}} \hat{e}_{z} \times \nabla B_{z}
$$

sendo $\hat{\boldsymbol{e}}_{\perp}$ um versor perpendicular aos versores $\hat{\boldsymbol{e}}_{\Psi_{0}}$ e $\hat{e}_{z}$, onde $\hat{\boldsymbol{e}}_{\Psi_{0}}$ é o versor normal às superfícies magnéticas, definido por

$$
\hat{e}_{\Psi_{0}}=\frac{\nabla \Psi_{0}}{\left\|\nabla \Psi_{0}\right\|}
$$

Se considerarmos plasmas sem rotação, ou equilíbrio estático, isto é, $\boldsymbol{v}_{\mathbf{0}}=0$, e tomarmos a componente paralela ao campo magnético,

$$
\hat{\boldsymbol{e}}_{\|}=\frac{\boldsymbol{B}_{0}}{\left\|\boldsymbol{B}_{0}\right\|}
$$

da equação do balanço de momento, 4.2, obtemos o Jacobiano $\jmath\left(\Psi_{0}, p_{0}\right)$, 


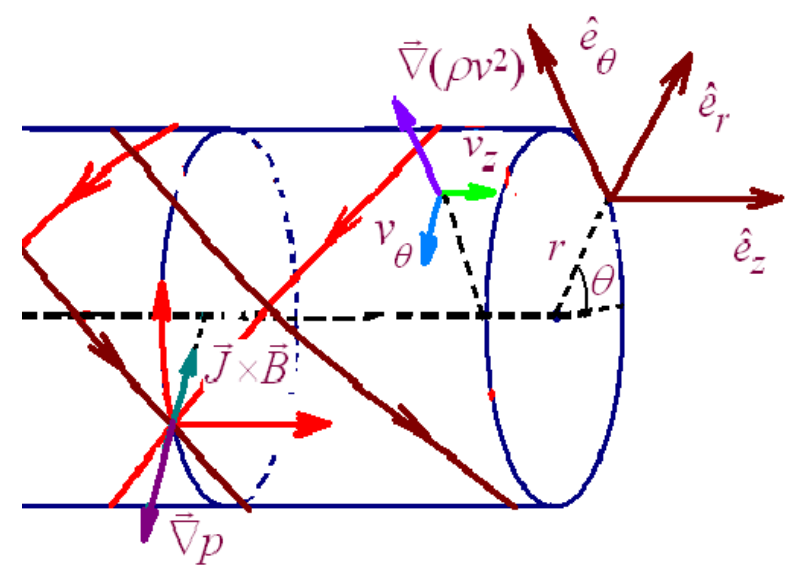

Figura 4.1: Equilíbrio em uma dimensão

$$
\nabla p_{0} \cdot \nabla \Psi_{0}=\frac{1}{r}\left(\frac{\partial p_{0}}{\partial r} \frac{\partial \Psi_{0}}{\partial \theta}-\frac{\partial p_{0}}{\partial \theta} \frac{\partial \Psi_{0}}{\partial r}\right)=\jmath\left(\Psi_{0}, p_{0}\right)=0
$$

Analogamente, a componente paralela à $\boldsymbol{J}_{\mathbf{0}}$ de 4.2 nos fornece

$$
\hat{e}_{z} \cdot\left(\nabla p_{0} \times \nabla B_{z}\right)=\jmath\left(B_{z}, p_{0}\right)=0 .
$$

Pelo fato de os jacobianos das equações 4.11 e 4.12 se anularem, concluímos que $p_{0}=$ $p_{0}\left(\Psi_{0}\right)$ e $B_{z}=B_{z}\left(\Psi_{0}\right)$. As passagens matemáticas para obter as equações 4.11 e 4.12 são feitas em detalhe no apêndice $\mathrm{C}$.

Ao multiplicar 4.2 por $\hat{\boldsymbol{e}}_{\Psi_{0}}$, obtemos

$$
\frac{d}{d \Psi_{0}}\left(p_{0}+\frac{B_{z}^{2}}{2 \mu_{0}}\right)=-\frac{1}{\mu_{0}} \nabla^{2} \Psi_{0}=J_{z}
$$

que, para tokamaks de secção circular, pode ser escrita como

$$
\frac{d}{d r}\left(p_{0}+\frac{B_{\theta 0}^{2}+B_{z}^{2}}{2 \mu_{0}}\right)=-\frac{B_{\theta 0}^{2}}{\mu_{0} r}
$$

onde utilizamos o desenvolvimento da equação 4.7, 


$$
\frac{1}{R_{0}} \frac{d \Psi_{0}}{d r}=B_{\theta 0}(r), \quad \boldsymbol{B}_{0}=B_{\theta 0} \hat{e}_{\theta}+B_{z} \hat{e}_{z}
$$

e a regra da cadeia,

$$
\frac{d}{d \Psi_{0}}=\left(\frac{d \Psi_{0}}{d r}\right)^{-1} \frac{d}{d r}=\frac{1}{B_{\theta 0}} \frac{d}{d r}
$$

A equação 4.14 descreve o balanço entre a pressão hidrostática e a pressão magnética em sistemas cilíndricos de secção circular.

Em plasmas com rotação, 4.14 deve ser substituída por

$$
\frac{d}{d r}\left(p_{0 t}+\frac{B_{\theta 0}^{2}+B_{z}^{2}}{2 \mu_{0}}\right)=-\frac{B_{\theta 0}^{2}}{\mu_{0} r}+\rho \frac{v_{\theta 0}^{2}}{r}
$$

onde a velocidade de equilíbrio é $\boldsymbol{v}_{0}=v_{\theta 0}(r) \hat{e}_{\theta}+v_{z}(r) \hat{e}_{z}$, e teríamos que considerar o campo elétrico que surge no referencial do laboratório

$$
\boldsymbol{E}_{\mathbf{0}}=\boldsymbol{E}_{\mathbf{0}}(r)=\left(v_{z} B_{\theta 0}-v_{\theta 0} B_{z}\right) \hat{e}_{r}
$$

Supondo que a velocidade do plasma não altere o campo magnético, poderíamos separar a equação 4.17 em duas, considerando que a pressão seja $p_{0 t}=p_{0}+p_{0 c}$, onde $p_{0 t}$ representa a pressão cinética total, $p_{0}$ é a pressão cinética na ausência de rotação e $p_{0 c}$ é a contribuição para a pressão por parte da rotação poloidal de equilíbrio, ou seja,

$$
\frac{d}{d r}\left(p_{0}+\frac{B_{\theta 0}^{2}+B_{z}^{2}}{2 \mu_{0}}\right)=-\frac{B_{\theta 0}^{2}}{\mu_{0} r} \quad \text { e } \quad \frac{d p_{0 c}}{d r}=\rho \frac{v_{\theta 0}^{2}}{r}
$$

Para este modelo unidimensional, para o caso sem rotação, nota-se que duas grandezas físicas são determinadas de forma arbitrária, de acordo com a equação 4.14. Esta é uma das maiores falhas do modelo da MHD ideal. Para o caso com rotação, conforme mostra as equações 4.17 e 4.18, a arbitrariedade é ainda maior: cinco grandezas são determinada de forma arbitrária. Se consideramos que o campo magnético não se altera com a rotação do plasma, a arbitrariedade pode ser reduzida a quatro incógnitas, conforme mostra 4.18 e 4.19 .

A equação 4.19 mostra que a rotação poloidal, independente do sentido, faz com que 
a pressão hidrostática do plasma aumente, consequentemente aumentando o valor de $\beta_{0}$, uma vez que a pressão magnética permanece inalterada. Este efeito faz com que o plasma tenha uma tendência a se deslocar para a borda.

\subsection{Equilíbrio em duas dimensões}

Analogamente à 4.7, conforme mostramos no apêndice C, podemos expressar o campo magnético de tokamaks como

$$
\boldsymbol{B}=\frac{1}{R}\left(\nabla \Psi \times \hat{e}_{\phi}+F \hat{e}_{\phi}\right)
$$

A função $\Psi=\Psi(r, \theta)=\Psi(R, Z)$ está relacionada com o fluxo magnético poloidal, o qual é dado por

$$
\Psi_{\theta}=\int_{S} \boldsymbol{B}_{\boldsymbol{\theta}} \cdot d \boldsymbol{S}
$$

enquanto a função $F=F(r, \theta)=F(R, Z)=R B_{\phi}$ está relacionada com a corrente poloidal do plasma e das bobinas toroidais.

As componentes $B_{R}$ e $B_{Z}$ do campo magnético, em coordenadas cilíndricas globais, podem ser expressas em função de $\Psi$ como

$$
B_{R}=-\frac{1}{R} \frac{\partial \Psi}{\partial Z} \quad \text { e } \quad B_{Z}=\frac{1}{R} \frac{\partial \Psi}{\partial R}
$$

A conveniente escolha de um elemento de área na posição $Z=0$, em que, neste plano, $B_{\theta}=B_{Z}$, conforme mostra a figura 4.2 , nos permite obter a relação

$$
\Psi=\frac{\Psi_{\theta}}{2 \pi}
$$

a partir da substituição de 4.22 em 4.21 .

A relação entre $\Psi$ e a corrente de plasma $I_{P}=\int_{A} \boldsymbol{J}_{\phi} \cdot \boldsymbol{d} \boldsymbol{A}$ é obtida substituindo-se 4.20 na lei de Ampère, 4.3, 


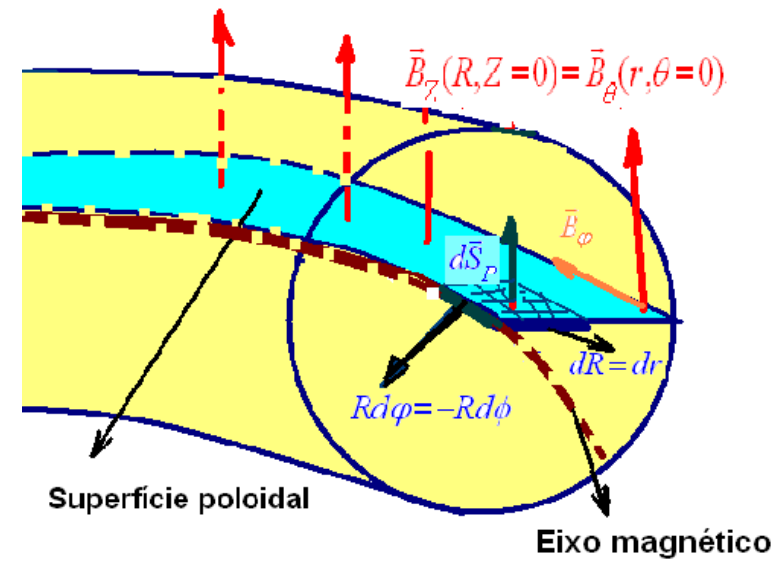

Figura 4.2: Na superfície do plasma $Z=0, B_{\theta}=B_{Z}$, de forma que é conveniente escolher esta superfície para calcular o fluxo magnético poloidal.

$$
\boldsymbol{J}=\frac{1}{\mu_{0}} \nabla \times \boldsymbol{B}
$$

de forma que as seguintes relações podem ser obtidas a partir destas equações;

$$
\Delta^{*} \Psi \hat{e}_{\phi}=-\mu_{0} R \boldsymbol{J}_{\phi}, \quad \nabla F \times \hat{e}_{\phi}=\mu_{0} R\left(\boldsymbol{J}_{\boldsymbol{r}}+\boldsymbol{J}_{\boldsymbol{\theta}}\right) \quad \text { e } \quad I_{P}=-\frac{1}{\mu_{0}} \int_{A} \frac{\Delta^{*} \Psi}{R} \hat{e}_{\phi} \cdot \boldsymbol{d} \boldsymbol{A}
$$

onde

$$
\Delta^{*}=\frac{\partial^{2}}{\partial R^{2}}-\frac{1}{R} \frac{\partial}{\partial R}+\frac{\partial^{2}}{\partial Z^{2}}
$$

Em 4.25, $I_{T}$ é conhecida mas $\boldsymbol{J}_{\boldsymbol{\theta}}$ é arbitrariamente determinada neste modelo. A lei circuital de Ampère (A.15 aplicado à 4.3), para um tokamak de secção circular, permite obter $F$ na região do vácuo (fora da coluna de plasma),

$$
F_{v}=-\frac{\mu_{0} N}{2 \pi} I_{T}
$$

onde $N$ é o número de bobinas toroidais.

Assim como para sistemas cilíndricos, também descrevemos para sistemas toroidais os casos de equilíbrio sem rotação (ENR), $\boldsymbol{v}=0$, e de equilíbrio com rotação (ER), $\boldsymbol{v} \neq 0$. Em ambos os casos, por se tratar de sistemas toroidais, há uma quebra de 
simetria com relação à coordenada poloidal. Esta quebra de simetria torna o problema consideravelmente mais complexo, de forma que, mesmo para ENR, temos que considerar a aproximação de alta razão de aspecto, $A=R_{0} / a \sim 1 / \varepsilon=R_{0} / r \gg 1$, para poder resolver o problema analiticamente. O problema de ER só foi resolvido analiticamente para alguns casos bastantes particulares [27].

Nas subseções que se seguem resolvemos primeiramente o equilíbrio sem rotação ENR considerando a aproximação de alta razão de aspecto, ou seja, $\varepsilon=r / R_{0} \ll 1$, de acordo com [28] e, em seguida, analisamos o equilíbrio com rotação - ER para resolver dois casos particulares: rotação toroidal - ETRF e rotação binormal - EBRF; sendo que o último acreditamos se tratar de um resultado novo.

\subsubsection{ENR e a equação de Grad-Shafranov}

Para resolver o caso de ENR, as equações a serem resolvidas (4.1-4.5) se reduzem a

$$
\boldsymbol{\nabla} p_{0}=\boldsymbol{J}_{\mathbf{0}} \times \boldsymbol{B}=0 \quad \text { e } \quad \boldsymbol{\nabla} \Phi_{0}=0
$$

em que $\boldsymbol{B}=\left(\boldsymbol{\nabla} \Psi \times \hat{e}_{\phi}+F \hat{e}_{\phi}\right) / R$, de acordo com 4.20.

De acordo com 4.28, $\Phi_{0}=0$, uma vez que o potencial elétrico é definido a menos de uma constante, a qual podemos defini-la $\left(\Phi_{0}=0\right)$ por conveniência. O importante é notar que não há campo elétrico de equilíbrio, o que, conforme mostramos mais adiante, não ocorre na presença de rotação.

Ao multiplicarmos 4.28 escalarmente por $\boldsymbol{B}$ e por $\boldsymbol{J}$, analogamente ao que foi feito para o equilíbrio em uma dimensão (equações 4.11 e 4.12), é possível mostrar que $p=$ $p(\Psi)$ e $F=F(\Psi)$. Os detalhes desta demonstração são apresentados no apêndice C.

Ao tomarmos a componente $\hat{\boldsymbol{e}}_{\Psi}=\nabla \Psi /|\nabla \Psi|$ de 4.28 , utilizando também 4.25 se obtém a equação de Grad-Shafranov,

$$
\Delta^{*} \Psi=-\mu_{0} R^{2} \frac{d p}{d \Psi}-\frac{1}{2} \frac{d F^{2}}{d \Psi}
$$

que possibilita determinar determinar $\Psi$ e consequentemente $B_{\theta}$ e $B_{r}$ (em tokamaks de secção não circular).

No apêndice C, resolvemos a equação de Grad-Shafranov para tokamaks de secção circular. A solução encontrada é aproximada e é válida apenas para tokamaks de alta razão de aspecto. Tal equação foi resolvida substituindo a solução tentativa, 


$$
\Psi=\Psi_{0}(r)+\epsilon \Psi_{1}(r, \theta)
$$

onde

$$
\epsilon=\frac{a}{R_{0}}
$$

em 4.29 e considerando o desenvolvimento em potências de $\epsilon$, em que $\epsilon \ll 1$, de acordo com a metodologia descrita por [28]. A solução encontrada foi

$$
\Psi(r, \theta)=\Psi_{0}(r)+\frac{d \Psi_{0}}{d r} \Delta_{S}(r) \cos \theta
$$

onde $\Delta_{S}(r)$, que é conhecido como deslocamento de Shafranov, é dado por

$$
\Delta_{S}(r)=\frac{1}{R_{0}} \int_{r}^{a} d r^{\prime} \frac{1}{r^{\prime} B_{\theta 0}^{2}} \int_{0}^{r^{\prime}} d r^{\prime \prime}\left(2 \mu_{0} r^{\prime \prime} \frac{d p}{d r^{\prime \prime}}-r^{\prime \prime} B_{\theta 0}^{2}\right)
$$

Nota-se que no limite $r \rightarrow a, \Delta_{S}(r \rightarrow a)=0$, de forma que, quanto mais externas forem as superfícies magnéticas, menor é a influência do deslocamento de shafranov.

Se considerarmos apenas algumas superfícies magnéticas, localizadas próximas à fronteira plasma-vácuo e próximas umas das outras, com base no argumento acima, e ao redefinir $R_{0}$, da forma $R_{0} \rightarrow R_{0}+\Delta_{S}\left(r=r_{0}\right)$, onde $r_{0}$ é um raio específico próximo de $a$, podemos utilizar a aproximação,

$$
\Psi \approx \Psi_{0}(r)
$$

na qual fica subentendido que $r \approx r_{0}$. Tal aproximação é conhecida como aproximação local e é a base para se construir um modelo simplificado para estudar analiticamente os ZF e GAM localmente, isto é, localizados na borda da coluna de plasma. Ao longo deste trabalho consideramos esta aproximação.

Sendo assim, o campo magnético para tokamak de secção circular é dado por 4.20, ou seja,

$$
\boldsymbol{B}=\boldsymbol{B}(r, \theta)=\frac{1}{R} \frac{d \Psi_{0}}{d r} \hat{e}_{\theta}+B_{\phi}(r, \theta) \hat{e}_{\phi},
$$


em que

$$
B_{\theta 0}(r)=\frac{1}{R_{0}} \frac{d \Psi_{0}}{d r} \quad \text { e } \quad R_{0} B_{\phi 0}(r)=R B_{\phi}(r, \theta)=F\left(\Psi_{0}\right)
$$

Desta forma fica determinado o campo magnético para um tokamak de secção circular em termos do campo magnético de um sistema cilíndrico,

$$
\boldsymbol{B}=\frac{R_{0}}{R}\left(\frac{\varepsilon}{q} \hat{e}_{\theta}+\hat{e}_{\phi}\right) B_{\phi 0}(r)
$$

onde utilizamos

$$
B_{\theta 0}(r)=f(r) B_{\phi 0}(r) \Longrightarrow f(r)=\frac{B_{\theta 0}(r)}{B_{\phi 0}(r)}=\frac{r / R_{0}}{\frac{r B_{\phi 0} R_{0} / R}{R_{0} B_{\theta 0} R_{0} / R}}=\frac{\varepsilon}{q} .
$$

No limite $\varepsilon \rightarrow 0$, voltamos ao problema unidimensional, pois utilizando 4.20 podemos escrever 4.28 como

$$
\frac{d}{d r}\left(p+\frac{B_{\theta 0}^{2}+B_{\phi 0}^{2}}{2 \mu_{0}}\right)=-\frac{B_{\theta 0}^{2}}{r}
$$

em que neste limite $B_{\phi 0} \rightarrow B_{z}$, e portanto 4.38 se reduz a 4.14 .

O interesse em resolver o problema bidimensional, para tokamaks de secção circular, é obter a dependência em $\theta$ do campo magnético, a qual surge como consequência da curvatura geodésica do tokamak. Esta curvatura geodésica é a responsável pelo surgimento de ZF e GAM no plasma, conforme é mostrado no capítulo 5.

Como utilizamos a aproximação $\Psi \approx \Psi_{0}(r)$ e vimos anteriormente que $p=p(\Psi) \approx$ $p\left(\Psi_{0}\right)$ então consideramos $p=p(r)$ e $\rho=\rho(r)$ no ENR, em que a última condição é obtida considerando o plasma como um gás perfeito no equilíbrio,

$$
p=n_{i} T_{i}+n_{e} T_{e} \approx 2 n T
$$

onde $n_{i} \approx n_{e}=n$ é a densidade de íons ${ }^{4}$ e $n_{e}$ é a densidade de elétrons e consideramos

\footnotetext{
${ }^{4}$ Íons por unidade de volume
} 


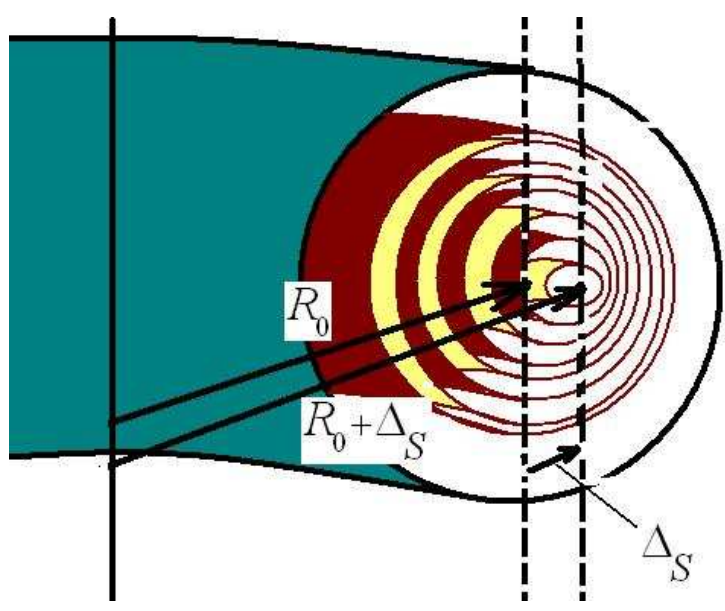

Figura 4.3: Deslocamento de Shafranov $\left(\Delta_{S}\right)$ : Secção transversal de um tokamak de secção circular no qual são mostradas as superfícies magnéticas.

que a temperatura dos íons e dos elétrons são próximas, $T_{i} \approx T_{e}=T(r)^{5}$.

\subsubsection{Equilíbrio com rotação (ER)}

Para o estudo de rotações no equilíbrio, partimos das equações 4.1-4.6. Inicialmente combinamos 4.2 e 4.3 e utilizamos a identidade vetoriai A.12 para escrever a equação do balanço de momento como

$$
\boldsymbol{\nabla}\left(p+\frac{B^{2}}{2 \mu_{0}}\right)=\frac{1}{\mu_{0}}(\boldsymbol{B} \cdot \boldsymbol{\nabla}) \boldsymbol{B}-\rho(\boldsymbol{v} \cdot \boldsymbol{\nabla}) \boldsymbol{v}
$$

A seguir desenvolvemos a equação da continuidade

$$
(\boldsymbol{\nabla} \cdot \boldsymbol{v}) \rho+(\boldsymbol{v} \cdot \boldsymbol{\nabla}) \rho=0
$$

e expressamos a conservação da entropia como a evolução da pressão,

$$
\gamma(\boldsymbol{\nabla} \cdot \boldsymbol{v}) p+(\boldsymbol{v} \cdot \boldsymbol{\nabla}) p=0
$$

Ao invés de resolver este sistema de equações no sistema de coordenadas pseudo-

\footnotetext{
${ }^{5}$ Consideramos que qualquer desequilíbrio na temperatura ao longo de uma superfície magnética é instantaneamente estabilizado, ou seja, a condutividade térmica poloidal e toroidal é considerada infinita, dai segue $T=T(\Psi) \approx T(r)$.
} 
toroidais, utilizamos um outro sistema, o qual está relacionado com a curvatura geodésica do campo magnético, com as superfícies magnéticas e com o movimento de deriva. Tal escolha é conveniente, não apenas neste capítulo, mas também e, principalmente, nos próximos capítulos.

Os versores, já mencionados anteriormente, deste sistema de coordenada são definidos por

$$
\hat{\boldsymbol{e}}_{\Psi}=\frac{\nabla \Psi}{\|\nabla \Psi\|}, \quad \hat{\boldsymbol{e}}_{\|}=\frac{\boldsymbol{B}}{B} \quad \text { e } \quad \hat{\boldsymbol{e}}_{\perp}=\hat{\boldsymbol{e}}_{\Psi} \times \hat{\boldsymbol{e}}_{\|}
$$

de forma que, como $\boldsymbol{E}=\frac{d \Phi}{d \Psi} \hat{\boldsymbol{e}}_{\Psi}$, podemos utilizar a lei de Ohm, 4.5, para expressar a velocidade como

$$
\boldsymbol{v}=\omega_{0} R \hat{\boldsymbol{e}}_{\perp}+u_{0} R_{0} \hat{\boldsymbol{e}}_{\|}
$$

onde

$$
\omega_{0} \approx-\frac{1}{R B_{\phi}} \frac{d \Phi}{d \Psi}
$$

está associada à velocidade de deriva e

$$
u_{0}=\frac{\boldsymbol{v} \cdot \hat{\boldsymbol{e}}_{\|}}{R_{0}}
$$

representa a componente da velocidade paralela ao campo magnético.

Ao considerar $\varepsilon \ll 1$ e aproximar localmente $\left(\hat{\boldsymbol{e}}_{\Psi} \approx \hat{e}_{r}\right)$, podemos definir os seguintes operadores

$$
\nabla_{\|}=\hat{\boldsymbol{e}}_{\|} \cdot \boldsymbol{\nabla}=\frac{1}{q R_{0}} \frac{\partial}{\partial \theta} e \nabla_{\perp}=\hat{\boldsymbol{e}}_{\perp} \cdot \boldsymbol{\nabla}=-\frac{1}{\varepsilon q} \frac{\partial}{\partial \theta}
$$

de forma que as equações 4.42 e 4.43 são dadas por

$$
\rho=D_{\theta} \rho \quad \mathrm{e}
$$




$$
p=\frac{1}{\gamma} D_{\theta} p
$$

onde o operador $D_{\theta}$ é dado por

$$
D_{\theta}=\frac{\boldsymbol{v} \cdot \boldsymbol{\nabla}}{\boldsymbol{\nabla} \cdot \boldsymbol{v}}=\frac{\left(\varepsilon u_{0} R_{0}-q \omega_{0} R\right)}{\left[\left(2 q \omega_{0} R-\varepsilon u_{0} R_{0}\right) \sin \theta+R \partial_{\theta} u_{0}\right]} \frac{R}{R_{0}} \frac{1}{\varepsilon} \frac{\partial}{\partial \theta}
$$

Primeiramente consideremos o caso de rotação puramente toroidal (ETRF). Este tipo de rotação é decorrente da transferência de momento de partículas muito energéticas injetadas no plasma. Tal injeção de partículas é feita em muitos tokamaks com o intuito de aumentar a temperatura do plasma [29]. Devido à alta energia destas, parte do momento é transferida ao plasma causando a rotação deste. A velocidade de equilíbrio [29, 30], neste caso, é dada por

$$
\boldsymbol{v}=v_{T} \hat{e}_{\phi}=\omega_{T}(\Psi) R \hat{e}_{\phi} \approx \omega_{T}(r) R \hat{e}_{\phi}
$$

Se utilizarmos a transformação do sistema de coordenada pseudo-toroidal para o sistema em que estamos trabalhando (equação D.4 apresentado no apêndice D), $\hat{e}_{\phi}=$ $\hat{\boldsymbol{e}}_{\|}+(\varepsilon / q) \hat{\boldsymbol{e}}_{\perp}$, podemos escrever as componentes binormal e paralela da velocidade em 4.51 como

$$
\omega_{0}(r)=\frac{\varepsilon}{q} \frac{R_{0}}{R} u_{0} \quad \text { e } \quad u_{0}(r, \theta)=\omega_{T}(r) \frac{R}{R_{0}} .
$$

Nota-se que a substituição de 4.52 em 4.50 deixa o operador $D_{\theta}$ indefinido. Sendo assim, não é possível, somente com estas informações, obter $p$ e $\rho$. Entretanto consideramos conhecidas tais grandezas. De acordo com [29], tais grandezas foram obtidas a partir da resolução da equação cinética das ondas de deriva [30] e considerando o plasma como um gás perfeito. Sendo assim, a densidade e a pressão de equilíbrio são dadas por

$$
\rho=n(\Psi) m_{i} \exp \left[-\frac{m_{i} v_{T}^{2}}{2 T(\Psi)}\right] \quad \text { e } \quad p=2 \frac{\rho}{m_{i}} T(\Psi)
$$


Este fluxo toroidal de equilíbrio, $\boldsymbol{v}=v_{T} \hat{e}_{\phi}$, cria um campo elétrico de equilíbrio, o qual, de acordo com a equação 4.45, é dado por

$$
\boldsymbol{E}=-\frac{d \Phi}{d \Psi} \hat{\boldsymbol{e}}_{\Psi}=\frac{\varepsilon}{q} B_{\phi} \omega_{T} R \hat{\boldsymbol{e}}_{\Psi}
$$

Há também um outro tipo de rotação, a rotação binormal (rotação toroidal e poloidal), que surge no plasma em decorrência da existência de um campo elétrico. Tal campo elétrico é criado pela difusão ambipolar de partículas carregadas [17]. Neste caso, a velocidade de equilíbrio é uma consequência do movimento de deriva,

$$
\boldsymbol{v}=\boldsymbol{v}_{d}=\frac{\boldsymbol{E} \times \boldsymbol{B}}{B^{2}}=v_{\theta} \hat{e}_{\theta}+v_{\phi} \hat{e}_{\phi}
$$

onde

$$
v_{\theta}=-\frac{E}{B_{\phi}} \quad \text { e } \quad v_{\phi}=-\frac{\varepsilon}{q} v_{\theta} \quad \Longrightarrow \frac{v_{\theta}}{v_{\phi}} \sim \frac{1}{\varepsilon} \gg 1
$$

Pelas equações 4.55 e 4.56, nota-se que o sentido de rotação ocorre principalmente na direção poloidal, pois $v_{\theta} \gg v_{\phi}$. Tal velocidade de equilíbrio também pode ser descrita como

$$
\boldsymbol{v}=\omega_{0} R \hat{\boldsymbol{e}}_{\perp}, \quad \omega_{0}(r)=\omega_{\perp}=-\frac{1}{B R} \frac{d \Phi_{\mathrm{amb}}}{d r}
$$

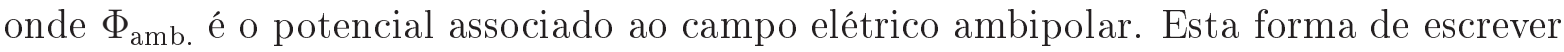
$\boldsymbol{v}$ é a que usaremos nos próximos capítulos.

A densidade e a pressão são dadas pela resolução das equações 4.48 e 4.49, em que o operador $D_{\theta}$ é dado por

$$
D_{\theta}=-\frac{1}{2 \varepsilon} \frac{R}{R_{0}} \frac{\partial}{\partial \theta}
$$

para este tipo de equilíbrio (EBRF).

As soluções de tais equações são dadas por 


$$
\rho=\rho_{r}(r) \frac{R_{0}^{2}}{R^{2}} \quad \text { e } \quad p=p_{r}(r) \frac{R_{0}^{2 \gamma}}{R^{2 \gamma}}
$$

onde utilizamos $\rho_{r}$ e $p_{r}$ para desiginar a parte da densidade e da pressão que depende unicamente da coordenada radial, $r$.

A dependência de $\rho$ e $p$ com $1 / R^{2}$ é explicável fisicamente pelo fato de a rotação do plasma na direção poloidal fazer com que a densidade do plasma tenda a aumentar na borda interior da coluna de plasma. Tal aumento é uma consequência da curvatura geodésica do tokamak, pois a borda interna de um toroide possui um comprimento ao longo da direção toroidal menor do que a borda externa. Tal diferença de comprimento em toroides é conhecida como efeito da toroidicidade. Este pequeno aumento na densidade e na pressão é uma consequência direta da diminuição da velocidade na borda interior e da conservação do fluxo de partículas, $\rho v_{\theta}$ é uma constante com relação a $\theta$.

\subsection{Discussão sobre EBRF}

Se considerarmos a componente binormal ou a componente paralela da equação do balanço de momento, 4.40, para o caso específico de rotação binormal, verifica-se que $\rho$ e $p$ dados por 4.59 não satisfazem tal equação. Isto se deve ao fato de não considerarmos o campo elétrico na equação do balanço de momento. Entretanto, conforme a análise que fazemos a seguir, podemos desprezar o termo $\rho(\boldsymbol{v} \cdot \boldsymbol{\nabla}) \boldsymbol{v}$ na equação 4.40 .

A partir de parâmetros conhecidos para o tokamk TEXTOR [31], estimamos a ordem de grandeza do termo $\rho(\boldsymbol{v} \cdot \boldsymbol{\nabla}) \boldsymbol{v}$ em 4.40 e comparamos com o termos $(\boldsymbol{B} \cdot \boldsymbol{\nabla}) \boldsymbol{B}$. Para tal comparação, utilizamos

$$
v_{\theta} \approx \frac{E_{r}}{B_{\phi}}, \quad B_{\theta} \approx \frac{\mu_{o} I_{P}}{2 \pi a} \quad \text { e } \quad \rho \approx n m_{i}
$$

de forma que

$$
\frac{B_{\theta}^{2}}{\mu_{0}}=\frac{\mu_{0} I_{P}^{2}}{4 \pi^{2} a^{2}} \quad \text { e } \quad \rho v_{\theta}^{2}=\frac{n m_{i} E_{r}^{2}}{B_{\phi}^{2}} .
$$

Tal comparação resulta que 


$$
\frac{B_{\theta}^{2}}{\mu_{0}} \gg \rho v_{\theta}^{2}
$$

para o caso do TEXTOR, o que indica que, pelo menos neste caso, podemos desprezar o termo $\rho v_{\theta}^{2}$ na equação 4.40 .

Torna-se conveniente, então, recorrer às equações da continuidade e da conservação de entropia, 4.41 e 4.42, ou 4.48 e 4.49, cujas soluções são dadas por 4.59. Entretanto, no limite em que $\omega_{0} \rightarrow 0$, devemos ter $\rho=\rho_{r}(r)$ e $p=p_{r}(r)$. Sendo assim, trata-se de um problema caracterizado por uma variação descontínua da densidade e da pressão, sendo este fato, portanto, uma crítica ao modelo proposto. Este modelo certamente não é adequado para descrever o equilíbrio no limite em que o campo elétrico ambipolar é muito pequeno, $O\left(\varepsilon^{2 \gamma}\right)$, com $\gamma>1 / 2$, pois haveria uma descontinuidade abrúpta da ordem de $\varepsilon$ na densidade e na pressão durante a transição entre um campo elétrico ambipolar nulo para um campo ambipolar finito.

Nos capítulos que se seguem utilizaremos principalmente as derivadas com relação à $\theta$ da pressão e da densidade,

$$
\frac{\partial \rho}{\partial \theta}=2 \alpha \frac{R_{0}}{R} \rho \varepsilon \sin \theta \quad \text { e } \quad \frac{\partial p}{\partial \theta}=2 \beta \frac{R_{0}}{R} \gamma p \varepsilon \sin \theta,
$$

onde $\alpha=\beta=1$ se houver rotação de equilíbrio, e $\alpha=\beta=0$ se não houver rotação. Introduzimos as constantes $\alpha$ e $\beta$ para voltarmos ao resultado sem rotação quando tomarmos o limite $\omega_{0} \rightarrow 0$.

\subsection{Sumário sobre equilíbrio}

Esta seção tem o objetivo de organizar de forma clara os resultados encontrados e que serão utilizados no capítulo 6. Conforme ficará claro mais adiante, é conveniente expressar a velocidade de rotação em termos do número de Mach, o qual é definido como a razão entre a velocidade de equilíbrio e a velocidade do som no plasma. Em outras palavras, definimos o número de Mach toroidal como

$$
M_{T}=\frac{u_{0} R_{0}}{c_{s_{0}}}=\frac{\omega_{T}}{\omega_{s 0}}
$$

e o número de Mach binormal como 


$$
M_{\perp}=\frac{\omega_{\perp} R}{c_{s_{0}}}=\frac{\omega_{\perp}}{\omega_{s 0}},
$$

onde $\omega_{s 0}$ é a frequência associada à velocidade do som no plasma, a qual é dada por

$$
c_{s_{0}}=\omega_{s 0} R_{0} \approx \sqrt{\frac{2 \gamma T(\Psi)}{m_{i}}}=\sqrt{\frac{\gamma p}{\rho}},
$$

onde $m_{i}$ é a massa dos íons e $T(\Psi)$ é a temperatura dos íons que consideramos estar em equilíbrio térmico com os elétrons ${ }^{6}$.

A seguir, reunimos as informações mais importantes sobre cada tipo de equilíbrio, as quais serão extensivamente utilizadas nos próximos capítulos. Tais informações colocadas na forma de equações são apresentadas a seguir:

- ENR - Equilíbrio sem rotação

$$
\begin{array}{r}
\omega_{0}=0 \quad \text { e } \quad u_{0}=0 \\
\rho_{0}=\rho_{0}(r) \quad \text { e } \quad p_{0}=p_{0}(r) \\
\frac{\partial \rho_{0}}{\partial \theta}=\frac{\partial p_{0}}{\partial \theta}=0 \\
\bar{\rho}_{0}=\rho_{0}(r)
\end{array}
$$

\section{- ETRF - Equilíbrio com rotação toroidal}

\footnotetext{
${ }^{6}$ Consideramos, neste modelo, que os íons e os elétrons possuem a mesma temperatura.
} 


$$
\begin{array}{r}
\omega_{0}=\frac{\varepsilon}{q} \frac{R_{0}}{R} u_{0} \quad \text { e } \quad u_{0}=\frac{R}{R_{0}} M_{T} \omega_{s 0} \\
\rho_{0}=\rho_{0_{r}}(r) \exp \left(-M_{T}^{2} \frac{R^{2}}{R_{0}^{2}}\right) \quad \text { e } p_{0}=\frac{\omega_{s 0}^{2}}{\gamma} \rho_{0} \\
\frac{\partial \rho_{0}}{\partial \theta}=2 \gamma M_{T}^{2} \frac{R}{R_{0}} \rho_{0} \varepsilon \sin \theta \quad \text { e } \frac{\partial p_{0}}{\partial \theta}=2 M_{T}^{2} \rho_{0} \omega_{s 0}^{2} R_{0} R \\
\bar{\rho}_{0}=\rho_{0_{r}}(r) \mathrm{e}^{-M_{T}^{2}}\left(1+O\left(\varepsilon^{2}\right)\right) \approx \rho_{0_{r}}(r) \mathrm{e}^{-M_{T}^{2}}
\end{array}
$$

- EBRF - Equilíbrio com rotação binormal

$$
\begin{array}{r}
\omega_{0}=M_{\perp} \omega_{s 0} \quad \text { e } \quad u_{0}=0 \\
\rho_{0}=\rho_{0_{r}}(r) \frac{R_{0}^{2}}{R^{2}} \quad \text { e } \quad p_{0}=p_{0 r}(r) \frac{R_{0}^{2 \gamma}}{R^{2 \gamma}} \\
\frac{\partial \rho_{0}}{\partial \theta}=2 \alpha \frac{R_{0}}{R} \rho_{0} \varepsilon \sin \theta \quad \text { e } \frac{\partial p_{0}}{\partial \theta}=2 \beta \gamma \frac{R_{0}}{R} p_{0} \varepsilon \sin \theta, \quad \alpha=\beta=1 \\
\bar{\rho}_{0}=\rho_{0_{r}}(r)\left(1+O\left(\varepsilon^{2}\right)\right) \approx \rho_{0_{r}}(r)
\end{array}
$$

Nas equações 4.67-4.69, definimos a média sobre uma superfície magnética da densidade como

$$
\bar{\rho}_{0}=\frac{\int_{S} d S \rho_{0}(r, \theta)}{\int d S}, \quad d S=r R d \theta d \phi
$$

e voltamos a utilizar o índice 0 para descrever as grandezas de equilíbrio, já que, para os próximos capítulos, a ausência de tal índice poderá causar confusão de notação. 


\section{Capítulo 5}

\section{Física básica dos ZF e GAM}

Neste capítulo, primeiramente derivamos a frequência de ondas acústicas em geometria retangular (uma dimensão) utilizando o sistema de coordenadas cartesianas e considerando o campo magnético e o campo elétrico de equilíbrio nulos e, posteriormente, fazemos a mesma derivação mas em sistemas toroidais com campo magnético não nulo. Descrevemos detalhadamente, do ponto de vista físico, os fluxos zonais (ZF) e os modos acústicos geodésicos (GAM) considerando o equilíbrio sem rotação (ENR).

\subsection{Ondas acústicas em geometria retangular}

A utilização de geometria retangular é a forma mais simples de derivar a relação de dispersão de ondas de som. Apesar de o limite de validade ser restringido à aproximação muito local, tal derivação em geometria retangular é útil para entender, do ponto de vista físico, a origem das ondas de som no plasma.

Ao trabalharmos em geometria retangular, utilizamos coordenadas cartesianas para representar as coordenadas pseudo-toroidais, de forma que $x \rightarrow r, y \rightarrow \theta$ e $z \rightarrow \phi$, conforme mostra a figura 5.1.

A derivação da relação de dispersão para ondas acústicas, em geometria cartesiana, é feita de forma análoga à do capítulo 3. Consideram-se as equações da MHD perturbadas até primeira ordem, porém, como o campo elétrico perturbado é o responsável pelas oscilações, é necessário considerar o plasma como constituído por dois fluidos: o de íons e o elétrons. Representamos as grandezas físicas por $\xi(x, t)=\xi_{0}(x)+\xi_{1}(x, t)$, como fizemos no capítulo 3 e consideramos o campo elétrico de equilíbrio, o campo magnético e a velocidade de equilíbrio nulos, mas o campo elétrico perturbado não nulo: 


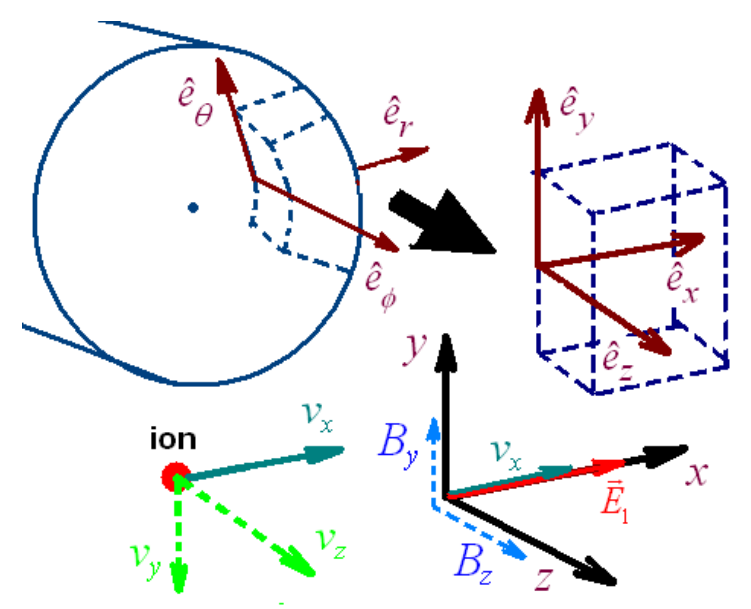

Figura 5.1: Aproximação local das coordenadas pseudo-toroidais para coordenadas cartesianas

$$
\boldsymbol{E}_{\mathbf{0}}=\boldsymbol{B}=\boldsymbol{v}_{\mathbf{0}}=0, \quad \boldsymbol{E}_{1}=-\nabla \Phi_{1}, \quad \boldsymbol{v}_{1}=v_{1} \hat{e}_{x}
$$

Sendo assim, as grandezas físicas perturbadas são da forma $\xi_{1}=C e^{i(k x-\omega t)}$, em que $C$ é uma constante. Tal tipo de função nos permite a seguinte substituição:

$$
\boldsymbol{\nabla} \rightarrow i \boldsymbol{k}=i k \hat{e}_{x} \quad \text { e } \quad \frac{\partial}{\partial t} \rightarrow-i \omega
$$

onde $\boldsymbol{k}$ representa o vetor de propagação de onda, que consideramos na direção $x$ e $\omega$ é a frequência de oscilação. Sendo assim, as equações para os íons a serem resolvidas são dadas por

$$
\begin{gathered}
-i \omega m_{i} n_{0} v_{1}+i k \Phi_{1} e n_{0}+i k p_{i 1}=0, \\
-i \omega n_{i 1}+i k n_{0} v_{i 1}=0,
\end{gathered}
$$

$$
p_{1}=\gamma n_{i 1} T_{i} \quad \mathrm{e}
$$




$$
k^{2} \Phi_{1}=\frac{e}{\varepsilon_{0}}\left(n_{i 1}-\frac{e \Phi_{1} n_{0}}{T_{e}}\right)
$$

onde as grandezas com índice $i$ referem-se aos íons, e $n_{0}=n_{e 0}=n_{i 0}$ é a densidade de íons, ou de elétrons no equilíbrio. Também utilizamos a aproximação de Boltzmann, em que a densidade de elétrons perturbada é $n_{e 1}=n_{0} \exp \left(e n_{0} \Phi_{1} / T_{e}\right)$.

Trata-se de um sistema de quatro equações com quatro incógnitas, $n_{i 1}, p_{i 1}, \Phi_{1}, v_{i 1}$. Determina-se a a frequência de oscilação dessas grandezas, $\omega$, admitindo-se existe uma solução não trivial para elas. Sendo assim, a frequência de oscilação é dada por

$$
\omega^{2}=k^{2}\left(\frac{1}{1+k^{2} \lambda_{D}} \frac{T_{e}}{M}+\gamma \frac{T}{M}\right)
$$

onde $\lambda_{D}=\sqrt{\varepsilon_{0} T_{e}} n_{0} e^{2}$ é o comprimento de Debye, que, em se tratando de ondas de som, pode ser desprezada em primeira aproximação na expressão 5.7 [17]. A velocidade do som no plasma é dada por

$$
\frac{\omega}{k}=c_{s}=\sqrt{\frac{\gamma T+T_{e}}{m_{i}}}
$$

de acordo com a derivação utilizada por F. Chen [17].

Observamos assim que o campo elétrico oscila devido à inércia dos íons e ao movimento destes na direção $x$, ou radial para o caso do tokamak. A velocidade do som também pode ser expressa por

$$
c_{s}=\sqrt{\gamma p / \rho}
$$

onde desprezamos a razão entre a massa do elétron e a do íon, $m / M \sim 0$, de forma que $p \approx n\left(\gamma T+T_{e}\right)$, onde $n$ é a densidade de íons e elétrons considerando a aproximação de quasi-neutralidade e considerando $\gamma_{e}=1$ e $\rho \approx n M$.

Esta relação de dispersão é local, no sentido em que ela não leva em conta o efeito da toroidicidade do campo magnético. Por isso, quando se considera a geometria do conjunto todo, tal como em tokamaks, torna-se inconveniente o uso de coordenadas cartesianas para resolver o problema. No caso de sistemas toroidais, a curvatura geodésica do campo magnético altera o valor da frequência de oscilação do campo elétrico, conforme 
é mostrado a seguir.

\subsection{GAM em sistemas toroidais}

Considerando plasmas sem rotação de equilíbrio, isto é, $\boldsymbol{v}_{\mathbf{0}}=0$, as equações 3.24-3.28, que descrevem a dinâmica do plasma na condição perturbada, são dadas por

$$
\begin{gathered}
-i \omega \rho_{0} \boldsymbol{v}_{\mathbf{1}}+\nabla p_{1}-\boldsymbol{J}_{\mathbf{1}} \times \boldsymbol{B}=0, \\
-i \omega \rho_{1}+\rho_{0} \boldsymbol{\nabla} \cdot \boldsymbol{v}_{\mathbf{1}}=0, \\
\boldsymbol{E}_{\mathbf{1}}+\boldsymbol{v}_{\mathbf{1}} \times \boldsymbol{B}=0 \\
-i \omega p_{1}+\gamma p_{0} \boldsymbol{\nabla} \cdot \boldsymbol{v}_{\mathbf{1}}=0 \quad \mathrm{e} \\
\boldsymbol{\nabla} \cdot \boldsymbol{J}_{\mathbf{1}}=0
\end{gathered}
$$

onde o campo magnético, especificado no capítulo 4 (4.36 e 4.43), é dado por

$$
\left.\boldsymbol{B}=B \hat{\boldsymbol{e}}_{\|}=B_{\phi}\left[(\varepsilon / q) \hat{e}_{\theta}+\hat{e}_{\phi}\right)\right]
$$

e o campo elétrico, considerado como sendo uma função apenas de $\Psi$, é dado por

$$
\boldsymbol{E}_{\mathbf{1}}=E_{1}(\Psi) e^{-i \omega t} \hat{\boldsymbol{e}}_{\Psi}
$$

Como pode ser notado nas equações 5.15 e 5.16, é conveniente utilizar o sistema de coordenadas definido pelos versores $\left(\hat{\boldsymbol{e}}_{\Psi}, \hat{\boldsymbol{e}}_{\|}, \hat{\boldsymbol{e}}_{\perp}\right)$ [32], conforme ficará claro mais adiante. 
Para a derivação da relação de dispersão e das grandezas físicas, é conveniente utilizar o sistema de coordenadas definido pelos versores $\hat{\boldsymbol{e}}_{\Psi}, \hat{\boldsymbol{e}}_{\|}$e $\hat{\boldsymbol{e}}_{\perp}$, conforme indica 5.15 e 5.16. Também é conveniente definir a grandeza $\omega_{1}$, com dimensão de frequência, por

$$
\omega_{1}=\omega_{1}(\Psi)=\frac{E_{1}(\Psi)}{B R} .
$$

Ao multiplicar 5.12 vetorialmente por $\hat{\boldsymbol{e}}_{\|}$, obtemos a componente binormal de $\boldsymbol{v}_{1}$, porém, ao multiplicar 5.10 escalarmente por $\hat{\boldsymbol{e}}_{\|}$, obtemos a componente paralela de $\boldsymbol{v}_{\mathbf{1}}$, de forma que

$$
\boldsymbol{v}_{\mathbf{1}}=\omega_{1} R \hat{\boldsymbol{e}}_{\perp}+u_{1} R_{0} \hat{\boldsymbol{e}}_{\|}
$$

onde

$$
\omega_{1}=-\frac{1}{B R} \frac{d \Phi_{1}}{d r} \quad \text { e } \quad u_{1}=-i \frac{1}{R_{0}} \frac{\nabla_{\|} p_{1}}{\rho_{0} \omega}
$$

em que também utilizamos as identidades vetoriais A.1 e A.2 para derivar 5.18.

De 5.11 obtemos

$$
\rho_{1}=-i \frac{\rho_{0}}{\omega} \nabla \cdot \boldsymbol{v}_{1}
$$

que, ao ser utilizada em conjunto com a relação

$$
\frac{\gamma p_{0}}{\rho_{0}}=c_{s_{0}}^{2}=\omega_{s 0}^{2} R_{0}^{2}, \quad \text { onde } \quad \omega_{s 0}=\omega_{s 0}(\Psi)
$$

em 5.13, nos fornece

$$
p_{1}=\omega_{s 0}^{2} R_{0}^{2} \rho_{1} .
$$

Ao tomar as componentes $\hat{\boldsymbol{e}}_{\Psi}$ e $\hat{\boldsymbol{e}}_{\perp}$ de 5.10 , obtemos as principais componentes de $\boldsymbol{J}_{1}$ :

$$
J_{1 \Psi}=\frac{1}{B}\left(-i \rho_{0} \omega_{1} \omega R+\nabla_{\perp} p_{1}\right) \quad \text { e } \quad J_{1 \perp}=-\frac{1}{B} \nabla_{\Psi} p_{1} .
$$


Utilizando o teorema da divergência de Gauss, A.14, e a condição de quasineutralidade do plasma, 5.14, obtemos a equação que fornece a relação de dispersão, mais explicitamente:

$$
\int_{V} \boldsymbol{\nabla} \cdot \boldsymbol{J}_{\mathbf{1}} d V=\int_{S} \boldsymbol{J}_{\mathbf{1}} \cdot d \boldsymbol{S}=0
$$

Convenientemente, escolhe-se a superfície $S$ como sendo uma superfície magnética, $S=$ $r d \theta R d \phi \Longrightarrow d \boldsymbol{S}=\operatorname{Rr} d \theta d \phi \hat{\boldsymbol{e}}_{\Psi}$, de forma que

$$
\int_{S} \boldsymbol{J}_{\mathbf{1}} \cdot d \boldsymbol{S}=\int_{0}^{2 \pi} r d \phi \int_{0}^{2 \pi} d \theta R J_{1 \Psi}=0
$$

A equação 5.24, além de fornecer a relação de dispersão, conforme será verificado mais adiante, também nos diz que a média sobre uma superfície magnética, $\Psi=$ const., da densidade de corrente é nula. Tal média é dada por

$$
\left\langle J_{1 \Psi}\right\rangle=\frac{\int_{S} \boldsymbol{J}_{\mathbf{1}} \cdot d \boldsymbol{S}}{\int_{S} d S}=\frac{1}{2 \pi R_{0}} \int_{0}^{2 \pi} d \theta R J_{1 \Psi}=0 .
$$

As equações 5.18-5.22 e 5.25 constituem um sistema completo que, ao ser resolvido, fornece a frequência de oscilação e as autofunções dos ZF e GAMs. Ao substituir 5.19 em 5.21, utilizando 5.18, obtém-se uma equação diferencial para $p_{1}$;

$p_{1}=-i \frac{\rho_{0} \omega_{s 0}^{2} R_{0}^{2}}{\omega}\left(2 \omega_{1} \sin \theta+\frac{\varepsilon}{q} \frac{R_{0}}{R} \sin \theta+\frac{1}{q} \frac{\partial u_{1}}{\partial \theta}\right) \approx-i \frac{\rho_{0} \omega_{s 0}^{2} R_{0}^{2}}{\omega}\left(2 \omega_{1} \sin \theta+\frac{1}{q} \frac{\partial u_{1}}{\partial \theta}\right)$,

onde utilizamos que

$$
u_{1}=-i \frac{\nabla_{\|} p_{1}}{\rho_{0} \omega R_{0}} \quad \text { e } \quad \boldsymbol{v}_{\mathbf{1}}=\omega_{1} R \hat{\boldsymbol{e}}_{\perp}+u_{1} R_{0} \hat{\boldsymbol{e}}_{\|}
$$

e a relação D.8. Ao utilizar D.4 bem como a simetria no ângulo azimutal, $\phi$, podemos reescrever 5.26 como 
$p_{1}=-i \frac{\rho_{0} \omega_{s 0}^{2} R_{0}^{2}}{\omega}\left(2 \omega_{1} \sin \theta-\frac{i}{q^{2} \rho_{0} R_{0}^{2} \omega} \frac{\partial^{2} p_{1}}{\partial \theta^{2}}\right) \Longrightarrow\left(1+\frac{\omega_{s 0}^{2}}{q^{2} \omega^{2}} \frac{\partial^{2}}{\partial \theta^{2}}\right) p_{1}=-\frac{2 i \omega_{s 0}^{2} R_{0}^{2}}{\omega} \omega_{1} \sin \theta$,

e cuja solução para $p_{1}$ é dada por

$$
p_{1}=\frac{2 i q^{2} \Omega}{1-q^{2} \Omega^{2}} \rho_{0} \omega_{s 0} \omega_{1} R_{0}^{2} \sin \theta,
$$

onde utilizamos a frequência adimensional definida por

$$
\Omega=\frac{\omega}{\omega_{s 0}}
$$

para simplificar a notação.

Ao substituir 5.29 em 5.22, obtemos a componente $\hat{\boldsymbol{e}}_{\Psi}$ da densidade de corrente,

$$
J_{1 \Psi} \approx \frac{-i \rho_{0} \omega_{1} \omega_{s 0} \Omega R}{B_{\phi 0} R_{0}}\left(R+\frac{1}{\varepsilon} \frac{2 q^{2} R_{0}}{1-q^{2} \Omega^{2}} \cos \theta\right)
$$

onde utilizamos também D.4.

Ao substituir 5.31 em 5.25, obtemos a relação de dispersão para a frequência de oscilação:

$$
\int_{0}^{2 \pi} d \theta R\left(\frac{-i \rho_{0} \omega_{1} \omega_{s 0} \Omega R}{B_{\phi 0} R_{0}}\right)\left(R+\frac{1}{\varepsilon} \frac{2 q^{2} R_{0}}{1-q^{2} \Omega^{2}} \cos \theta\right) \Longrightarrow \Omega\left(1+\frac{2 q^{2}}{1-q^{2} \Omega^{2}}\right)=0
$$

em que foi utilizada a relação D.16 para resolver a integral. Tal relação de dispersão possui as seguintes soluções

$$
\Omega_{z, \mathrm{nr}}=0 \quad \text { e } \quad \Omega_{g, \mathrm{nr}}=f_{g, \mathrm{nr}}, \quad \text { onde } \quad f_{g, \mathrm{nr}}=\sqrt{2+\frac{1}{q^{2}}}
$$

que correspondem, respectivamente, a frequência dos ZF e a frequência dos GAM.

A seguir explicamos o mecanismo físico dos GAM. As grandezas físicas para tal explicação são obtidas tomando-se a parte real das equações anteriores, uma vez que somente 
a parte real tem um significado físico, de forma que explicitamos a dependência temporal de tais grandezas físicas. Utilizamos notação " $\tilde{\xi}$ ", para expressar a parte de $\xi$ dependente somente das coordenadas.

Um campo elétrico, na direção perpendicular as superfícies magnéticas,

$$
E_{1}=\tilde{E}_{1}(\Psi) \cos (\omega t)
$$

(parte real de 5.16) juntamente com o campo magnético, $\boldsymbol{B}=B \hat{\boldsymbol{e}}_{\|}$, produz um movimento de deriva no plasma,

$$
\boldsymbol{v}_{\mathbf{1}}=\frac{\tilde{E}_{1}}{B}\left[\hat{\boldsymbol{e}}_{\perp}-\frac{1}{q} \cos \theta \hat{\boldsymbol{e}}_{\|}\right] \cos (\omega t)
$$

(parte real de 5.18, utilizando também 5.29), na direção binormal. Entretanto a toroidicidade do tokamak produz um fluxo na direção do campo elétrico $\left(-\frac{1}{q} \cos \theta\right)$, que faz com que haja uma perturbação na densidade do plasma,

$$
\rho_{1}=-\sqrt{2+\frac{1}{q^{2}}} \frac{\tilde{E}_{1}}{B R \omega_{s 0}} \rho_{0}(\Psi) \sin \theta \sin (\omega t)
$$

proporcional ao divergente da velocidade, conforme 5.19. Como a pressão está diretamente relacionada com a densidade, também ocorre uma perturbação da pressão,

$$
p_{1}=-\sqrt{2+\frac{1}{q^{2}}} \frac{\tilde{E}_{1}}{B R} \rho_{0} \omega_{s 0} R_{0}^{2} \sin \theta \sin (\omega t)
$$

(as equações 5.36 e 5.37 foram obtidas de 5.21, 5.29 e 5.33). Tanto a velocidade de deriva, quanto a pressão produzem uma corrente superficial que transporta carga elétrica através das superfícies magnéticas de forma a anular o campo elétrico inicial, $\boldsymbol{E}_{\mathbf{1}}$. Isto pode ser visto na equação de momento, 5.10, cuja componente binormal de $\boldsymbol{J}_{1}$ pode ser escrita como

$$
J_{1 \Psi}=\frac{1}{B}\left(\rho_{0} \frac{\partial v_{1_{\perp}}}{\partial t}+\nabla_{\perp} p_{1}\right)
$$

que, de acordo com 5.31, pode ser mais explicitamente dada por 


$$
J_{1 \Psi}=-\rho_{0} \omega_{s 0} R_{0} \frac{\tilde{E}_{1}}{B^{2}}\left(1-\frac{1}{\varepsilon} \cos \theta\right) \sin (\omega t) .
$$

Devido à inércia do plasma, surge este modo de oscilação, cuja frequência possui uma parte relacionada a ondas de som no plasma e outra parte relacionada à curvatura geodésica do campo magnético. Em outras palavras, os GAM estão relacionados diretamente com a divergência da velocidade perturbada,

$$
\boldsymbol{\nabla} \cdot \boldsymbol{v}_{\mathbf{1}}=\frac{\tilde{E}_{1}}{B R}\left(2+\frac{1}{q^{2}}\right) \sin \theta
$$

que, de acordo com 5.35 e D.21, não se anula. A incompressibilidade do plasma produz esses modos que estão diretamente relacionados com a frequência de ondas sonoras, $\omega_{s 0}$, e com a curvatura geodésica do campo magnético,ou seja,

$$
\omega_{g, \mathrm{nr}}=f_{g, \mathrm{nr}} \omega_{s 0}, \quad\left(f_{g, \mathrm{nr}}^{2}=2+\frac{1}{q^{2}}\right)
$$

de forma que o termo $\frac{1}{q^{2}}$ está relacionado com as ondas de som e o termo 2, com a curvatura geodésica do campo magnético. Isto pode ser visto pela fórmula derivada em [16], que é válida para um sistema toroidal genérico,

$$
\begin{array}{r}
\omega^{2} \int\left|\rho_{1}\right|^{2} \jmath d S=\frac{\gamma p_{0}}{\rho_{0}}\left[\frac{\left|\int \rho_{1} B^{-4}(\nabla \Psi \times \boldsymbol{B}) \cdot \nabla B^{2} \jmath d S\right|^{2}}{\int\|\nabla \Psi\|^{2} B^{-2} \jmath d S}+\int B^{-2}\left|\boldsymbol{B} \cdot \nabla \rho_{1}\right|^{2} \jmath d S\right] \\
\Omega^{2}=\frac{R_{0}^{2}}{\int\left|\rho_{1}\right|^{2} \jmath d S}\left[\frac{\left|\int \rho_{1} B^{-3}\|\nabla \Psi\| \nabla_{\perp} B^{2} \jmath d S\right|^{2}}{\int\|\nabla \Psi\|^{2} B^{-2} \jmath d S}+\int B^{-2}\left|B \nabla_{\|} \rho_{1}\right|^{2} \jmath d S\right],
\end{array}
$$

onde $\jmath$ é o jacobiano referente ao sistema de coordenadas adotado. O primeiro termo do lado direito de 5.42 é devido ao movimento ao longo da direção binormal e está associado à curvatura geodésica do campo magnético $\left(\nabla_{\perp} B^{2}\right)$. Já o segundo termo representa ondas de som comum se propagando ao longo das linhas de campo magnético $\left(\nabla_{\|} \rho_{1}\right)$.

Como $\Psi, \boldsymbol{B}$ e $\jmath$ são determinados pela geometria do sistema em questão, só precisamos 
conhecer a grandeza $\rho_{1}$ para calcular a frequência de oscilação $\Omega$. Para um tokamak de secção circular e alta razão de aspecto, temos

$$
\Psi=h(r), \quad \boldsymbol{B} \approx B_{\phi 0}(r)\left[1-(\varepsilon / 2)\left(e^{i \theta}+e^{-i \theta}\right)\right] \hat{\boldsymbol{e}}_{\|} \quad \mathrm{e} \quad \jmath=r R_{0}\left[1+(\varepsilon / 2)\left(e^{i \theta}+e^{-i \theta}\right)\right]
$$

e $\rho_{1}$ pode ser desenvolvido em série de Fourier,

$$
\rho_{1}=e^{-i \omega t} \sum_{m=-\infty}^{\infty} \rho_{1 m}(r) e^{i m \theta}
$$

na coordenada poloidal $\theta$. Consideramos também que há simetria axial, ou seja, $n=0$, do contrário

$$
\rho_{1}=e^{-i \omega t} \sum_{n=-\infty}^{\infty} \sum_{m=-\infty}^{\infty} \rho_{1 m n}(r) e^{i(m \theta+n \phi)}
$$

Ao substituir 5.43 e 5.44 em 5.42, obtemos

$$
\begin{array}{r}
\Omega^{2} \approx R_{0}^{2}\left(\int_{0}^{2 \pi} d \theta\left|\sum_{m=-\infty}^{\infty} \rho_{1 m}(r)^{i m \theta}\right|^{2}\right)^{-1} \times \\
\left\{\frac{1}{R_{0}^{2}}\left|\int_{0}^{2 \pi} d \theta i\left(\sum_{m=-\infty}^{\infty} \rho_{1 m} e^{i(m+1) \theta}-\sum_{m=-\infty}^{\infty} \rho_{1 m} e^{i(m-1) \theta}\right)\right|^{2}+\right. \\
\left.\frac{1}{q^{2} R_{0}^{2}} \int_{0}^{2 \pi} d \theta\left|i\left(\sum_{m=-\infty}^{\infty} \rho_{1 m} e^{i(m+1) \theta}-\sum_{m=-\infty}^{\infty} \rho_{1 m} e^{i(m-1) \theta}\right)\right|^{2}\right\} .
\end{array}
$$

Ao supor que $\rho_{1} \propto \sin \theta$, ou seja, as duas únicas componentes de $\rho_{1 m}$ são $\rho_{1-1}$ e $\rho_{11}$, com $\rho_{1-1}=-\rho_{11}$, a equação 5.46 pode ser escrita como

$$
\Omega^{2}=\left(2\left|\rho_{11}\right|^{2}\right)^{-1}\left\{2\left|\rho_{11}\right|^{2}+2\left|\rho_{11}\right|^{2}+2\left|\rho_{11}\right|^{2} \frac{1}{q^{2}}\right\}=2+\frac{1}{q^{2}}
$$

Portanto, conclui-se com base em 5.42, 5.46 e 5.47 que os GAM são causados pelo acoplamento dos modos $n=0, m= \pm 1$ da densidade (ou pressão) com os modos $n=0$, 


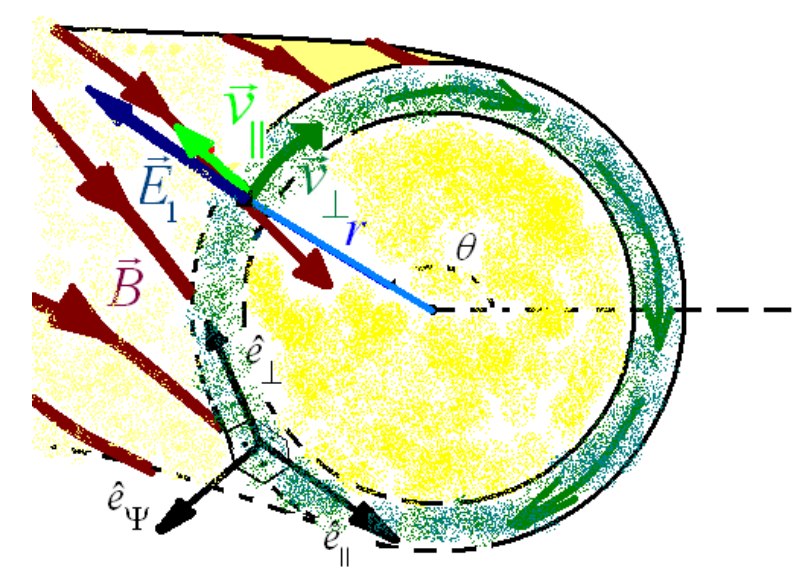

Figura 5.2: Grandezas físicas relevantes para a descrição dos GAM no tokamak

$m=0$ do campo elétrico e $n=0, m= \pm 1$ do campo magnético, onde $n$ é o modo referente ao ângulo toroidal $(\phi)$ e $m$ ao ângulo poloidal $(\theta)$. Além disso, vemos que o termo 2 de $\Omega^{2}$ está relacionado com a curvatura geodésica do campo magnético, enquanto o termo $1 / q^{2}$ são ondas acústicas em plasmas magnetizados. Se não considerarmos alta razão de aspecto e tokamaks com simetria toroidal, claramente vemos que outros modos além de $n=0$ e $m= \pm 1$ de $\rho_{1}\left(p_{1}\right)$ influenciariam nos cálculos. Na figura 5.2, mostramos o esquema dos GAM em um tokamak do ponto de vista geométrico.

Por outro lado, os ZF surgem quando o plasma se comporta como um fluido compressível, ou seja, se

$$
\boldsymbol{\nabla} \cdot \boldsymbol{v}_{1}=0
$$

de acordo com 5.19 e 5.21 teríamos $\rho_{1}=p_{1}=0=$ e, de acordo com 5.22 e 5.32 , teríamos também $J_{1 \Psi}=J_{1 \perp}=0$, sendo assim, não há oscilações temporais do campo elétrico, assim como de outras grandezas físicas. Entretanto a existência de um fluxo binormal e da não divergência deste fluxo é compensada com um fluxo da direção paralela ao campo magnético. Se decompusermos a velocidade perturbada na componente binormal e paralela ao campo magnético, ou seja,

$$
\boldsymbol{v}_{\mathbf{1}}=\omega_{1} R \hat{\boldsymbol{e}}_{\perp}+u_{1} R_{0} \hat{\boldsymbol{e}}_{\|}
$$

de acordo com D.21 teríamos $u_{1}=2 q \omega_{1} \cos \theta$. Este é o fluxo de retorno ao longo das linhas 
de campo magnético causado pela presença do fluxo binormal e pela incompressibilidade do plasma. Desta forma temos

$$
\boldsymbol{v}_{\mathbf{1}}=\frac{\tilde{E}_{1}}{B}\left[\hat{\boldsymbol{e}}_{\perp}+2 q \cos \theta \hat{\boldsymbol{e}}_{\|}\right] \cos (\omega t)
$$

Este resultado está de acordo com [23], que fornece a mesma explicação física, porém de forma não tão clara.

Ao obtermos as grandezas físicas perturbadas $\left(\rho_{1}, p_{1}\right.$ e $\left.\boldsymbol{v}_{1}\right)$ neste capítulo, notamos a dependência $\operatorname{com} \sin \theta$ ou $\cos \theta$ destas. Tal dependência surgiu naturalmente ao encontrarmos os autovalores ( $\omega$ ou $\Omega$ ) e as autofunções associadas a eles $\left(\rho_{1}, p_{1}\right.$ e $\left.\boldsymbol{v}_{\mathbf{1}}\right)$. No próximo capítulo, o mesmo problema com rotação de equilíbrio é resolvido, entretanto, como os cálculos se tornam relativamente mais complexos, adotamos uma metodologia apropriada para lidar com esta complexidade. Esta metodologia consiste em testar soluções tentativas da forma $\xi(r, \theta)=\xi_{s}(r) \sin \theta+\xi_{c}(r) \cos \theta$ para as grandezas perturbadas. 


\section{Capítulo 6}

\section{Efeito da rotação nos ZF e GAM em tokamaks de secção circular}

Conforme descrito no final do capítulo anterior, adotamos a metodologia de supor soluções tentativas da forma $\xi(r, \theta)=\xi_{s}(r) \sin \theta+\xi_{c}(r) \cos \theta$ para $\boldsymbol{v}_{\mathbf{1}}$, $\rho_{\mathbf{1}}$ e $p_{1}$, com o intuito de encontrar as frequências dos ZF e GAM na presença de rotação de equilíbrio. Consideramos, ainda, apenas o modo $n=m=0$ do campo elétrico perturbado. Termos de ordem $O\left(\varepsilon^{2}\right)$ são desprezados em comparação com termos de ordem 1 e $\varepsilon$, de forma que ainda consideramos a aproximação de alta razão de aspecto neste capítulo.

Partimos das equações da MHD ideal derivadas no capítulo 3, 3.24-3.28, que podem ser escritas como

$$
\begin{gathered}
\int_{0}^{2 \pi} d \theta R J_{1 \Psi}=0 \\
-i \omega \boldsymbol{v}_{\mathbf{1}}+\boldsymbol{F}+\frac{1}{\rho_{0}} \nabla p_{1}-\frac{1}{\rho_{0}} \boldsymbol{J}_{\mathbf{1}} \times \boldsymbol{B}=0 \\
-i \omega \rho_{1}+G+\rho_{0} \boldsymbol{\nabla} \cdot \boldsymbol{v}_{\mathbf{1}}=0 \\
-i \omega p_{1}+H+\rho_{0} \omega_{s 0}^{2} R_{0}^{2} \boldsymbol{\nabla} \cdot \boldsymbol{v}_{\mathbf{1}}=0
\end{gathered}
$$


sendo que a velocidade e o campo magnético, conforme mostrado anteriormente (5.15, $5.18,4.36$ e 4.44), são dados por

$$
\begin{aligned}
\boldsymbol{v}_{i}=\omega_{i} R \hat{\boldsymbol{e}}_{\perp}+u_{i} R_{0} \hat{\boldsymbol{e}}_{\|}, \quad i=0,1 \mathrm{e} \\
\boldsymbol{B} \approx B_{\phi 0} \frac{R_{0}}{R} \hat{\boldsymbol{e}}_{\|},
\end{aligned}
$$

em que $\omega_{i}=\omega_{i}(r)$ e $u_{i}=u_{i}(r, \theta)$ para tokamaks de secção circular.

Nas equações 6.2-6.4, os termos $\boldsymbol{F}, G$ e $H$ são dados por

$$
\begin{array}{r}
\boldsymbol{F}=\boldsymbol{F}\left(\boldsymbol{v}_{\mathbf{0}}, \boldsymbol{v}_{\mathbf{1}}, \rho_{0}, \rho_{1}\right)=\left(\boldsymbol{v}_{\mathbf{0}} \cdot \boldsymbol{\nabla}\right) \boldsymbol{v}_{\mathbf{1}}+\left(\boldsymbol{v}_{\mathbf{1}} \cdot \boldsymbol{\nabla}\right) \boldsymbol{v}_{\mathbf{0}}+\frac{\rho_{1}}{\rho_{0}}\left(\boldsymbol{v}_{\mathbf{0}} \cdot \boldsymbol{\nabla}\right) \boldsymbol{v}_{\mathbf{0}} \\
G=G\left(\boldsymbol{v}_{\mathbf{0}}, \boldsymbol{v}_{\mathbf{1}}, \rho_{0}, \rho_{1}\right)=\left(\boldsymbol{v}_{\mathbf{0}} \cdot \boldsymbol{\nabla}\right) \rho_{1}+\left(\boldsymbol{v}_{\mathbf{1}} \cdot \boldsymbol{\nabla}\right) \rho_{0}+\rho_{1} \boldsymbol{\nabla} \cdot \boldsymbol{v}_{\mathbf{0}} \quad \mathrm{e} \\
H=H\left(\boldsymbol{v}_{\mathbf{0}}, \boldsymbol{v}_{\mathbf{1}}, \omega_{s 0}, \rho_{0}, p_{1}\right)=\left(\boldsymbol{v}_{\mathbf{0}} \cdot \boldsymbol{\nabla}\right) p_{1}+\frac{1}{\gamma}\left(\boldsymbol{v}_{\mathbf{1}} \cdot \boldsymbol{\nabla}\right)\left(\omega_{s 0}^{2} \rho_{0}\right)+\gamma p_{1} \boldsymbol{\nabla} \cdot \boldsymbol{v}_{\mathbf{0}} .
\end{array}
$$

A equação 6.1, a qual representa a quasineutralidade de uma superfície magnética, é análoga ao caso sem rotação derivada no capítulo anterior, 5.25.

Analogamente ao capítulo anterior, tomamos a componente binormal de 6.2 para obter $J_{1 \Psi}$,

$$
J_{1 \Psi}=\frac{R}{B_{\phi 0} R_{0}}\left(-i \omega \omega_{1} \rho_{0} R+\rho_{0} F_{\perp}+\nabla_{\perp} p_{1}\right)
$$

que, ao ser substituida em 6.1, fornece

$$
\int_{0}^{2 \pi} d \theta \rho_{0} R^{2}\left(-i \omega \omega_{1} R+F_{\perp}+\frac{\nabla_{\perp} p_{1}}{\rho_{0}}\right)=0,
$$

onde $F_{\perp}=\hat{\boldsymbol{e}}_{\perp} \cdot \boldsymbol{F}$ e $\nabla_{\perp}=-1 /\left(\varepsilon R_{0}\right) \partial_{\theta}$, conforme descrito no apêndice D.

A componente paralela a $\boldsymbol{B}$ de 6.2 fornece a componente paralela da velocidade perturbada,

$$
u_{1}=-i\left(\frac{F_{\|}}{\omega R_{0}}+\frac{\nabla_{\|} p_{1}}{\rho_{0} \omega R_{0}}\right)
$$

analogamente à forma como foi feita no capítulo anterior para obter a equação 5.18. 
Nesta equação, $F_{\|}=\boldsymbol{F} \cdot \hat{\boldsymbol{e}}_{\|}$e $\nabla_{\|}=1 /\left(q R_{0}\right) \partial_{\theta}$ (equação D.30 do apêndice D).

Das equações 6.3 e 6.4, obtêm-se, respectivamente, a densidade perturbada e a pressão perturbada:

$$
\begin{aligned}
& \rho_{1}=-i\left(\frac{\rho_{0}}{\omega} \nabla \cdot \boldsymbol{v}_{1}+\frac{G}{\omega}\right) \mathrm{e} \\
& p_{1}=-i\left[\frac{\rho_{0} \omega_{s 0}^{2} R_{0}^{2}}{\omega} \nabla \cdot \boldsymbol{v}_{\mathbf{1}}+\frac{H}{\omega}\right] .
\end{aligned}
$$

As equações 6.8-6.11 constituem um sistema completo nas incógnitas $\omega, u_{1}, \rho_{1}$ e $p_{1}$. Como os ZF e GAM estão associados ao número poloidal $m=1$ para $u_{1}, \rho_{1}$ e $p_{1}$, propomos, para essas grandezas, soluções da forma,

$$
\xi(r, \theta)=\xi_{s}(r) \sin \theta+\xi_{c}(r) \cos \theta
$$

de forma que tais equações podem ser reescritas como

$$
\begin{gathered}
-i \Omega \omega_{1} \omega_{s 0} R_{0}+F_{\perp 0}+\varepsilon F_{\perp c}-\frac{p_{1 s}}{\bar{\rho}_{0} R_{0}}=0, \\
u_{1 s}=\frac{-i}{\Omega \omega_{s 0} R_{0}}\left(F_{\| s}-\frac{p_{1 c}}{q \bar{\rho}_{0} R_{0}}\right) \\
u_{1 c}=\frac{-i}{\Omega \omega_{s 0} R_{0}}\left(F_{\| c}+\frac{p_{1 s}}{q \bar{\rho}_{0} R_{0}}\right) \\
\rho_{1 s}=\frac{-i}{\Omega \omega_{s 0}}\left[\left(2 \omega_{1}-\frac{u_{1 c}}{q}\right) \bar{\rho}_{0}+G_{s}\right] \\
\rho_{1 c}=\frac{-i}{\Omega \omega_{s 0}}\left(\frac{u_{1 s}}{q} \bar{\rho}_{0}+G_{c}\right)
\end{gathered}
$$




$$
\begin{gathered}
p_{1 s}=\frac{-i}{\Omega \omega_{s 0}}\left[\left(2 \omega_{1}-\frac{u_{1 c}}{q}\right) \bar{\rho}_{0} \omega_{s 0}^{2} R_{0}^{2}+H_{s}\right] \mathrm{e} \\
p_{1 c}=\frac{-i}{\Omega \omega_{s 0}}\left(\frac{u_{1 s}}{q} \bar{\rho}_{0} \omega_{s 0}^{2} R_{0}^{2}+H_{c}\right),
\end{gathered}
$$

onde as grandezas com índices $0, c$ e $s$ representam, respectivamente, as componentes de $(\cos \theta)^{0}, \cos \theta$ e $\sin \theta$. Fizemos uso da propriedade

$$
f(r) \sin \theta+g(r) \cos \theta=0 \quad \forall \quad \theta \quad \Longrightarrow f(r)=0 \quad \text { e } \quad g(r)=0,
$$

para escrever as equações $6.12-6.18$ e utilizamos a frequência adimensional, $\Omega=\omega / \omega_{s 0}$.

Aproximamos a densidade de equilíbrio por

$$
\rho_{0} \approx \bar{\rho}_{0}=<\rho_{0}>=\frac{\int d S \rho_{0}}{\int d S}=\frac{1}{2 \pi R_{0}} \int_{0}^{2 \pi} d \theta R \rho_{0},
$$

cujo erro é dado por

$$
\sigma_{\rho_{0}}=\left|\rho_{0}-\bar{\rho}_{0}\right|
$$

Antes de resolver as equações 6.12-6.18, é conveniente simplificá-las definindo as seguintes grandezas adimensionais:

$$
\begin{gathered}
\overline{\boldsymbol{F}}=\frac{\boldsymbol{F}}{\omega_{1} \omega_{s 0} R_{0}}, \quad \bar{G}=\frac{G}{\bar{\rho}_{0} \omega_{1}}, \quad \bar{H}=\frac{H}{\bar{\rho}_{0} \omega_{1} \omega_{s 0}^{2} R_{0}^{2}} \\
\overline{p_{1}}=\frac{p_{1}}{\bar{\rho}_{0} \omega_{1} \omega_{s 0} R_{0}^{2}}, \quad \overline{u_{1}}=\frac{u_{1}}{\omega_{1}} \quad \text { e } \quad \overline{\rho_{1}}=\frac{\rho_{1} \omega_{s 0}}{\bar{\rho}_{0} \omega_{1}}
\end{gathered}
$$

onde ficam subentendidos os índices $\perp, \|, 0, s$ e $c$, na equação 6.21. Sendo assim, as equações 6.12 a 6.18 são reescritas como

$$
-i \Omega+\bar{F}_{\perp 0}+\varepsilon \bar{F}_{\perp c}-\bar{p}_{1 s}=0,
$$




$$
\begin{gathered}
\bar{u}_{1 s}=\frac{-i}{\Omega}\left(\bar{F}_{\| s}-\frac{\bar{p}_{1 c}}{q}\right), \\
\bar{u}_{1 c}=\frac{-i}{\Omega}\left(\bar{F}_{\| c}+\frac{\bar{p}_{1 s}}{q}\right), \\
\bar{\rho}_{1 s}=\frac{-i}{\Omega}\left[\left(2-\frac{\bar{u}_{1 c}}{q}\right)+\bar{G}_{s}\right], \\
\bar{\rho}_{1 c}=\frac{-i}{\Omega}\left(\frac{\bar{u}_{1 s}}{q}+\bar{G}_{c}\right), \\
\bar{p}_{1 s}=\frac{-i}{\Omega}\left[\left(2-\frac{\bar{u}_{1 c}}{q}\right)+\bar{H}_{s}\right] \mathrm{e} \\
\bar{p}_{1 c}=\frac{-i}{\Omega}\left(\frac{\bar{u}_{1 s}}{q}+\bar{H}_{c}\right) .
\end{gathered}
$$

Nas seções que se seguem, resolvemos as equações $6.22-6.28$ para cada tipo de equilíbrio; ENR, ETRF e EBRF.

\subsection{Equilíbrio sem rotação - ENR}

Para o equilíbrio sem rotação, ENR, temos

$$
\boldsymbol{v}_{\mathbf{0}}=\frac{\partial \rho_{0}}{\partial \theta}=\frac{\partial p_{0}}{\partial \theta}=0 \Longrightarrow \overline{\boldsymbol{F}}=\bar{G}=\bar{H}=0
$$

de acordo com 6.6. Sendo assim as equações 6.22-6.28 ficam

$$
-i \Omega-\bar{p}_{1 s}=0
$$




$$
\begin{gathered}
\bar{u}_{1 s}=\frac{i \bar{p}_{1 c}}{q \Omega}, \\
\bar{u}_{1 c}=\frac{-i \bar{p}_{1 s}}{q \Omega}, \\
\bar{\rho}_{1 s}=\frac{-i}{\Omega}\left(2-\frac{\bar{u}_{1 c}}{q}\right), \\
\bar{\rho}_{1 c}=\frac{-i \bar{u}_{1 s}}{q \Omega}, \\
\bar{p}_{1 s}=\frac{-i}{\Omega}\left(2-\frac{\bar{u}_{1 c}}{q}\right) \\
\mathrm{e} \\
\bar{p}_{1 c}=\frac{-i}{\Omega} \frac{\bar{u}_{1 s}}{q} .
\end{gathered}
$$

Ao substituir 6.36 e 6.35 em 6.31 e 6.32 , respectivamente, obtemos $\bar{u}_{1}$,

$$
\begin{gathered}
\bar{u}_{1}=\bar{u}_{1 s} \sin \theta+\bar{u}_{1 c} \cos \theta \\
\bar{u}_{1 s}=0 \quad \text { e } \quad \bar{u}_{1 c}=\frac{2 q}{1-q^{2} \Omega^{2}}
\end{gathered}
$$

que, ao ser substituído em 6.33-6.36, nos permite obter $\bar{\rho}_{1}$ e $\bar{p}_{1}$,

$$
\begin{array}{r}
\bar{\rho}_{1}=\bar{\rho}_{1 s} \sin \theta+\bar{\rho}_{1 c} \cos \theta \\
\bar{\rho}_{1 s}=\frac{2 i q^{2} \Omega}{1-q^{2} \Omega^{2}}, \quad \bar{\rho}_{1 c}=0 \quad \mathrm{e}
\end{array}
$$




$$
\begin{array}{r}
\bar{p}_{1}=\bar{p}_{1 s} \sin \theta+\bar{p}_{1 c} \cos \theta \\
\bar{p}_{1 s}=\bar{\rho}_{1 s} \quad \text { e } \quad \bar{p}_{1 c}=0 .
\end{array}
$$

A substituição de $\bar{p}_{1 s}$ em 6.30 leva a equação para a frequência $\Omega$,

$$
\Omega\left(1+\frac{2 q^{2}}{1-q^{2} \Omega^{2}}\right)=0
$$

cujas soluções são: $\Omega=0$, correspondentes à frequência dos ZF e $\Omega=2+1 / q^{2}$, correspondente à frequência dos GAM. Estes resultados são confirmados pela equação 5.33, $\Omega_{z, \mathrm{nr}}=0$ e $\Omega_{g, \mathrm{nr}}=f_{g, \mathrm{nr}}=\sqrt{2+1 / q^{2}}$, do capítulo anterior.

O erro da aproximação $\rho_{0} \rightarrow \bar{\rho}_{0}$ é $\sigma_{\rho_{0}}=0$, de acordo com 6.19, 6.20 e D.20.

\subsection{Equilíbrio com rotação toroidal - ETRF}

Para o equilíbrio com rotação toroidal, ETRF, conforme o capítulo 4 (equação 4.68), temos

$$
\begin{array}{r}
\boldsymbol{v}_{\mathbf{0}}=\omega_{0} R \hat{\boldsymbol{e}}_{\perp}+u_{0} R_{0} \hat{\boldsymbol{e}}_{\|}, \\
\omega_{0}=\frac{\varepsilon}{q} \frac{R_{0}}{R} u_{0} \quad \text { e } \quad u_{0}=\frac{R}{R_{0}} M_{T} \omega_{s 0}, \\
\frac{\partial \rho_{0}}{\partial \theta}=2 \gamma M_{T}^{2} \frac{R}{R_{0}} \rho_{0} \varepsilon \sin \theta \quad \text { e } \quad \frac{\partial p_{0}}{\partial \theta}=\frac{\omega_{s 0}^{2} R_{0}^{2}}{\gamma} \frac{\partial \rho_{0}}{\partial \theta}
\end{array}
$$

de forma que, utilizando 6.32 e 6.33, podemos calcular as grandezas dadas em 6.6 e obter

$$
\begin{array}{r}
\bar{F}_{\perp 0} \approx-M_{T}\left(\bar{u}_{1 s}+\frac{M_{T}}{2} \bar{\rho}_{1 s}\right), \quad \bar{F}_{\perp c}=\bar{F}_{\| c}=0, \quad \bar{F}_{\| s} \approx 2 \delta M_{T}, \\
\bar{G}_{s} \approx-2 \gamma M_{T}^{2} \sin \theta, \quad \bar{G}_{c}=0 \\
\bar{H}_{s} \approx-2 M_{T}^{2} \sin \theta, \quad \bar{H}_{c}=0,
\end{array}
$$

sendo que os cálculos detalhados desta substituição são apresentados no apêndice D (equação D.32). A constante $\delta=1$ foi introduzida para contestar o resultado obtido em [29], que considera $\delta=1 / 2$ em seus cálculos. 
Sendo assim, as equações 6.22 a 6.28 ficam

$$
\begin{gathered}
-i \Omega-M_{T} \bar{u}_{1 s}-\frac{M_{T}^{2}}{2} \bar{\rho}_{1 s}-p_{1 s}=0 \\
\bar{u}_{1 s}=-\frac{i}{\Omega}\left(2 \delta M_{T}-\frac{\bar{p}_{1 c}}{q}\right), \\
\bar{u}_{1 c}=-\frac{i}{\Omega} \frac{\bar{p}_{1 s}}{q}, \\
\bar{\rho}_{1 s}=-\frac{i}{\Omega}\left[2\left(1-\gamma M_{T}^{2}\right)-\frac{\bar{u}_{1 c}}{q}\right] \\
\bar{p}_{1 c}=-\frac{i}{\Omega} \frac{\bar{u}_{1 s}}{q} . \\
\bar{p}_{1 s}=-\frac{i}{\Omega} \frac{\bar{u}_{1 s}}{q}, \\
\left.\Omega\left[1-M_{T}^{2}\right)-\frac{\bar{u}_{1 c}}{q}\right] \mathrm{e}
\end{gathered}
$$

Substituindo $\bar{p}_{1 s}$ de 6.48 e $\bar{p}_{1 c}$ de 6.49 , respectivamente em 6.45 e 6.44 , obtém-se $\bar{u}_{1}$,

$$
\begin{array}{r}
\bar{u}_{1}=\bar{u}_{1 s} \sin \theta+\bar{u}_{1 c} \cos \theta \\
\bar{u}_{1 s}=i \frac{2 \delta M_{T} q^{2} \Omega}{1-q^{2} \Omega^{2}} \quad \text { e } \quad \bar{u}_{1 c}=\frac{2\left(1-M_{T}^{2}\right) q}{1-q^{2} \Omega^{2}} .
\end{array}
$$

A partir da substituição de $\bar{u}_{1}$ em 6.46-6.49, obtêm-se $\bar{\rho}_{1}$ e $\bar{p}_{1}$, 


$$
\begin{gathered}
\bar{\rho}_{1}=\bar{\rho}_{1 s} \sin \theta+\bar{\rho}_{1 c} \cos \theta \\
\bar{\rho}_{1 s}=\frac{2 i(1-\gamma)\left(q^{2} \Omega^{2}-M_{T}^{2}\right)}{\Omega\left(1-q^{2} \Omega^{2}\right)}, \quad \rho_{1 c}=\frac{2 \delta M_{T} q}{1-q^{2} \Omega^{2}}, \quad \mathrm{e} \\
\bar{p}_{1}=\bar{p}_{1 s} \sin \theta+\bar{p}_{1 c} \cos \theta \\
\bar{p}_{1 s}=i \frac{2 q^{2} \Omega\left(1-M_{T}^{2}\right)}{1-q^{2} \Omega^{2}}, \quad \bar{p}_{1 c}=\frac{2 \delta M_{T} q}{1-q^{2} \Omega^{2}} .
\end{gathered}
$$

Ao inserir $\bar{u}_{1 s}, \bar{\rho}_{1 s}$ e $\bar{p}_{1 s}$ em 6.43 , obtemos a equação para $\Omega$,

$$
\Omega\left(1+\frac{2 \delta q^{2} M_{T}^{2}}{1-q^{2} \Omega^{2}}+\frac{2 q^{2}\left(1-M_{T}^{2}\right)}{1-q^{2} \Omega^{2}}\right)+\frac{1}{\Omega} \frac{(1-\gamma)\left(q^{2} \Omega^{2}-M_{T}^{2}\right) M_{T}^{2}}{1-q^{2} \Omega^{2}}=0,
$$

que pode ser escrita de forma mais simplificada como

$$
\frac{\Omega^{4}-2 f_{0, \mathrm{tr}} \Omega^{2}-f_{1, \mathrm{tr}}}{\Omega\left(1-q^{2} \Omega^{2}\right)}=0
$$

onde

$$
f_{0, \mathrm{tr}}=1+\frac{1}{2 q^{2}}+\left(\delta-\frac{1}{2}-\frac{\gamma}{2}\right) M_{T}^{2} \quad \text { e } \quad f_{1, \mathrm{tr}}=\frac{(\gamma-1)}{q^{2}} M_{T}^{4}
$$

e cujas soluções são

$$
\Omega_{z, \mathrm{tr}}^{e}=-i \Gamma_{z, \mathrm{tr}}, \quad \Omega_{z, \mathrm{tr}}^{i}=i \Gamma_{z, \mathrm{tr}} \quad \text { e } \quad \Omega_{g, \mathrm{tr}}=f_{g, \mathrm{tr}}
$$

em que

$$
\Gamma_{z, \mathrm{tr}}=\left(\sqrt{f_{0, \mathrm{tr}}^{2}+f_{1, \mathrm{tr}}}-f_{0, \mathrm{tr}}\right)^{1 / 2} \text { e } f_{g, \mathrm{tr}}=\left(f_{0, \mathrm{tr}}+\sqrt{f_{0, \mathrm{tr}}^{2}+f_{1, \mathrm{tr}}}\right)^{1 / 2} .
$$

Podemos notar que, se $\delta=1 / 2$ em 6.54, teríamos o resultado obtido por [29], com exceção do termo $M_{T}^{2}$ ao invés de $M_{T}^{4}$ em $f_{0, \text { tr }}$, entretanto, consideramos que tal resultado é incorreto, uma vez que, nos nossos cálculos, $\delta=1$. Atribuímos o erro do artigo ao cálculo do termo $\left(\boldsymbol{u}_{0} \cdot \boldsymbol{\nabla}\right) \boldsymbol{v}+(\boldsymbol{v} \cdot \boldsymbol{\nabla}) \boldsymbol{u}_{0}$, em que $\boldsymbol{u}_{0}$ representa a velocidade de equilíbrio e $\boldsymbol{v}$ a velocidade perturbada. Além disso, o artigo nos diz que há ondas de som se propagando 
no plasma, ou seja, $\Omega=\frac{1}{q}$ seria solução de 6.53, o que é facilmente identificável como incorreto, pois, basta verificar que tal valor anula o denominador de $6.53 \mathrm{e}$, portanto, não é uma solução aceitável. De fato, o termo $1 / q^{2}$ aparece na solução, entretanto a curvatura geodésica do campo magnético faz com que as frequências dos ZF e GAM sejam alteradas em relação à frequência de ondas acústicas $\left(1 / q^{2}\right)$.

Sendo assim, as soluções que obtivemos são dadas por 6.55 e 6.56, onde

$$
f_{0, \mathrm{tr}}=1+\frac{1}{2 q^{2}}+\frac{1-\gamma}{2} M_{T}^{2} \quad \text { e } \quad f_{1, \mathrm{tr}}=\frac{(\gamma-1)}{q^{2}} M_{T}^{4}
$$

No limite $\gamma \rightarrow 1$, ou seja, no limite de condutividade de calor infinita, ou temperatura constante, a rotação toroidal não influencia os ZF e GAM, ou seja, neste limite, de acordo com $6.55,6.56,6.57$ e as soluções de 6.40 ,

$$
\Omega_{z, \mathrm{tr}}(\gamma \rightarrow 1)=\Omega_{z, \mathrm{nr}}=0 \quad \text { e } \quad \Omega_{g, \mathrm{tr}}(\gamma \rightarrow 1)=\Omega_{g, \mathrm{nr}}=\sqrt{2+\frac{1}{q^{2}}}
$$

\subsection{Equilíbrio com rotação binormal - EBRF}

Para o equilíbrio com rotação binormal, ou rotação de deriva, temos, conforme o capítulo 4 (equação 4.69),

$$
\begin{gathered}
\boldsymbol{v}_{\mathbf{0}}=\omega_{0} R \hat{\boldsymbol{e}}_{\perp}, \quad \omega_{0}=M_{\perp} \omega_{s 0} \\
\frac{\partial \rho_{0}}{\partial \theta}=2 \alpha \frac{R_{0}}{R} \rho_{0} \varepsilon \sin \theta \quad \text { e } \quad \frac{\partial p_{0}}{\partial \theta}=2 \gamma \beta \frac{R_{0}}{R} p_{0} \varepsilon \sin \theta=\frac{\beta}{\alpha} \omega_{s 0}^{2} R_{0}^{2} \frac{\partial \rho_{0}}{\partial \theta} .
\end{gathered}
$$

As grandezas adimensionais são dadas por

$$
\begin{array}{rr}
\bar{F}_{\| s}=M_{\perp}\left(2 \frac{\varepsilon}{q}+\frac{\bar{u}_{1 c}}{\varepsilon}\right), & \bar{F}_{\| c}=-\frac{M_{\perp}}{\varepsilon} \bar{u}_{1 s}, \\
\bar{F}_{\perp 0}=M_{\perp}\left(\frac{M_{\perp}}{2} \bar{\rho}_{1 s}-\frac{\varepsilon}{q} \bar{u}_{1 s}\right), & \bar{F}_{\perp c}=\frac{\varepsilon M_{\perp}^{2}}{2} \bar{\rho}_{1 s} \\
\bar{G}_{s}=\frac{M_{\perp}}{\varepsilon} \bar{\rho}_{1 c}-2 \alpha, & \bar{G}_{c}=-\frac{M_{\perp}}{\varepsilon} \bar{\rho}_{1 s} \\
\bar{H}_{s}=\frac{M_{\perp}}{\varepsilon} \bar{p}_{1 c}-2 \beta, & \bar{H}_{c}=-\frac{M_{\perp}}{\varepsilon} \bar{p}_{1 s},
\end{array}
$$


conforme 6.21 e a equação D.33 do apêndice D.

A substituição de 6.60 em $6.22-6.28$ nos permite obter o seguinte conjunto de equações:

$$
\begin{gathered}
-i \Omega+M_{\perp}\left(\frac{M_{\perp}}{2} \bar{\rho}_{1 s}-\frac{\varepsilon}{q} \bar{u}_{1 s}\right)-\bar{p}_{1 s}=0, \\
\bar{u}_{1 s}=\frac{-i}{\Omega}\left[M_{\perp}\left(2 \frac{\varepsilon}{q}+\frac{\bar{u}_{1 c}}{\varepsilon}\right)-\frac{\bar{p}_{1 c}}{q}\right], \\
\bar{u}_{1 c}=\frac{-i}{\Omega}\left(-\frac{M_{\perp}}{\varepsilon} \bar{u}_{1 s}+\frac{\bar{p}_{1 s}}{q}\right), \\
\bar{\rho}_{1 s}=\frac{-i}{\Omega}\left[2(1-\alpha)-\frac{\bar{u}_{1 c}}{q}+\frac{M_{\perp}}{\varepsilon} \bar{\rho}_{1 c}\right], \\
\bar{p}_{1 c}=\frac{-i}{\Omega}\left(\frac{\bar{u}_{1 s}}{q}-\frac{M_{\perp}}{\varepsilon} \bar{p}_{1 s}\right), \\
\bar{\rho}_{1 c}=\frac{-i}{\Omega}\left(\frac{\bar{u}_{1 s}}{q}-\frac{M_{\perp}}{\varepsilon} \bar{\rho}_{1 s}\right), \\
\frac{-i}{\Omega}\left[\left(2(1-\beta)-\frac{\bar{u}_{1 c}}{q}\right)+\frac{M_{\perp}}{\varepsilon} \bar{p}_{1 c}\right] \mathrm{e}
\end{gathered}
$$

que podem ser escritas na forma matricial, 


$$
\left(\begin{array}{ccccccc}
-i \Omega & -1 & 0 & \frac{M_{\perp}^{2}}{2} & 0 & -\frac{\varepsilon}{q} M_{\perp} & 0 \\
2 \frac{\varepsilon}{q} M_{\perp} & 0 & -\frac{1}{q} & 0 & 0 & -i \Omega & \frac{M_{\perp}}{\varepsilon} \\
0 & \frac{1}{q} & 0 & 0 & 0 & \frac{-M_{\perp}}{\varepsilon} & -i \Omega \\
2(1-\alpha) & 0 & 0 & -i \Omega & \frac{M_{\perp}}{\varepsilon} & 0 & -\frac{1}{q} \\
0 & 0 & 0 & \frac{-M_{\perp}}{\varepsilon} & -i \Omega & \frac{1}{q} & 0 \\
2(1-\beta) & -i \Omega & \frac{M_{\perp}}{\varepsilon} & 0 & 0 & 0 & -\frac{1}{q} \\
0 & \frac{-M_{\perp}}{\varepsilon} & -i \Omega & 0 & 0 & \frac{1}{q} & 0
\end{array}\right)\left(\begin{array}{c}
1 \\
\bar{p}_{1 s} \\
\bar{p}_{1 c} \\
\bar{\rho}_{1 s} \\
\bar{\rho}_{1 c} \\
\bar{u}_{1 s} \\
\bar{u}_{1 c}
\end{array}\right)=\left(\begin{array}{c}
0 \\
0 \\
0 \\
0 \\
0 \\
0 \\
0
\end{array}\right) .
$$

A equação 6.68 é uma equação do tipo $\hat{M} \cdot \hat{V}=\hat{0}$, onde $\hat{M}$ e $\hat{V}$ são matrizes não nulas correspondentes, respectivamente, às duas primeiras matrizes do lado esquerdo de 6.68, e $\hat{0}$ a matriz nula correspondente ao lado direito de 6.68. Para que não tenhamos a soluções trivial, ou seja, $\hat{V} \neq \hat{0}$, temos que impor a condição de que o determinante de $\hat{M}$ se anule. Tal imposição fornece uma equação para $\Omega$.

No apêndice $\mathrm{E}$, obtemos a equação decorrente $\operatorname{de} \operatorname{det} \hat{M}=0$ e a resolvemos para encontrar as soluções de $\Omega$. Ao supor $M_{\perp} \sim 1$, obtemos soluções da forma $\Omega \sim 1 / \varepsilon$, que não são consideradas aceitáveis partindo deste modelo, visto que, de acordo com o capítulo 3, tais frequências de alto valor invalidam a aproximação de quasi-neutralidade. Entretanto, ao supor o regime de baixa rotação de equilíbrio, considerando $M_{\perp}=\varepsilon m_{\perp}$, onde $m_{\perp} \sim 1$, encontramos soluções da forma $\Omega \sim 1$, as quais correspondem à frequência dos ZF e GAM. Tais soluções obtidas são dadas por:

$$
\Omega=m_{\perp}, \quad \Omega=\left(f_{0, \mathrm{br}}+f_{1, \mathrm{br}}\right)^{1 / 2} \text { e } \Omega=\left(f_{0, \mathrm{br}}-f_{1, \mathrm{br}}\right)^{1 / 2},
$$

onde 
$f_{0, \mathrm{br}}=\left(1-\beta+m_{\perp}^{2}\right)+\frac{1}{q^{2}}, \quad f_{1, \mathrm{br}}=\sqrt{(1-\beta)^{2}+\frac{4 m_{\perp}^{2}}{q^{2}}}$ e $\beta=\left\{\begin{array}{cc}0 & \text { no limite ENR } \\ 1 & \text { com rotação }\end{array}(6.76\right.$

Pelas equações 6.69 e 6.70, observa-se que, no limite sem rotação, ou seja, $\beta=0$ e $m_{\perp}=0$, obtemos o resultado antigo, $\Omega=0$ e $\Omega=f_{g, \mathrm{nr}}$, que corresponde à frequência dos ZF e GAM sem rotação.

Ao tomar $\beta=1$ e $m_{\perp} \neq 0$, a frequência dos ZF é

$$
\Omega=m_{\perp}
$$

ou seja, no equilíbrio com rotação binormal os ZF apresentam uma frequência não nula, o que atribuímos ao efeito doppler. No referencial do laboratório, devido à rotação de equilíbrio, observa-se que o campo elétrico responsável pela formação de ZF oscila com frequência $\omega=m_{\perp} \omega_{s 0}$. Trata-se de um novo resultado que só é observado para este tipo de rotação.

A frequência dos GAM são dadas por

$$
\Omega=m_{\perp}+\frac{1}{q} \quad \text { e } \quad \Omega=m_{\perp}-\frac{1}{q}
$$

de forma que, para $m_{\perp}=1 / q$, temos $\Omega=0$ e $\Omega=2 / q$. Para $m_{\perp}=2 / q$, as frequências são $\Omega=1 / q$, que corresponde à frequência de ondas de som; e $\Omega=3 / q$, que é o triplo da frequência das ondas de som.

Na equação 6.72 nota-se que que ocorre o efeito doppler, visto que $m_{\perp}$ está relacionado com a frequência de rotação e $\frac{1}{q}$ com a frequência de ondas acústicas em plasmas de tokamak.

Mesmo para pequenas rotações de equilíbrio, $M_{\perp} \sim \varepsilon$, as frequências dos GAM são consideravelmente alteradas. Trata-se de um resultado novo que nos permite concluir que, para certos valores de rotação de equilíbrio, o efeito da curvatura geodésica pode ser anulado $(\Omega \propto 1 / q)$. É possível, inclusive, obter a frequência nula $\Omega=0$ para os GAM. Tais resultados poderão ser explorados no futuro com o intuito de reduzir o transporte turbulento. 


\section{Capítulo 7}

\section{Conclusões e propostas para trabalhos futuros}

Neste trabalho, utilizamos as equações da MHD ideal perturbadas até primeira ordem com relação ao tempo para derivar a frequência de oscilação dos fluxos zonais (ZF) e modos acústicos geodésicos (GAM) em tokamaks de secção circular com alta razão de aspecto. Como tais equações, mesmo perturbadas, dependem de grandezas do equilíbrio, foi necessário estudar o equilíbrio em tais tokamaks.

Fizemos isso considerando, inicialmente, plasmas sem rotação (ENR) e, posteriormente, plasmas com rotação (ER) toroidal (ETRF) e binormal (EBRF). O problema de ENR para tokamaks de alta razão de aspecto possui solução analítica relativamente simples na literatura, o que não ocorre com o problema de ER, em que é necessário considerar casos especiais para encontrar uma solução analítica. O nosso objetivo, ao estudar o equilíbrio, foi encontrar a dependência com o ângulo poloidal de tais grandezas de equilíbrio, pois tal dependência é o que diferencia o equilíbrio em sistemas cilíndricos do equilíbrio em sistemas toroidais, como é o caso de tokamaks. Constatamos que a curvatura geodésica do tokamak e, consequentemente, do campo magnético de equilíbrio deste, desempenha um papel fundamental no surgimento dos GAM e dos ZF.

Os GAM são similares às ondas de som em plasmas, com a diferença de que, em sistemas toroidais, o plasma, mesmo estudado como um fluido neutro, interage com o campo magnético devido à curvatura geodésica destes, ou mais precisamente, devido à dependência com o ângulo poloidal destes, o que na literatura é referido como o acoplamento do modo $m=0$ do campo elétrico com o modo $m=1$ da densidade (pressão), ambos perturbados. Este acoplamento é causado pela quebra da simetria poloidal, o que ocorre em sistemas toroidais, mas não em sistemas cilíndricos. Os GAM surgem devido a duas características essenciais: a perturbação da velocidade é compressível, o que já era 
esperado, assim como ocorre na propagação de ondas de som; e as superfícies magnéticas são eletricamente neutras. Embora haja fluxos de corrente elétrica através das superfícies magnéticas, do contrário não existiria um campo elétrico oscilante, tais fluxos variam de direção e de intensidade com a posição de forma que a média ao longo de uma superfície magnética desses fluxos é nula. Em outras palavras, ao considerar uma superfície em sua totalidade, o fluxo resultante que a atravessa é nulo e portanto a superfície magnética é eletricamente neutra.

Em contrapartida, nos ZF, a velocidade perturbada é compressível e, embora haja um campo elétrico perturbado, este não oscila com o tempo, visto que a frequência de oscilação obtida é nula. Neste caso, não há perturbação temporal da densidade (pressão) e, como consequência, não há fluxo de corrente entre as superfícies magnéticas (nem mesmo localmente). O campo elétrico inicial gera um fluxo binormal incompressível, o qual é compensado por um fluxo paralelo (ao longo das linhas de campo magnético) que, juntamente com o fluxo inicial, faz com que o fluxo total seja compressível. Estes dois fluxos, por outro lado, são o que mantém o campo elétrico perturbado sem que sejam necessários fluxos de correntes através das superfícies magnéticas.

Nos parágrafos anteriores, não levamos em conta o efeito que a rotação do plasma no equilíbrio causa nos GAM e nos ZF. No caso de ETRF, com exceção do regime de condutividade de calor infinita, em que as frequências dos GAM e ZF não se alteram pela presença da rotação, os ZF tornam-se instáveis e os GAM têm sua frequência de oscilação modificada. Obtivemos um resultado diferente para a frequência dos GAM e ZF da referência considerada [29]. Embora discordemos de tal referência nos resultados da frequência dos ZF e GAM e na afirmação de que ondas de som se propagam no plasma com ETRF, não negamos a sua importância para este trabalho.

No regime de condutividade de calor finita, há duas possibilidades para os ZF: a primeira é a de que haja um crescimento exponencial das grandezas perturbadas com o tempo (instabilidade), de forma que termos não lineares das equações da MHD perturbadas (termos de segunda ordem e ordens superiores) teriam que ser considerados no modelo; a segunda possibilidade é a de que os ZF decaiam exponencialmente com o tempo e, neste caso, em um curto intervalo de tempo eles se extinguiriam.

Ao nosso ver, a presença da rotação toroidal influencia negativamente na supressão de turbulência e transporte, pelo menos no processo de interação da turbulência com os $\mathrm{ZF}$, visto que os $\mathrm{ZF}$, ao se tornarem instáveis, poderiam saturar rapidamente e, ao se extinguirem rapidamente, teriam seu tempo de interação com a turbulência muito limitado. Para minimizar este efeito negativo da rotação toroidal, mecanismos capazes de aumentar a condutividade térmica do plasma poderiam ser estudados. A obtenção de 
regimes de alta condutividade, provavelmente, impactaria positivamente na supressão de turbulência e transporte.

Com relação ao efeito do EBRF nos ZF e GAM, obtivemos uma frequência não nula para os ZF que depende linearmente da intensidade da rotação de equilíbrio. Já os GAM podem ter sua frequência aumentada ou diminuída e, inclusive, podem ter frequências nula quando a rotação de equilíbrio atingir um determinado valor; tal valor corresponde ao inverso da multiplicação da razão de aspecto pelo fator de segurança. Embora nenhuma instabilidade tenha sido encontrada, nossos resultados permitiriam, em princípio, determinar a geometria do campo magnético determinando o fator de qualidade experimental a partir de medições das frequências dos GAM e ZF em tokamaks de secção circular com alta razão de aspecto.

Ademais enfatizamos que até mesmo pequenas rotações, causadas pelo campo elétrico, são capazes de mudar drasticamente a frequência dos GAM e ZF, e isto se deve ao impacto do efeito toroidal na variação da pressão de equilíbrio. Consideramos, no entanto, que não há fontes ou sorvedouros no plasma, de forma que, ao tratarmos de plasmas reais, com fluxos externos, os resultados poderão ser diferentes.

Não poderíamos deixar de citar a necessidade de desenvolver trabalhos sobre a saturação dos ZF instáveis; para isso, teríamos que considerar efeitos não lineares, o que provavelmente requer um tratamento estatístico ou caótico dos ZF. Tal tratamento deve ser feito a partir da teoria cinética, em que outros efeitos teriam que ser levados em conta, tal como o amortecimento de Landau.

Estudos sobre a interação dos ZF e GAM com a turbulência e o impacto no transporte anômalo são de extrema importância no desenvolvimento de reatores a fusão. Trabalhos sobre isso poderiam se beneficiar desta dissertação, pelo fato de esta levar em conta o efeito da rotação de equilíbrio poloidal e toroidal nos ZF e GAM, rotação que provavelmente estará presente nos futuros reatores a fusão.

A solução do problema de equilíbrio com rotação, seja ela causada por injeção de partículas neutras, por difusão ambipolar de partículas carregadas, ou até mesmo por outros tipos de rotação não descritos aqui, certamente acrescentará novas ideias para futuros trabalhos em ZF e GAM e até mesmo para este trabalho. Sendo assim, não podemos deixar de enfatizar a necessidade de explorar mais a fundo o problema de equilíbrio com rotação, inclusive para tokamaks de secção não-circular e/ou com baixa razão de aspecto, que provavelmente serão os futuros reatores a fusão.

Nesta dissertação, consideramos os ZF e GAM localizados na borda da coluna de plasma. Porém, em muitos tokamaks e provavelmente nos futuros reatores a fusão, tais modos ocorrem também no centro da coluna de plasma, em que a aproximação 
para baixos valores do parâmetro $\beta$ não é adequada devido à forte pressão hidrostática no centro da coluna de plasma. Neste regime, é necessário lidar com campo elétrico e magnético que variam no tempo, ao contrario do que consideramos neste trabalho. Sendo assim, estudos sobre ZF e GAM eletromagnéticos serão imprescindíveis na construção de futuros reatores a fusão, uma vez que, para conseguir regimes de baixos coeficientes de transporte, é necessário criar barreiras de transporte mais próximas do centro da coluna de plasma. 


\section{Referências Bibliográficas}

[1] Transaction of Fusion Sience and Technology, 53 (2008)

[2] World Population Prospects: the 1994 revision, United Nations Population Division, New York 1994.

[3] A. Grubler, A. McDonald, Global Energy Perspectives. IIASA and World Energy Council, Cambridge University press 1998.

[4] F. Joos, The Atmospheric Carbon Dioxide Pertubation, Europhysiucs News, $27,6,213-218(1996)$

[5] I. Cook et al., "Safety and Environmental Impact of Fusion" Report EFDA-S-RE-1, EUR(01) CCE-FU/FTC 8/5 (April 2001)

[6] Hogan, W. J., Editor:Energy from Inertial Fusion, International Atomic Energy Agenc, Vienna, Austria (1995).

[7] Wesson, J. Tokamkas, 2nd edition, Clarendon Press, Oxford, 1997.

[8] http://www.iter.org/

[9] J. P. Freidberg, Ideal Magnetohydrodynamics.

[10] J. P. H. Goedbloed. and S. Poedts, Principles of magnetohydrodynamics.

[11] Terry, P. W. Reviews of Modern Physics, 2000, Vol. 72, 109. 2000

[12] Balescu, R. Aspects of Anomalous Transport in Plasmas, 2005.

[13] Balescu, R. Transport Processes in Plasmas, Classical Transport, 1, 1988.

[14] Balescu, R. Transport Processes in Plasmas, Neoclassical Transport, 2, 1988.

[15] Turnbull, A.D. et al; Nuclear Fusion, 1998, 38, 1467 
[16] Winsor N., Johnson J. L., Dawson J. M. Physics of Fluids, 1968, 11:2448

[17] F. F. Chen, Introduction to plasma physics and controlled nuclear fusion.

[18] D. Bohm; The Characteristics of Eletric Discharges in Magnetic Fields, A. Guthrie and R. Wakerling (1949)

[19] F. Wagner et al; Physical Review Letters, 49, 1408 (1982)

[20] Y. Koide et al.; Physical Review Letters, 72, 3662 (1994)

[21] http://www.iter.org/a/index_nav_4.htm

[22] K. H. Burrell; Physics of Plasmas, 4, 1499 (1997)

[23] P H Diamond, S-I Itoh, K Itoh and T S Hahm, Plasma Phys. Control. Fusion 47, R35, 2005

[24] Z. Lin et al; Science 281, 1835 (1998).

[25] R. J. Goldston, P. H. Rutherford; Introduction to Plasma Physics

[26] A. Fujisawa et al.; Nuclear Fusion, 47, 5718 (2007)

[27] H. P. Zehrfeld and B. Green, Nuclear Fusion, 26, 230 (1972)

[28] S. Yoshikawa, Phys. Fluids, 17, 178 (1974).

[29] Shaojie Wang, Physical Review Letters 97, 085002, 2006.

[30] F.L. Hinton and S.K. Wong, Phys. Fluids 28, 3082 (1985)

[31] H. Gerhauser et al; J. Nuc. Mat., 313-316, 893 (2003)

[32] Discussão pessoal com Dr. Artour G. Elfimov. 


\section{Apêndice A}

\section{Identidades vetoriais}

Neste apêndice apresentamos as principais identidades vetoriais muito utilizada em física de plasmas, eletromagnetismo e mecânica dos fluidos.

Sejam $\boldsymbol{A}=\boldsymbol{A}(\boldsymbol{r}), \boldsymbol{B}=\boldsymbol{B}(\boldsymbol{r})$ e $\boldsymbol{C}=\boldsymbol{C}(\boldsymbol{r})$ vetores quaisquer, $f=f(\boldsymbol{r})$ e $g=$ $g(\boldsymbol{r})$ funções escalares arbitrárias e $\boldsymbol{r}=\left(q_{1}, q_{2}, q_{3}\right)$ o vetor posição em um sistema de coordenadas tridimensional arbitrário; então as seguintes relações são válidas:

$$
\begin{gathered}
(\boldsymbol{A} \times \boldsymbol{B}) \cdot \boldsymbol{C}=(\boldsymbol{B} \times \boldsymbol{C}) \cdot \boldsymbol{A}=(\boldsymbol{C} \times \boldsymbol{A}) \cdot \boldsymbol{B} \quad \mathrm{e} \\
\boldsymbol{A} \times(\boldsymbol{B} \times \boldsymbol{C})=(\boldsymbol{A} \cdot \boldsymbol{C}) \boldsymbol{B}-(\boldsymbol{A} \cdot \boldsymbol{B}) \boldsymbol{C}
\end{gathered}
$$

Utilizando A.1 e A.2, obtém-se

$$
\begin{aligned}
& (\boldsymbol{A} \times \boldsymbol{B}) \cdot(\boldsymbol{C} \times \boldsymbol{D})=(\boldsymbol{A} \cdot \boldsymbol{C})(\boldsymbol{B} \cdot \boldsymbol{D})-(\boldsymbol{A} \cdot \boldsymbol{D})(\boldsymbol{B} \cdot \boldsymbol{C}) \mathrm{e} \\
& (\boldsymbol{A} \times \boldsymbol{B}) \times(\boldsymbol{C} \times \boldsymbol{D})=[(\boldsymbol{A} \times \boldsymbol{B}) \cdot \boldsymbol{D}] \boldsymbol{C}-[(\boldsymbol{A} \times \boldsymbol{B}) \cdot \boldsymbol{C}] \boldsymbol{D} .
\end{aligned}
$$

É possível mostrar também as seguintes relações envolvendo operadores vetoriais:

$$
\boldsymbol{\nabla} \cdot(\boldsymbol{\nabla} \times \boldsymbol{A})=0 \quad \text { então se } \quad \boldsymbol{\nabla} \cdot \boldsymbol{B}=0 \Longleftrightarrow \boldsymbol{B}=\boldsymbol{\nabla} \times \boldsymbol{A} \text {, }
$$




$$
\boldsymbol{\nabla} \times(\boldsymbol{\nabla} f)=0 \quad \text { então se } \boldsymbol{\nabla} \times \boldsymbol{B}=0 \Longleftrightarrow \boldsymbol{B}=\boldsymbol{\nabla} f
$$

em que a regra da cadeia também se verifica em

$$
\begin{aligned}
& \boldsymbol{\nabla}(f g)=f(\boldsymbol{\nabla} g)+g(\boldsymbol{\nabla} f) \\
& \boldsymbol{\nabla} \cdot(f \boldsymbol{A})=f \boldsymbol{\nabla} \cdot \boldsymbol{A}+\boldsymbol{A} \cdot \boldsymbol{\nabla} f \\
& \boldsymbol{\nabla} \times(f \boldsymbol{A})=f(\boldsymbol{\nabla} \times \boldsymbol{A})+\boldsymbol{\nabla} f \times \boldsymbol{A}, \\
& \nabla \cdot(\boldsymbol{A} \times \boldsymbol{B})=\boldsymbol{B} \cdot(\boldsymbol{\nabla} \times \boldsymbol{A})-\boldsymbol{A} \cdot(\boldsymbol{\nabla} \times \boldsymbol{B}), \\
& \boldsymbol{\nabla} \times(\boldsymbol{A} \times \boldsymbol{B})=\boldsymbol{A}(\boldsymbol{\nabla} \cdot \boldsymbol{B})-\boldsymbol{B}(\boldsymbol{\nabla} \cdot \boldsymbol{A})+(\boldsymbol{B} \cdot \boldsymbol{\nabla}) \boldsymbol{A}-(\boldsymbol{A} \cdot \boldsymbol{\nabla}) \boldsymbol{B}, \\
& \boldsymbol{A} \times(\boldsymbol{\nabla} \times \boldsymbol{B})=\boldsymbol{\nabla}(\boldsymbol{A} \cdot \boldsymbol{B})-(\boldsymbol{A} \cdot \boldsymbol{\nabla}) \boldsymbol{B}-(\boldsymbol{B} \cdot \boldsymbol{\nabla}) \boldsymbol{A}-\boldsymbol{B} \times(\boldsymbol{\nabla} \times \boldsymbol{A}) \quad \mathrm{e} \\
& \nabla \times(\nabla \times A)=\nabla(\nabla \cdot A)-\nabla^{2} \boldsymbol{A}
\end{aligned}
$$

Sendo $V$ o volume, $S$ a área de uma superfície que engloba o volume $V$ e $\boldsymbol{l}$ um vetor com dimensão de comprimento que representa o contorno da área $S$, então as seguintes identidades

$$
\int_{V}(\boldsymbol{\nabla} \cdot \boldsymbol{B}) d V=\oint_{S} \boldsymbol{B} \cdot d \boldsymbol{S}
$$




$$
\int_{S}(\boldsymbol{\nabla} \times \boldsymbol{B}) \cdot d \boldsymbol{S}=\oint_{l} \boldsymbol{B} \cdot d \boldsymbol{l}
$$

são válidas, sendo que a primeira é conhecida com lei de Gauss e a segunda como Teorema de Stokes. 


\section{Apêndice B}

\section{Sistemas de coordenadas}

Neste apêndice, descrevemos os principais operadores vetoriais, gradiente, divergente, rotacional e laplaciano para os sistemas de coordenadas cilíndricas globais, coordenadas pseudo-toroidais e coordenadas cilíndricas. Também descrevemos a relação entre os derivativos destes sistemas de coordenadas.

\section{B.1 Sistema de coordenada arbitrário}

Para um sistema de coordenadas arbitrário, cuja métrica seja definida por

$$
(d s)^{2}=\sum_{i=1}^{3} h_{i}^{2}\left(d q_{i}\right)^{2}
$$

o gradiente, o divergente, o rotacional e o laplaciano são calculados por

$$
\begin{gathered}
\nabla \Psi=\sum_{i=1}^{3} \frac{1}{h_{i}} \frac{\partial \Psi}{\partial q_{i}} \hat{e}_{i}, \\
\boldsymbol{\nabla} \cdot \boldsymbol{B}=\frac{1}{h_{1} h_{2} h_{3}} \sum_{i=1}^{3} \frac{\partial\left(h_{j} h_{k} B_{i}\right)}{\partial q_{i}} \\
\boldsymbol{\nabla} \times \boldsymbol{B}=\sum_{i=1}^{3} \frac{1}{h_{j} h_{k}}\left[\frac{\partial\left(h_{k} A_{k}\right)}{\partial q_{j}}-\frac{\partial\left(h_{j} A_{j}\right)}{\partial q_{k}}\right] \hat{e}_{i} \quad \mathrm{e}
\end{gathered}
$$




$$
\nabla^{2} \Psi=\frac{1}{h_{1} h_{2} h_{3}} \sum_{i=1}^{3} \frac{\partial}{\partial q_{i}}\left[\frac{h_{j} h_{k}}{h_{i}} \frac{\partial \Psi}{\partial q_{i}}\right]
$$

em que $i, j$ e $k$ devem ser interpretados em ordem cíclica e nesta ordem, isto é:

- se $i=1$, então $j=2$ e $k=3$,

- se $i=2$ então $j=3$ e $k=1$ e

- se $i=3$ então $j=1$ e $k=2$.

\section{B.2 Coordenadas cilíndricas globais}

Em coordenadas cilíndricas globais, $\left(q_{1}, q_{2}, q_{3}\right) \rightarrow(R, \varphi, Z)$, a métrica é dada por

$$
(d s)^{2}=(d R)^{2}+R^{2}(d \varphi)^{2}+(d Z)^{2}
$$

de forma que $h_{1}=h_{3}=1$ e $h_{2}=R$. Utilizando B.2-B.5, obtemos

$$
\begin{gathered}
\nabla \Psi=\frac{\partial \Psi}{\partial R} \hat{e}_{R}+\frac{1}{R} \frac{\partial \Psi}{\partial \varphi} \hat{e}_{\varphi}+\frac{\partial \Psi}{\partial Z} \hat{e}_{Z} \\
\nabla \cdot \boldsymbol{B}=\frac{1}{R} \frac{\partial\left(R B_{r}\right)}{\partial R}+\frac{1}{R} \frac{\partial B_{\varphi}}{\partial \varphi}+\frac{\partial B_{Z}}{\partial Z} \\
\nabla \times \boldsymbol{B}=\left[\frac{1}{R} \frac{\partial B_{Z}}{\partial \varphi}-\frac{\partial B_{\varphi}}{\partial Z}\right] \hat{e}_{R}+\left[\frac{\partial B_{R}}{\partial Z}-\frac{\partial B_{Z}}{\partial R}\right] \hat{e}_{\varphi}+\left[\frac{1}{R} \frac{\partial\left(R B_{\varphi}\right)}{\partial R}-\frac{1}{R} \frac{\partial B_{R}}{\partial \varphi}\right] \hat{e}_{Z} \\
\nabla^{2} \Psi=\frac{1}{R} \frac{\partial}{\partial R}\left(R \frac{\partial \Psi}{\partial R}\right)+\frac{1}{R^{2}} \frac{\partial^{2} \Psi}{\partial \varphi^{2}}+\frac{\partial^{2} \Psi}{\partial Z^{2}} .
\end{gathered}
$$

Para o mesmo cálculo em coordenadas cilíndricas locais, basta fazer as substituições $R \rightarrow r, \varphi \rightarrow \theta$ e $Z \rightarrow z$ nas expressões B.6-B.10. 


\section{B.3 Coordenadas pseudo-toroidais}

Em coordenadas pseudo-toroidais, $\left(q_{1}, q_{2}, q_{3}\right) \rightarrow(r, \theta, \phi)$, a métrica é dada por

$$
\begin{array}{r}
(d s)^{2}=(d r)^{2}+r^{2}(d \theta)^{2}+R^{2}(d \phi)^{2}, \quad \text { onde } \\
R=R_{0}(1+\varepsilon \cos \theta) \quad \text { e } \quad \varepsilon=\frac{r}{R_{0}}
\end{array}
$$

de forma que $h_{1}=1, h_{2}=r$ e $h_{3}=R$. Analogamente, utilizando B.2-B.5, obtém-se

$$
\begin{gathered}
\nabla \Psi=\frac{\partial \Psi}{\partial r} \hat{e}_{r}+\frac{1}{r} \frac{\partial \Psi}{\partial \theta} \hat{e}_{\theta}+\frac{1}{R} \frac{\partial \Psi}{\partial \phi} \hat{e}_{\phi} \\
\nabla \cdot \boldsymbol{B}=\frac{1}{R r}\left(\frac{\partial}{\partial r}\left(R r B_{r}\right)+\frac{\partial}{\partial \theta}\left(R B_{\theta}\right)+r \frac{\partial B_{\phi}}{\partial \phi}\right) \\
\nabla \times \boldsymbol{B}=\frac{1}{R}\left[\left(\frac{1}{r} \frac{\partial\left(R B_{\phi}\right)}{\partial \theta}-\frac{\partial B_{\theta}}{\partial \phi}\right) \hat{e}_{r}+\left(\frac{\partial B_{r}}{\partial \phi}-\frac{\partial\left(R B_{\phi}\right)}{\partial r}\right) \hat{e}_{\theta}+\frac{R}{r}\left(\frac{\partial\left(r B_{\theta}\right)}{\partial r}-\frac{\partial B_{r}}{\partial \theta}\right) \hat{e}_{\phi}\right] \\
\nabla^{2} \Psi=\frac{1}{R r}\left[\frac{\partial}{\partial r}\left(R r \frac{\partial \Psi}{\partial r}\right)+\frac{\partial}{\partial \theta}\left(\frac{R}{r} \frac{\partial \Psi}{\partial \theta}\right)+\frac{\partial}{\partial \phi}\left(\frac{r}{R} \frac{\partial \Psi}{\partial \phi}\right)\right]
\end{gathered}
$$

Em muitos problemas práticos com sistemas toroidais, é util estabelecer a relação entre derivativos em coordenadas cilíndricas e coordenadas pseudo-toroidais, a seguir descrevemos as principais relações.

\section{B.4 Coordenadas cilíndricas locais}

O sistema de coordenadas cilíndricas locais, $(r, \theta, z)$, é o caso limite em que $R_{0} \rightarrow \infty$, e vale a relação $d z=R d \phi$. Sendo assim a métrica é dada por

$$
(d s)^{2}=(d r)^{2}+r^{2}(d \theta)^{2}+(d z)^{2} .
$$


De acordo com B.16, $h_{1}=1, h_{2}=r$ e $h_{3}=1$ e utilizando B.2-B.5, podemos escrever

$$
\begin{gathered}
\nabla \Psi=\frac{\partial \Psi}{\partial r} \hat{e}_{r}+\frac{1}{r} \frac{\partial \Psi}{\partial \theta} \hat{e}_{\theta}+\frac{\partial \Psi}{\partial z} \hat{e}_{z} \\
\nabla \cdot \boldsymbol{B}=\frac{1}{r} \frac{\partial\left(r B_{r}\right)}{\partial r}+\frac{1}{r} \frac{\partial B_{\theta}}{\partial \theta}+\frac{\partial B_{z}}{\partial z} \\
\nabla \times \boldsymbol{B}=\left(\frac{1}{r} \frac{\partial B_{z}}{\partial \theta}-\frac{\partial B_{\theta}}{\partial z}\right) \hat{e}_{r}+\left(\frac{\partial B_{r}}{\partial z}-\frac{\partial B_{z}}{\partial r}\right) \hat{e}_{\theta}+\frac{1}{r}\left(\frac{\partial\left(r B_{\theta}\right)}{\partial r}-\frac{\partial B_{r}}{\partial \theta}\right) \hat{e}_{z} \quad \text { e } \\
\nabla^{2} \Psi=\frac{1}{r} \frac{\partial}{\partial r}\left(r \frac{\partial \Psi}{\partial r}\right)+\frac{1}{r^{2}} \frac{\partial^{2} \Psi}{\partial \theta^{2}}+\frac{\partial^{2} \Psi}{\partial z^{2}} .
\end{gathered}
$$

\section{B.5 Relação entre coordenadas cilíndricas e coordena- das pseudo-toroidais}

A relação entre $(R, \phi, Z)$ em coordenadas cilíndricas e $(r, \theta, \varphi)$ em coordenadas pseudotoroidais é dada por

$$
\begin{array}{r}
R=R_{0}+r \cos \theta \\
\phi=-\varphi \\
Z=r \sin \theta,
\end{array}
$$

Utilizando a regra da cadeia temos as seguintes relações

$$
\xi=\xi_{0}(R, Z) \rightarrow \xi_{1}[r(R, Z), \theta(R, Z)]
$$




$$
\begin{aligned}
& \frac{\partial \xi_{0}}{\partial R}=\frac{\partial r}{\partial R} \frac{\partial \xi_{1}}{\partial r}+\frac{\partial \theta}{\partial R} \frac{\partial \xi_{1}}{\partial \theta} \\
& \frac{\partial \xi_{0}}{\partial Z}=\frac{\partial r}{\partial Z} \frac{\partial \xi_{1}}{\partial r}+\frac{\partial \theta}{\partial Z} \frac{\partial \xi_{1}}{\partial \theta}
\end{aligned}
$$

Ao fazer $\xi_{0}=R$ e $\xi_{0}=Z$ em B.23 e utilizar B.21, obtém-se

$$
\frac{\partial r}{\partial R}=\cos \theta, \quad \frac{\partial \theta}{\partial R}=-\frac{\sin \theta}{r}, \quad \frac{\partial r}{\partial Z}=\sin \theta \quad \text { e } \quad \frac{\partial \theta}{\partial Z}=\frac{\cos \theta}{r}
$$

e, consequentemente, a relação entre operadores em coordenadas cilíndricas globais e operadores em coordenadas pseudo-toroidais,

$$
\begin{array}{r}
\frac{\partial}{\partial R}=\cos \theta \frac{\partial}{\partial r}-\frac{\sin \theta}{r} \frac{\partial}{\partial \theta} \\
\frac{\partial^{2}}{\partial R^{2}}=\cos ^{2} \theta \frac{\partial^{2}}{\partial r^{2}}+\frac{\sin ^{2} \theta}{r^{2}} \frac{\partial^{2}}{\partial \theta^{2}}+\frac{\sin ^{2} \theta}{r} \frac{\partial}{\partial r}+\frac{2 \sin \theta \cos \theta}{r^{2}} \frac{\partial}{\partial \theta}-\frac{2 \sin \theta \cos \theta}{r} \frac{\partial^{2}}{\partial r \partial \theta} \\
\frac{\partial}{\partial Z}=\sin \theta \frac{\partial}{\partial r}+\frac{\cos \theta}{r} \frac{\partial}{\partial \theta} \\
\frac{\partial^{2}}{\partial Z^{2}}=\sin ^{2} \theta \frac{\partial^{2}}{\partial r^{2}}+\frac{\cos ^{2} \theta}{r^{2}} \frac{\partial^{2}}{\partial \theta^{2}}+\frac{\cos ^{2} \theta}{r} \frac{\partial}{\partial r}-\frac{2 \sin \theta \cos \theta}{r^{2}} \frac{\partial}{\partial \theta}+\frac{2 \sin \theta \cos \theta}{r} \frac{\partial^{2}}{\partial r \partial \theta} .
\end{array}
$$

Finalmente, através de B.25, é possível converter o operador elíptico de um sistema de coordenadas para outro,

$$
\begin{array}{r}
\Delta^{*}=\frac{\partial^{2}}{\partial R^{2}}-\frac{1}{R} \frac{\partial}{\partial R}+\frac{\partial^{2}}{\partial Z^{2}} \rightarrow \Delta^{*}=\frac{\partial^{2}}{\partial r^{2}}+\frac{1}{r} \frac{\partial}{\partial r}+\frac{1}{r^{2}} \frac{\partial^{2}}{\partial \theta^{2}}-\frac{1}{R}\left(\cos \theta \frac{\partial}{\partial r}-\frac{\sin \theta}{r} \frac{\partial}{\partial \theta}\right) \quad \text { ou } \\
\Delta^{*}=\nabla^{2}-\frac{1}{R}\left(\cos \theta \frac{\partial}{\partial r}-\frac{\sin \theta}{r} \frac{\partial}{\partial \theta}\right),
\end{array}
$$

onde $\nabla^{2}=\frac{1}{r} \frac{\partial}{\partial r}\left(r \frac{\partial}{\partial r}\right)+\frac{1}{r^{2}} \frac{\partial^{2}}{\partial \theta^{2}}+\frac{\partial^{2}}{\partial z^{2}}$ é o laplaciano em coordenadas cilíndricas locais, B.20, considerando que há simetria em $z$, ou seja, $\frac{\partial^{2}}{\partial z^{2}}=0$.

\section{B.6 Derivativos de vetores}

A transformação dos versores de um sistema de coordenadas para outro é dada por 


$$
\begin{array}{r}
\hat{e}_{R}=\cos \theta \hat{e}_{r}-\sin \theta \hat{e}_{\theta} \\
\hat{e}_{\phi}=-\hat{e}_{\varphi} \\
\hat{e}_{Z}=\sin \theta \hat{e}_{r}+\cos \theta \hat{e}_{\theta} .
\end{array}
$$

Partindo do resultado conhecido de derivativos vetoriais em coordenadas cilíndricas,

$$
\begin{array}{r}
\frac{\partial \hat{e}_{r}}{\partial r}=\frac{\partial \hat{e}_{\theta}}{\partial r}=0, \quad \frac{\partial \hat{e}_{r}}{\partial \theta}=\hat{e}_{\theta} \quad \text { e } \quad \frac{\partial \hat{e}_{\theta}}{\partial \theta}=-\hat{e}_{r} \\
\frac{\partial \hat{e}_{R}}{\partial R}=\frac{\partial \hat{e}_{\varphi}}{\partial R}=-\frac{\partial \hat{e}_{\phi}}{\partial R}=0, \quad \frac{\partial \hat{e}_{R}}{\partial \varphi}=-\frac{\partial \hat{e}_{R}}{\partial \phi}=\hat{e}_{\varphi}=-\hat{e}_{\phi} \quad \text { e } \quad \frac{\partial \hat{e}_{\varphi}}{\partial \varphi}=\frac{\partial \hat{e}_{\phi}}{\partial \phi}=-\hat{e}_{R} \\
\frac{\partial \hat{e}_{Z}}{\partial R}=\frac{\partial \hat{e}_{Z}}{\partial \varphi}=-\frac{\partial \hat{e}_{Z}}{\partial \phi}=0
\end{array}
$$

e utilizando B.27, obtêm-se os derivativos vetoriais em coordenadas pseudo-toroidais,

$$
\begin{array}{r}
\frac{\partial \hat{e}_{r}}{\partial r}=0, \quad \frac{\partial \hat{e}_{\theta}}{\partial r}=0, \quad \frac{\partial \hat{e}_{\phi}}{\partial r}=0 \\
\frac{\partial \hat{e}_{r}}{\partial \theta}=\hat{e}_{\theta}, \quad \frac{\partial \hat{e}_{\theta}}{\partial \theta}=-\hat{e}_{r}, \quad \frac{\partial \hat{e}_{\phi}}{\partial \theta}=0 \\
\frac{\partial \hat{e}_{r}}{\partial \phi}=\cos \theta \hat{e}_{\phi}, \quad \frac{\partial \hat{e}_{\theta}}{\partial \phi}=-\sin \theta \hat{e}_{\phi}, \quad \frac{\partial \hat{e}_{\phi}}{\partial \phi}=-\cos \theta \hat{e}_{r}+\sin \theta \hat{e}_{\theta}
\end{array}
$$

Partindo de B.29, podemos obter para $B_{r}=0$ e $\frac{\partial B_{i}}{\partial \phi}=0$, com $i=\theta, \phi$, o seguinte resultado

$$
(\boldsymbol{B} \cdot \boldsymbol{\nabla}) \boldsymbol{B}=\left(-\frac{B_{\theta}^{2}}{r}-\frac{B_{\phi}^{2} \cos \theta}{R}\right) \hat{e}_{r}+\left(\frac{1}{2 r} \frac{\partial B_{\theta}^{2}}{\partial \theta}+\frac{B_{\phi}^{2} \sin \theta}{R}\right) \hat{e}_{\theta}+\left(\frac{B_{\theta}}{r} \frac{\partial B_{\phi}}{\partial \theta}-\frac{B_{\theta} B_{\phi} \sin \theta}{R}\right) \hat{e}_{\phi}
$$




\section{Apêndice C}

\section{Função de fluxo e resolução da equação de Grad-Shafranov}

Neste apêncie lidamos com a função $\Psi_{0}$, em duas dimensções e $\Psi$ em três dimensões. Provamos que podemos escrever o campo magnético em função destas duas funções e resolvemos a equação de Grad-Shafranov para encontrar a forma explicita de $\Psi$.

\section{C.1 Função $\Psi_{0}$ e o campo magnético $\boldsymbol{B}_{0}$ (uma dimen- são)}

Em coordenadas cilíndricas locais, $(r, \theta, z)$, com simetria em axial (em $z$ ), o campo magnético pode ser escrito como

$$
\boldsymbol{B}_{0}=\nabla \Psi_{0} \times \hat{e}_{z}+B_{z} \hat{e}_{z}
$$

Para provar tal afirmação, utilizam-se as relações A.7 e A.10 para escrever o $\boldsymbol{\nabla} \cdot \boldsymbol{B}_{0}$ como

$$
\boldsymbol{\nabla} \cdot \boldsymbol{B}_{0}=\hat{e}_{z} \cdot\left(\boldsymbol{\nabla} \times \boldsymbol{\nabla} \Psi_{0}\right)-\boldsymbol{\nabla} \Psi_{0} \cdot\left(\boldsymbol{\nabla} \times \hat{e}_{z}\right)+B_{z} \boldsymbol{\nabla} \cdot \hat{e}_{z}+\hat{e}_{z} \cdot \nabla B_{z}
$$

O primeiro termo do lado direito de C.2 é nulo naturalmente, de acordo com A.6; o divergente e o rotacional de $\hat{e}_{z}$ são dados por

$$
\boldsymbol{\nabla} \cdot \hat{e}_{z}=\frac{\partial 1}{\partial z}=0 \quad \text { e } \quad \boldsymbol{\nabla} \times \hat{e}_{z}=\frac{1}{r} \frac{\partial 1}{\partial \theta} \hat{e}_{r}-\frac{\partial 1}{\partial r} \hat{e}_{\theta}=0
$$


de acordo com B.16 e B.17. Sendo assim só resta provar que o termo $\hat{e}_{z} \cdot \nabla B_{z}$ é nulo. Tal termo é dado por

$$
\hat{e}_{z} \cdot \nabla B_{z}=\hat{e}_{z} \cdot\left(\hat{e}_{r} \frac{\partial B_{z}}{\partial r}+\hat{e}_{\theta} \frac{1}{r} \frac{\partial B_{z}}{\partial \theta}+\hat{e}_{z} \frac{\partial B_{z}}{\partial z}\right)=\frac{\partial B_{z}(r, \theta)}{\partial z}=0
$$

de acordo com B.15. Portanto C.1 fica provada.

\section{C.2 Função $\Psi$ e o campo magnético $B$ (duas dimen- sões)}

Em coordenadas pseudo-toroidais, $(r, \theta, \phi)$, com simetria azimutal (em $\phi)$, analogamente a C.1, o campo magnético pode ser escrito como

$$
\boldsymbol{B}=\frac{1}{R}\left(\nabla \Psi \times \hat{e}_{\phi}+F\right), \quad F=R B_{\phi},
$$

cuja demonstração de que tal função satisfaz $\boldsymbol{\nabla} \cdot \boldsymbol{B}=0$ segue abaixo:

$$
\boldsymbol{\nabla} \cdot \boldsymbol{B}=\frac{\hat{e}_{\phi}}{R} \cdot(\boldsymbol{\nabla} \times \boldsymbol{\nabla} \Psi)-\nabla \Psi \cdot\left(\boldsymbol{\nabla} \times \frac{\hat{e}_{\phi}}{R}\right)+F\left(\boldsymbol{\nabla} \cdot \hat{e}_{\phi}\right)+\hat{e}_{\phi} \cdot(\nabla F)
$$

De acordo com A.6, o primeiro termo do lado direito se anula. Utilizando B.13 e B.14, podemos escrever

$$
\boldsymbol{\nabla} \cdot \hat{e}_{\phi}=\frac{1}{R} \frac{\partial 1}{\partial \phi}=0 \quad \text { e } \quad \nabla \times\left(\frac{\hat{e}_{\phi}}{R}\right)=\frac{1}{R}\left[\frac{1}{r} \frac{\partial 1}{\partial \theta} \hat{e}_{r}-\frac{\partial 1}{\partial r} \hat{e}_{\theta}\right]=0
$$

e, utilizando B.12, o último termo do lado direito pode ser escrito como

$$
\hat{e}_{\phi} \cdot\left[\hat{e}_{r} \frac{\partial F(r, \theta)}{\partial r}+\frac{1}{r} \frac{\partial F(r, \theta)}{\partial \theta}+\hat{e}_{\phi} \frac{1}{R} \frac{\partial F(r, \theta)}{\partial \phi}\right]=0 .
$$

As equações C.6-C.8 provam C.5.

\section{C.3 Solução da equação de Grad-Shafranov}

A equação de Grad-Shafranov para o caso de equilíbrio estático é dada por 


$$
\Delta^{*} \Psi=-\mu_{0} R^{2} \frac{d p}{d \Psi}-\frac{1}{2} \frac{d F^{2}}{d \Psi}
$$

sendo que o operador elíptico $\Delta^{*}$, em coordenadas pseudo-toroidais, é dado por

$$
\Delta^{*}=\nabla^{2}-\frac{1}{R}\left(\cos \theta \frac{\partial}{\partial r}-\frac{\sin \theta}{r} \frac{\partial}{\partial \theta}\right)
$$

onde

$$
\nabla^{2}=\frac{\partial^{2}}{\partial r^{2}}+\frac{1}{r} \frac{\partial}{\partial r}+\frac{1}{r^{2}} \frac{\partial^{2}}{\partial \theta^{2}}
$$

de acordo com B.26.

Para a resolução de C.9, é conveniente escrevê-la na forma adimensional. Sendo assim, adotamos as seguintes convenções

$$
\begin{array}{r}
\Psi_{\text {norm }}=\frac{\Psi}{a R_{0} \bar{B}_{\theta}(a)} \\
r_{\text {norm }}=\frac{r}{a} \quad \text { e } \quad \epsilon=\frac{a}{R_{0}} \\
p_{\text {norm }}=\frac{p}{p_{\max }} \\
F_{\text {norm }}=\frac{F}{R_{0} B_{0}},
\end{array}
$$

onde

$$
\bar{B}_{\theta}(a)=\frac{\mu_{0} I_{P}}{2 \pi a}, \quad p_{\max }=p(r=0) \quad \text { e } \quad B_{0}=\frac{\mu_{0} N I_{T}}{2 \pi R_{0}}
$$

Ao substituir C.11 em C.9, obtém-se a equação de Grad-Shafranov adimensional,

$$
\nabla^{2} \Psi-\frac{\epsilon}{1+\epsilon r \cos \theta}\left(\cos \theta \frac{\partial \Psi}{\partial r}-\frac{\sin \theta}{r} \frac{\partial \Psi}{\partial \theta}\right)=-\frac{1}{2}\left[\beta_{\max }(1+\epsilon r \cos \theta)^{2} \frac{d p}{d \Psi}+\frac{B_{0}^{2}}{\bar{B}_{\theta}^{2}(a)} \frac{d F^{2}}{d \Psi}\right]
$$

na qual foi suprimido o índice "norm" para simplificar a notação e introduzida a grandeza adimensional $\beta_{\max }=2 \mu_{0} p_{\max } / \bar{B}_{\theta}^{2}(a)$, que representa o valor de $\beta$ considerando-se a máxima pressão e o campo magnético poloidal na borda do plasma. 
Para tokamkaks, consideram-se as seguintes ordens de grandeza:

$$
\epsilon \ll 1, \quad \frac{\bar{B}_{\theta}(a)}{B_{0}} \sim \epsilon, \quad \text { e } \quad \bar{q} \sim \frac{a B_{0}}{R_{0} \bar{B}_{\theta}(a)} \sim 1 .
$$

Sendo assim, separando C.13 em ordens de $\epsilon$, obtemos

$$
\epsilon^{0}\left[\nabla^{2} \Psi+J(\Psi)\right]+\epsilon^{1}\left[-\left(\cos \theta \frac{\partial \Psi}{\partial r}-\frac{\sin \theta}{r} \frac{\partial \Psi}{\partial \theta}\right)+\beta_{\max } r \cos \theta \frac{d p}{d \Psi}\right]+O\left(\epsilon^{2}\right)=0
$$

onde

$$
J(\Psi)=\frac{1}{2}\left(\beta_{\max } \frac{d p}{d \Psi}+\frac{B_{0}^{2}}{\bar{B}_{\theta}^{2}(a)} \frac{d F^{2}}{d \Psi}\right)
$$

A solução tentativa para tokamaks de secção circular, utilizando este modelo, é dada por

$$
\Psi(r, \theta)=\Psi_{0}(r)+\epsilon \Psi_{1}(r, \theta)+O\left(\epsilon^{2}\right)
$$

que, ao ser substituída em C.15 considerando apenas até primeira ordem em $\epsilon$, fornece

$\epsilon^{0}\left[\nabla^{2} \Psi_{0}+J\left(\Psi_{0}\right)\right]+\epsilon^{1}\left[\nabla^{2} \Psi_{1}+\frac{d J\left(\Psi_{0}\right)}{d \Psi_{0}} \Psi_{1}-\left(\cos \theta \frac{d \Psi_{0}}{d r}-\frac{\sin \theta}{r} \frac{\partial \Psi_{0}}{\partial \theta}\right)+\beta_{\max } r \cos \theta \frac{d p}{d \Psi_{0}}\right] \approx 0$

onde utilizamos a aproximação em série de Taylor

$$
J\left(\Psi_{0}+\epsilon \Psi_{1}\right)-J\left(\Psi_{0}\right) \approx \epsilon \frac{d J\left(\Psi_{0}\right)}{d \Psi_{0}} \Psi_{1}
$$

Ao impor a condição de que os coeficientes de $\epsilon^{0}$ e $\epsilon^{1}$ em C.18 se anulem, obtemos as seguintes equações a serem resolvidas

$$
\frac{d^{2} \Psi_{0}}{d r^{2}}+\frac{1}{r} \frac{d \Psi_{0}}{d r}+J\left(\Psi_{0}\right)=0 \quad \mathrm{e}
$$




$$
\begin{aligned}
& \frac{\partial^{2} \Psi_{1}}{\partial r^{2}}+\frac{1}{r} \frac{d \Psi_{1}}{d r}+\frac{1}{r^{2}} \frac{\partial^{2} \Psi_{1}}{\partial \theta^{2}}-\left(\frac{d \Psi_{0}}{d r}\right)^{-1} \Psi_{1} \frac{d}{d r}\left(\frac{d^{2} \Psi_{0}}{d r^{2}}+\frac{1}{r} \frac{d \Psi_{0}}{d r}\right)+ \\
& {\left[\beta_{\max }\left(\frac{d \Psi_{0}}{d r}\right)^{-1} r \frac{d p}{d r}-\frac{d \Psi_{0}}{d r}\right] \cos \theta=0 }
\end{aligned}
$$

onde utilizamos C.20 e a regra da cadeia para desenvolver C.21,

$$
\frac{d}{d \Psi_{0}}=\left(\frac{d \Psi_{0}}{d r}\right)^{-1} \frac{d}{d r}, \quad \frac{d J\left(\Psi_{0}\right)}{d \Psi_{0}}=-\left(\frac{d \Psi_{0}}{d r}\right)^{-1} \frac{d}{d r}\left(\frac{d^{2} \Psi_{0}}{d r^{2}}+\frac{1}{r} \frac{d \Psi_{0}}{d r}\right)
$$

A dependência em $\cos \theta$ de C.21 sugere a solução tentativa $\Psi_{1}=U(r) \cos \theta$, que, ao ser substituída em C.21, resulta em

$\frac{d^{2} U}{d r^{2}}+\frac{1}{r} \frac{d U}{d r}-\frac{U}{r^{2}}-\left(\frac{d \Psi_{0}}{d r}\right)^{-1} U \frac{d}{d r}\left(\frac{d^{2} \Psi_{0}}{d r^{2}}+\frac{1}{r} \frac{d \Psi_{0}}{d r}\right)+\beta_{\max }\left(\frac{d \Psi_{0}}{d r}\right)^{-1} r \frac{d p}{d r}-\frac{d \Psi_{0}}{d r}=0$

que também pode ser expressa como

$$
\frac{d \Psi_{0}}{d r}\left(\frac{d^{2} U}{d r^{2}}+\frac{1}{r} \frac{d U}{d r}-\frac{U}{r^{2}}\right)-\frac{d}{d r}\left(\frac{d^{2} \Psi_{0}}{d r^{2}}+\frac{1}{r} \frac{d \Psi_{0}}{d r}\right) U=0+S_{p}
$$

onde

$$
S_{p}=\left(\frac{d \Psi_{0}}{d r}\right)^{2}-r \beta_{\max } \frac{d p}{d r}
$$

é a solução particular de C.24.

É interessante notar que $U_{h}(r)=\frac{d \Psi_{0}}{d r}$ (o índice "h" especifica a solução homogênea) é solução de

$$
\frac{d \Psi_{0}}{d r}\left(\frac{d^{2} U_{h}}{d r^{2}}+\frac{1}{r} \frac{d U_{h}}{d r}-\frac{U_{h}}{r^{2}}\right)-\frac{d}{d r}\left(\frac{d^{2} \Psi_{0}}{d r^{2}}+\frac{1}{r} \frac{d \Psi_{0}}{d r}\right) U_{h}=0
$$

o que nos induz a verificar a solução tentativa 


$$
U_{p}(r)=f(r) U_{h}
$$

cujas relações

$$
\begin{array}{r}
\frac{d U_{p}}{d r}=f \frac{d U_{h}}{d r}+\frac{d f}{d r} U_{h} \\
\frac{d^{2} U_{p}}{d r^{2}}=f \frac{d^{2} U_{h}}{d r^{2}}+2 \frac{d f}{d r} \frac{d U_{h}}{d r}+\frac{d^{2} f}{d r^{2}} U_{h}
\end{array}
$$

serão úteis na substituição de C.27 em C.24.

Tal substituição resulta em

$$
\begin{array}{r}
U_{h}^{2} \frac{d^{2} f}{d r^{2}}+\frac{d f}{d r} \frac{d U_{h}^{2}}{d r}+\frac{U_{h}^{2}}{r} \frac{d f}{d r}=U_{h}^{2}-r \beta_{\max } \frac{d p}{d r} \quad \text { ou } \\
\frac{1}{r} \frac{d}{d r}\left(r U_{h}^{2} \frac{d f}{d r}\right)=U_{h}^{2}-r \beta_{\max } \frac{d p}{d r} \Longrightarrow r U_{h}^{2} \frac{d f}{d r}=\int_{0}^{r} d r^{\prime}\left(r^{\prime} U_{h}^{2}-r^{\prime 2} \beta_{\max } \frac{d p}{d r^{\prime}}\right) \Longrightarrow \\
f(r)=\int_{0}^{r} d r^{\prime} \frac{1}{r U_{h}^{2}} \int_{0}^{r} d r^{\prime \prime}\left(r^{\prime \prime} U_{h}^{2}-r^{\prime \prime 2} \beta_{\max } \frac{d p}{d r^{\prime}}\right) .
\end{array}
$$

Desta forma $U(r)=A U_{h}(r)+B U_{p}(r)$ (note que há duas constantes arbitrárias, $A$ e $B$. Podemos adotar $B=1$ e $A=C$ ) e, portanto, a solução geral é dada por

$$
\begin{gathered}
\Psi(r, \theta)=\Psi_{0}+\epsilon U_{h}\left[C+\int_{0}^{r} d r^{\prime} \frac{1}{r U_{h}^{2}} \int_{0}^{r} d r^{\prime \prime}\left(r^{\prime \prime} U_{h}^{2}-r^{\prime \prime 2} \beta_{\max } \frac{d p}{d r^{\prime}}\right)\right] \cos \theta, \quad \text { onde } \\
U_{h}=U_{h}(r)=\frac{d \Psi_{0}}{d r}
\end{gathered}
$$

A constante $C$ é determinada pelas condições de contorno para $\Psi(r, \theta)$. Ao supor $\Psi(a, \theta)=$ const., obtemos

$$
C=\int_{0}^{a} d r^{\prime} \frac{1}{r^{\prime} B_{\theta 0}^{2}} \int_{0}^{r^{\prime}} d r^{\prime \prime}\left(2 \mu_{0} r^{\prime \prime} \frac{d p}{d r^{\prime \prime}}-r^{\prime \prime} B_{\theta 0}^{2}\right) .
$$

Para determinar $\Psi_{0}(r)$, é necessário resolver a equação C.20 e, para isso, é interessante lembrar que 


$$
\boldsymbol{B}=\frac{1}{R}\left(\nabla \Psi \times \hat{e}_{\phi}+F \hat{e}_{\phi}\right)
$$

de acordo com C.5. É preciso, também, utilizar o campo magnético poloidal adimensional,

$$
B_{\theta_{\text {norm }}}=\frac{B_{\theta}}{\bar{B}_{\theta}(a)}=\left(\frac{1}{1+\epsilon r \cos \theta} \frac{\partial \Psi}{\partial r}\right)_{\text {norm }}
$$

que, em ordem $\epsilon^{0}$, é dado por

$$
B_{\theta 0}=\frac{d \Psi_{0}}{d r}=U_{h}(r)
$$

onde suprimimos o índice "norm" novamente para simplificar a notação.

Utilizando C.33, podemos escrever C.20 como

$$
\begin{aligned}
\frac{d B_{\theta 0}}{d r}+\frac{B_{\theta 0}}{r}+\frac{1}{2} \frac{1}{B_{\theta 0}} \frac{d}{d r}\left(\beta_{\max } p+\frac{B_{0}^{2}}{\bar{B}_{\theta}^{2}(a)} F^{2}\right) & =0 \Longrightarrow \\
\frac{d}{d r}\left(\beta_{\max } p+B_{\theta 0}^{2}+\frac{B_{0}^{2}}{\bar{B}_{\theta}^{2}(a)} F^{2}\right) & =-\frac{2 B_{\theta 0}^{2}}{r},
\end{aligned}
$$

que, nas variáveis físicas dimensionais, C.11, fica

$$
\frac{d}{d r}\left(p+\frac{B_{\theta 0}^{2}+B_{\phi 0}^{2}}{2 \mu_{0}}\right)=-\frac{B_{\theta 0}^{2}}{\mu_{0} r}
$$

que é a equação do equilíbrio em uma dimensão. Sendo assim, nas variáveis dimensionais, $\Psi(r, \theta)$ é dada por

$$
\Psi(r, \theta)=\Psi_{0}(r)+\frac{d \Psi_{0}}{d r} \Delta_{S}(r) \cos \theta
$$

onde $\Delta_{S}$ é o deslocamento de Shafranov, e é dado por

$$
\Delta_{S}(r)=\frac{1}{R_{0}} \int_{r}^{a} d r^{\prime} \frac{1}{r^{\prime} B_{\theta 0}^{2}} \int_{0}^{r^{\prime}} d r^{\prime \prime}\left(2 \mu_{0} r^{\prime \prime} \frac{d p}{d r^{\prime \prime}}-r^{\prime \prime} B_{\theta 0}^{2}\right)
$$




\section{Apêndice D}

\section{Sistema de coordenadas $\left(\hat{\boldsymbol{e}}_{\Psi}, \hat{\boldsymbol{e}}_{\|}, \hat{\boldsymbol{e}}_{\perp}\right)$}

Neste apêndice, derivamos relações envolvendo o sistema de coordenadas definido pelos versores $\left(\hat{\boldsymbol{e}}_{\Psi}, \hat{\boldsymbol{e}}_{\|}, \hat{\boldsymbol{e}}_{\perp}\right)$ em termos do sistema de coordenadas pseudo-toroidais. Em alguns casos, fazemos o inverso. Tais relações são em sua maioria operadores e versores.

\section{D.1 Versores e operadores vetoriais}

Sendo $\Psi$ a função de fluxo e $\boldsymbol{B}$ o campo magnético de equilíbrio, definem-se os seguintes versores

$$
\hat{\boldsymbol{e}}_{\Psi}=\frac{\nabla \Psi}{|\nabla \Psi|}, \quad \hat{\boldsymbol{e}}_{\|}=\frac{B}{B} \quad \text { e } \quad \hat{\boldsymbol{e}}_{\perp}=\hat{\boldsymbol{e}}_{\Psi} \times \hat{\boldsymbol{e}}_{\|}
$$

e os seguintes operadores associados a eles

$$
\nabla_{\Psi}=\hat{\boldsymbol{e}}_{\Psi} \cdot \nabla, \quad \nabla_{\|}=\hat{\boldsymbol{e}}_{\|} \cdot \nabla \quad \text { e } \quad \nabla_{\perp}=\hat{\boldsymbol{e}}_{\perp} \cdot \nabla
$$

Para tokamaks de secção circular e alta razão de aspecto, considerando a aproximação $\Psi \approx \Psi_{0}(r)$, os versores definidos por D.1 no sistema de coordenadas pseudo-toroidais assumem a forma

$$
\hat{\boldsymbol{e}}_{\Psi}=\hat{e}_{r}, \quad \hat{\boldsymbol{e}}_{\|}=\frac{\varepsilon}{q} \hat{e}_{\theta}+\hat{e}_{\phi} \quad \text { e } \quad \hat{\boldsymbol{e}}_{\perp}=-\hat{e}_{\theta}+\frac{\varepsilon}{q} \hat{e}_{\phi},
$$

em que utilizamos a aproximação $\frac{(\varepsilon / q) \hat{e}_{\theta}+\hat{e}_{\phi}}{\sqrt{\left.1+(\varepsilon / q)^{2}\right)}} \approx \frac{\varepsilon}{q} \hat{e}_{\theta}+\hat{e}_{\phi}$. A relação inversa também é 
possível:

$$
\hat{e}_{r}=\hat{\boldsymbol{e}}_{\Psi}, \quad \hat{e}_{\theta}=\frac{\varepsilon}{q} \hat{\boldsymbol{e}}_{\|}-\hat{\boldsymbol{e}}_{\perp} \quad \text { e } \quad \hat{e}_{\phi}=\hat{\boldsymbol{e}}_{\|}+\frac{\varepsilon}{q} \hat{\boldsymbol{e}}_{\perp}
$$

Utilizando B.29,

$$
\begin{gathered}
\frac{\partial \hat{e}_{r}}{\partial r}=0, \quad \frac{\partial \hat{e}_{\theta}}{\partial r}=0, \quad \frac{\partial \hat{e}_{\phi}}{\partial r}=0 \\
\frac{\partial \hat{e}_{r}}{\partial \theta}=\hat{e}_{\theta}, \quad \frac{\partial \hat{e}_{\theta}}{\partial \theta}=-\hat{e}_{r}, \quad \frac{\partial \hat{e}_{\phi}}{\partial \theta}=0 \\
\frac{\partial \hat{e}_{r}}{\partial \phi}=\cos \theta \hat{e}_{\phi}, \quad \frac{\partial \hat{e}_{\theta}}{\partial \phi}=-\sin \theta \hat{e}_{\phi}, \quad \frac{\partial \hat{e}_{\phi}}{\partial \phi}=-\cos \theta \hat{e}_{r}+\sin \theta \hat{e}_{\theta}
\end{gathered}
$$

obtêm-se os seguintes derivativos dos versores definidos em D.3

$$
\begin{array}{r}
\frac{\partial \hat{\boldsymbol{e}}_{\Psi}}{\partial r}=0, \quad \frac{\partial \hat{\boldsymbol{e}}_{\|}}{\partial r}=\frac{\varepsilon}{q^{2} R_{0}} \hat{\boldsymbol{e}}_{\|}-\frac{1}{q R_{0}} \hat{\boldsymbol{e}}_{\perp}, \quad \frac{\partial \hat{\boldsymbol{e}}_{\perp}}{\partial r}=\frac{1}{q R_{0}} \hat{\boldsymbol{e}}_{\|}+\frac{\varepsilon}{q^{2} R_{0}} \hat{\boldsymbol{e}}_{\perp} \\
\frac{\partial \hat{\boldsymbol{e}}_{\Psi}}{\partial \theta}=\frac{\varepsilon}{q} \hat{\boldsymbol{e}}_{\|}-\hat{\boldsymbol{e}}_{\perp}, \quad \frac{\partial \hat{\boldsymbol{e}}_{\|}}{\partial \theta}=-\frac{\varepsilon}{q} \hat{\boldsymbol{e}}_{\Psi}, \quad \frac{\partial \hat{\boldsymbol{e}}_{\perp}}{\partial \theta}=\hat{\boldsymbol{e}}_{\Psi} \\
\frac{\partial \hat{\boldsymbol{e}}_{\Psi}}{\partial \phi}=\cos \theta \hat{\boldsymbol{e}}_{\|}+\frac{\varepsilon}{q} \cos \theta \hat{\boldsymbol{e}}_{\perp}, \quad \frac{\partial \hat{\boldsymbol{e}}_{\|}}{\partial \phi}=-\cos \theta \hat{\boldsymbol{e}}_{\Psi}-\sin \theta \hat{\boldsymbol{e}}_{\perp}, \quad \frac{\partial \hat{\boldsymbol{e}}_{\perp}}{\partial \phi}=-\frac{\varepsilon}{q} \cos \theta \hat{\boldsymbol{e}}_{\Psi}+\sin \theta \hat{\boldsymbol{e}}_{\|},
\end{array}
$$

os quais serão úteis mais adiante.

Utilizando B.12 e D.2, obtemos a forma explícita dos operadores

$$
\nabla_{\Psi}=\frac{\partial}{\partial r}, \quad \nabla_{\|}=\frac{1}{q R_{0}} \frac{\partial}{\partial \theta}+\frac{1}{R} \frac{\partial}{\partial \phi} \quad \text { e } \quad \nabla_{\perp}=-\frac{1}{\varepsilon R_{0}} \frac{\partial}{\partial \theta}+\frac{\varepsilon}{q R} \frac{\partial}{\partial \phi} .
$$

Ao aplicar o operador $\nabla_{\Psi}, \nabla_{\|}$e $\nabla_{\perp}$ nos versores de D.3, obtemos

$$
\nabla_{\Psi} \hat{\boldsymbol{e}}_{\Psi}=0 \quad \nabla_{\Psi} \hat{\boldsymbol{e}}_{\|}=\frac{\varepsilon}{q^{2} R_{0}} \hat{\boldsymbol{e}}_{\|}-\frac{1}{q R_{0}} \hat{\boldsymbol{e}}_{\perp} \quad \text { e } \quad \nabla_{\Psi} \hat{\boldsymbol{e}}_{\perp}=\frac{1}{q R_{0}} \hat{\boldsymbol{e}}_{\|}+\frac{\varepsilon}{q^{2} R_{0}} \hat{\boldsymbol{e}}_{\perp}
$$




$$
\begin{gathered}
\nabla_{\|} \hat{\boldsymbol{e}}_{\Psi}=\left(\frac{\varepsilon}{q^{2} R_{0}}+\frac{\cos \theta}{R}\right) \hat{\boldsymbol{e}}_{\|}+\left(\frac{\varepsilon}{q} \cos \theta-\frac{1}{q R_{0}}\right) \hat{\boldsymbol{e}}_{\perp} \\
\nabla_{\|} \hat{\boldsymbol{e}}_{\|}=-\left(\frac{\varepsilon}{q^{2} R_{0}}+\frac{\cos \theta}{R}\right) \hat{\boldsymbol{e}}_{\Psi}-\frac{\sin \theta}{R} \hat{\boldsymbol{e}}_{\perp} \text { e } \nabla_{\|} \hat{\boldsymbol{e}}_{\perp}=\left(\frac{1}{q R_{0}}-\frac{\varepsilon}{q R} \cos \theta\right) \hat{\boldsymbol{e}}_{\Psi}+\frac{\sin \theta}{R} \hat{\boldsymbol{e}}_{\|} \\
\nabla_{\perp} \hat{\boldsymbol{e}}_{\Psi}=\left(\frac{\varepsilon}{q R} \cos \theta-\frac{1}{q R_{0}}\right) \hat{\boldsymbol{e}}_{\|}+\left(\frac{1}{\varepsilon R_{0}}+\frac{\varepsilon^{2}}{q^{2} R} \cos \theta\right) \hat{\boldsymbol{e}}_{\perp} \\
\nabla_{\perp} \hat{\boldsymbol{e}}_{\|}=\left(\frac{1}{q R_{0}}-\frac{\varepsilon}{q R} \cos \theta\right) \hat{\boldsymbol{e}}_{\Psi}-\frac{\varepsilon}{q R} \sin \theta \hat{\boldsymbol{e}}_{\perp} \quad \text { e } \\
\nabla_{\perp} \hat{\boldsymbol{e}}_{\perp}=-\left(\frac{1}{\varepsilon R_{0}}+\frac{\varepsilon^{2}}{q^{2} R} \cos \theta\right) \hat{\boldsymbol{e}}_{\Psi}+\frac{\varepsilon}{q R} \sin \theta \hat{\boldsymbol{e}}_{\|}
\end{gathered}
$$

onde para derivar tal resultado utilizamos D.5 e D.6.

É conveniente também obter o divergente dos versores de D.3,

$$
\boldsymbol{\nabla} \cdot \hat{\boldsymbol{e}}_{\Psi}=\left(\frac{1}{\varepsilon R_{0}}+\frac{\cos \theta}{R}\right), \quad \boldsymbol{\nabla} \cdot \hat{\boldsymbol{e}}_{\|}=-\frac{\varepsilon}{q} \frac{\sin \theta}{R} \quad \text { e } \quad \boldsymbol{\nabla} \cdot \hat{\boldsymbol{e}}_{\perp}=\frac{\sin \theta}{R}
$$

em que para isso utilizamos B.13,

$$
\boldsymbol{\nabla} \cdot \boldsymbol{B}=\frac{1}{R r}\left[\frac{\partial\left(R r B_{r}\right)}{\partial r}+\frac{\partial\left(R B_{\theta}\right)}{\partial \theta}+r \frac{\partial B_{\phi}}{\partial \phi}\right] .
$$

Sendo $\boldsymbol{v}_{i}$ o vetor definido por

$$
\boldsymbol{v}_{i}=\omega_{i} R \hat{\boldsymbol{e}}_{\perp}+u_{i} R_{0} \hat{\boldsymbol{e}}_{\|}, \quad \operatorname{com} \quad i=0,1
$$

onde $\omega_{i}=\omega_{i}(r)$ e $u_{i}=u_{i}(r, \theta)$. Utilizando D.5, D.9 e a relação vetorial A.8,

$$
\boldsymbol{\nabla} \cdot(f \boldsymbol{A})=f \boldsymbol{\nabla} \cdot \boldsymbol{A}+\boldsymbol{A} \cdot \boldsymbol{\nabla} f
$$

resulta que a divergência de $\boldsymbol{v}_{i}$ é dada por

$$
\boldsymbol{\nabla} \cdot \boldsymbol{v}_{i}=2 \omega_{i} \sin \theta-\frac{\varepsilon}{q} \frac{R_{0}}{R} u_{i} \sin \theta+\frac{1}{q} \frac{\partial u_{i}}{\partial \theta}, \quad \text { com } \quad i=0,1
$$

Também é conveniente calcular o operador 


$$
\boldsymbol{v}_{i} \cdot \nabla=\omega_{i} R \nabla_{\perp}+u_{i} R_{0} \nabla_{\|}=\left(\frac{u_{i}}{q}-\frac{\omega_{i}}{\varepsilon} \frac{R}{R_{0}}\right) \frac{\partial}{\partial \theta}
$$

e este operador atuando em $\boldsymbol{v}_{j}$ :

$$
\begin{aligned}
& \left(\boldsymbol{v}_{i} \cdot \nabla\right) \boldsymbol{v}_{j}=\omega_{i} R\left[\omega_{j} R \nabla_{\perp} \hat{\boldsymbol{e}}_{\perp}+\nabla_{\perp}\left(\omega_{j} R\right) \hat{\boldsymbol{e}}_{\perp}+u_{j} R_{0} \nabla_{\perp} \hat{\boldsymbol{e}}_{\|}+\nabla_{\perp}\left(u_{j} R_{0}\right) \hat{\boldsymbol{e}}_{\|}\right]+ \\
& u_{i} R_{0}\left[\omega_{j} R \nabla_{\|} \hat{\boldsymbol{e}}_{\perp}+\nabla_{\|}\left(\omega_{j} R\right) \hat{\boldsymbol{e}}_{\perp}+u_{j} R_{0} \nabla_{\|} \hat{\boldsymbol{e}}_{\|}+\nabla_{\|}\left(u_{j} R_{0}\right) \hat{\boldsymbol{e}}_{\|}\right] \quad \text { com } i=0,1 .
\end{aligned}
$$

Sendo assim, as componentes $\hat{\boldsymbol{e}}_{\Psi}, \hat{\boldsymbol{e}}_{\|}$e $\hat{\boldsymbol{e}}_{\perp}$ de $\left(\boldsymbol{v}_{i} \cdot \boldsymbol{\nabla}\right) \boldsymbol{v}_{j}$ são dadas por

$$
\begin{array}{r}
{\left[\left(\boldsymbol{v}_{i} \cdot \boldsymbol{\nabla}\right) \boldsymbol{v}_{j}\right]_{\Psi}=R\left[\frac{1}{q}\left(\omega_{i} u_{j}+u_{i} \omega_{j}\right)-\frac{1}{\varepsilon}\left(\frac{R}{R_{0}} \omega_{i} \omega_{j}+\frac{\varepsilon^{2}}{q^{2}} \frac{R_{0}}{R} u_{i} u_{j}\right)\right]-} \\
R_{0}\left[\frac{\varepsilon}{q}\left(\omega_{i} u_{j}+u_{i} \omega_{j}\right)+\left(\frac{\varepsilon^{2}}{q^{2}} \frac{R}{R_{0}} \omega_{i} \omega_{j}+\frac{R_{0}}{R} u_{i} u_{j}\right)\right] \cos \theta \\
{\left[\left(\boldsymbol{v}_{i} \cdot \boldsymbol{\nabla}\right) \boldsymbol{v}_{j}\right]_{\|}=\omega_{i} R\left(\frac{\varepsilon}{q} \omega_{j} \sin \theta-\frac{1}{\varepsilon} \frac{\partial u_{j}}{\partial \theta}\right)+u_{i} R_{0}\left(\omega_{j} \sin \theta+\frac{1}{q} \frac{\partial u_{j}}{\partial \theta}\right)} \\
{\left[\left(\boldsymbol{v}_{i} \cdot \nabla\right) \boldsymbol{v}_{j}\right]_{\perp}=\left[\omega_{i} R\left(\omega_{j}-\frac{\varepsilon}{q} \frac{R_{0}}{R} u_{j}\right)-u_{i} R_{0}\left(\frac{\varepsilon}{q} \omega_{j}+\frac{R_{0}}{R} u_{j}\right)\right] \sin \theta}
\end{array}
$$

\section{D.2 Ortogonalidade de funções seno-cossenoidais}

A partir das relações fundamentais,

$$
\begin{gathered}
\sin [(p \pm q) \theta]=\sin (p \theta) \cos (q \theta) \pm \sin (q \theta) \cos (p \theta) \\
\cos [(p \pm q) \theta]=\cos (p \theta) \cos (q \theta) \mp \sin (q \theta) \sin (p \theta),
\end{gathered}
$$

é possível derivar, a partir da combinação linear destas, as relações de ortogonalidade entre funções seno-cosseno, 


$$
\begin{array}{r}
\int_{0}^{2 \pi} d \theta \sin (p \theta) \cos (q \theta)=0 \\
\int_{0}^{2 \pi} d \theta \sin (p \theta) \sin (q \theta)=\pi\left(\delta_{p, q}-\delta_{p,-q}\right) \\
\int_{0}^{2 \pi} d \theta \cos (p \theta) \cos (q \theta)=\pi\left(\delta_{p, q}+\delta_{p,-q}\right),
\end{array}
$$

em que $p$ e $q$ são números inteiros quaisquer e $\delta_{i, j}$ é conhecida como função delta de Kronic, a qual assume valor 1 para $i=j$ e valor 0 caso contrário.

A relação D.15 nos permite obter a seguinte relação:

$$
\int_{0}^{2 \pi} d \theta R^{n} \sin (p \theta)=0 \quad \text { e } \quad \int_{0}^{2 \pi} d \theta R^{n} \cos (p \theta) \approx R_{0}^{n} \pi\left(2 \delta_{p, 0}+n \varepsilon \delta_{p, 1}\right)
$$

onde $R=R_{0}(1+\varepsilon \cos \theta)$ e $\varepsilon \ll 1$, de forma que

$$
R^{n}=R_{0}^{n}(1+n \varepsilon \cos \theta)+O\left(\varepsilon^{2}\right) \approx R_{0}^{n}(1+n \varepsilon \cos \theta) .
$$

\section{D.3 Operadores aplicados a funções seno-cossenoidais}

Ao aplicar os operadores definidos em D.4 em funções da forma

$$
\xi(r, \theta)_{1}=\xi_{s}(r) \sin \theta+\xi_{c}(r) \cos \theta
$$

obtemos

$$
\begin{array}{r}
\nabla_{\Psi} \xi=\frac{d \xi_{s}}{d r} \sin \theta+\frac{d \xi_{c}}{d r} \cos \theta \\
\nabla_{\|} \xi=\frac{1}{q R_{0}}\left(-\xi_{c} \sin \theta+\xi_{s} \cos \theta\right) \mathrm{e} \\
\nabla_{\perp} \xi=\frac{1}{\varepsilon R_{0}}\left(\xi_{c} \sin \theta-\xi_{s} \cos \theta\right) .
\end{array}
$$

Considerando apenas modos $n=0$ e $m=1$, se $u_{1}, \rho_{1}$ e $p_{1}$ forem da forma D.14, então utilizando D.8 temos: 


$$
\begin{array}{rr}
\boldsymbol{\nabla} \cdot \boldsymbol{v}_{\mathbf{0}}=0, \quad\left(\omega_{0}=u_{0}=0\right) & (\mathrm{ENR}) \\
\boldsymbol{\nabla} \cdot \boldsymbol{v}_{\mathbf{0}}=0, \quad\left(\omega_{0}=\frac{\varepsilon}{q} M_{T} \omega_{s 0}, \quad u_{0}=\frac{R}{R_{0}} M_{T} \omega_{s 0}\right) & (\mathrm{ETRF}) \\
\boldsymbol{\nabla} \cdot \boldsymbol{v}_{\mathbf{0}}=2 M_{\perp} \omega_{s 0} \sin \theta, \quad\left(\omega_{0}=M_{\perp} \omega_{s 0}, \quad u_{0}=0\right) & (\mathrm{EBRF})
\end{array}
$$

e

$$
\boldsymbol{\nabla} \cdot \boldsymbol{v}_{\mathbf{1}} \approx\left(2 \omega_{1}-\frac{u_{1 c}}{q}\right) \sin \theta+\frac{u_{1 s}}{q} \cos \theta
$$

onde desprezamos o termo $\frac{\varepsilon}{q} \frac{R_{0}}{R} u_{1} \sin \theta$, por ser de ordem $O\left(\varepsilon^{2}\right)$.

Ao aplicar D.9 em $\xi$, dado por D.14, utilizando também D.15, obtém-se

$$
\begin{array}{rr}
\left(\boldsymbol{v}_{\mathbf{0}} \cdot \boldsymbol{\nabla}\right) \xi_{1}=0, & (\mathrm{ENR}) \\
\left(\boldsymbol{v}_{\mathbf{0}} \cdot \boldsymbol{\nabla}\right) \xi_{1}=0, & (\mathrm{ETRF}) \\
\left(\boldsymbol{v}_{\mathbf{0}} \cdot \boldsymbol{\nabla}\right) \xi_{1}=\frac{1}{q} \frac{R}{R_{0}} M_{\perp} \omega_{s 0}\left(\xi_{c} \sin \theta-\xi_{s} \cos \theta\right), & (\mathrm{EBRF}) .
\end{array}
$$

De forma similar, considerando $\frac{\partial \rho_{0}}{\partial \theta}$ e $\frac{\partial p_{0}}{\partial \theta}$, descrito no capítulo 4,

$$
\begin{aligned}
& \frac{\partial \rho_{0}}{\partial \theta}=0 \quad \text { e } \quad \frac{\partial p_{0}}{\partial \theta}=0, \quad(\mathrm{ENR}) \\
& \left.\frac{\partial \rho_{0}}{\partial \theta}=2 \gamma M_{T}^{2} \frac{R}{R_{0}} \rho_{0} \varepsilon \sin \theta \quad \text { e } \quad \frac{\partial p_{0}}{\partial \theta}=\frac{\omega_{s 0}^{2} R_{0}^{2}}{\gamma} \frac{\partial \rho_{0}}{\partial \theta} \quad \text { (ETRF }\right) \\
& \frac{\partial \rho_{0}}{\partial \theta}=2 \alpha \frac{R_{0}}{R} \rho_{0} \varepsilon \sin \theta \quad \text { e } \quad \frac{\partial p_{0}}{\partial \theta}=2 \omega_{s 0}^{2} R_{0}^{2} \frac{\beta}{\alpha} \frac{\partial \rho_{0}}{\partial \theta}, \quad(\mathrm{EBRF})
\end{aligned}
$$

obtém-se

$$
\begin{array}{r}
\left(\boldsymbol{v}_{\mathbf{1}} \cdot \boldsymbol{\nabla}\right) \rho_{0}=0, \quad(\mathrm{ENR}) \\
\left(\boldsymbol{v}_{\mathbf{1}} \cdot \boldsymbol{\nabla}\right) \rho_{0}=\frac{1}{\varepsilon}\left(\frac{\varepsilon}{q} u_{1}-\frac{R}{R_{0}} \omega_{1}\right) \frac{\partial \rho_{0}}{\partial \theta}, \quad(\mathrm{ETRF} / \mathrm{EBRF}) .
\end{array}
$$


Ao considerar apenas modos $m=1$, isto é, ao desprezar termos da forma $\sin (p \theta)$ e $\cos (p \theta), \operatorname{com} p \neq \pm 1$, podemos escrever D.20 como

$$
\begin{array}{rr}
\left(\boldsymbol{v}_{\mathbf{1}} \cdot \boldsymbol{\nabla}\right) \rho_{0}=0, & (\mathrm{ENR}) \\
\left(\boldsymbol{v}_{\mathbf{1}} \cdot \boldsymbol{\nabla}\right) \rho_{0}=-2 \gamma M_{T}^{2} \frac{R^{2}}{R_{0}^{2}} \omega_{1} \rho_{0} \sin \theta, & (\mathrm{ETRF}) \\
\left(\boldsymbol{v}_{\mathbf{1}} \cdot \boldsymbol{\nabla}\right) \rho_{0}=-2 \omega_{1} \rho_{0} \sin \theta, & (\mathrm{EBRF}) .
\end{array}
$$

Utilizando as relações D.10 - D.13 e D.18 -D.20, as funções

$$
\begin{array}{r}
\boldsymbol{F}=\left(\boldsymbol{v}_{\mathbf{0}} \cdot \boldsymbol{\nabla}\right) \boldsymbol{v}_{\mathbf{1}}+\left(\boldsymbol{v}_{\mathbf{1}} \cdot \boldsymbol{\nabla}\right) \boldsymbol{v}_{\mathbf{0}}+\frac{\rho_{1}}{\rho_{0}}\left(\boldsymbol{v}_{\mathbf{0}} \cdot \boldsymbol{\nabla}\right) \boldsymbol{v}_{\mathbf{0}}, \quad \boldsymbol{F}=F_{\Psi} \hat{\boldsymbol{e}}_{\Psi}+F_{\|} \hat{\boldsymbol{e}}_{\|}+F_{\perp} \hat{\boldsymbol{e}}_{\perp} \\
G=\left(\boldsymbol{v}_{\mathbf{0}} \cdot \boldsymbol{\nabla}\right) \rho_{1}+\left(\boldsymbol{v}_{\mathbf{1}} \cdot \boldsymbol{\nabla}\right) \rho_{0}+\rho_{1} \boldsymbol{\nabla} \cdot \boldsymbol{v}_{\mathbf{0}} \quad \mathrm{e} \\
H=\left(\boldsymbol{v}_{\mathbf{0}} \cdot \boldsymbol{\nabla}\right) p_{1}+\frac{1}{\gamma}\left(\boldsymbol{v}_{\mathbf{1}} \cdot \boldsymbol{\nabla}\right)\left(\omega_{s 0}^{2} R_{0}^{2} \rho_{0}\right)+\gamma p_{1} \boldsymbol{\nabla} \cdot \boldsymbol{v}_{\mathbf{0}}
\end{array}
$$

onde $F_{\Psi}=\hat{\boldsymbol{e}}_{\Psi} \cdot \boldsymbol{F}, F_{\|}=\hat{\boldsymbol{e}}_{\|} \cdot \boldsymbol{F}$ e $F_{\perp}=\hat{\boldsymbol{e}}_{\perp} \cdot \boldsymbol{F}$, podem ser escritas como:

- Para equilíbrio sem rotação - ENR

$$
F_{\|}=F_{\perp}=G=H=0
$$

- Para equilíbrio com rotação toroidal - ETRF

$$
\begin{array}{r}
F_{\|}=2 M_{T} \omega_{s 0} \omega_{1} R_{0} \sin \theta, \quad F_{\perp}=-\frac{1}{2} R_{0} M_{T} \omega_{s 0}\left(2 u_{1 s}+M_{T} \omega_{s 0} \frac{\rho_{1 s}}{\rho_{0}}\right)+O(\neq \cos \theta), \\
G=-2 \gamma M_{T}^{2} \omega_{1} \rho_{0} \sin \theta \quad \text { e } H=-2 M_{T}^{2} \omega_{s 0}^{2} \omega_{1} R_{0}^{2} \rho_{0} \sin \theta, \quad(\mathrm{D} .
\end{array}
$$

- Para equilíbrio com rotação binormal - EBRF 


$$
\begin{array}{r}
F_{\|} \approx=\frac{M_{\perp} \omega_{s 0} R_{0}}{\varepsilon}\left[\left(2 \frac{\varepsilon^{2}}{q} \omega_{1}+u_{1 c}\right) \sin \theta-u_{1 s} \cos \theta\right], \\
F_{\perp}=-\frac{1}{2} R_{0} M_{\perp} \omega_{s 0}\left(2 \frac{\varepsilon}{q} u_{1 s}-M_{\perp} \omega_{s 0} \frac{\rho_{1 s}}{\rho_{0}}\right)+O(\neq \cos \theta), \\
G=\frac{1}{q} \frac{R}{R_{0}} M_{\perp} \omega_{s 0}\left[\left(\rho_{1 c}-2 \gamma q \frac{R_{0}}{R} \frac{\omega_{1}}{\omega_{s 0}} M_{\perp} \rho_{0}\right) \sin \theta-\rho_{1 s} \cos \theta\right] \mathrm{e} \\
H=\frac{1}{q} \frac{R}{R_{0}} M_{\perp} \omega_{s 0}\left[\left(p_{1 c}-2 q \frac{R_{0}}{R} M_{\perp} \omega_{s 0} \omega_{1} \rho_{0}\right) \sin \theta-p_{1 s} \cos \theta\right] .
\end{array}
$$




\section{Apêndice E}

\section{Cálculo do determinante do capítulo 6}

O determiante é dado por

$$
\left|\begin{array}{ccccccc}
-i \Omega & -1 & 0 & \frac{M_{\perp}^{2}}{2} & 0 & -\frac{\varepsilon}{q} M_{\perp} & 0 \\
2 \frac{\varepsilon}{q} M_{\perp} & 0 & -\frac{1}{q} & 0 & 0 & -i \Omega & \frac{M_{\perp}}{\varepsilon} \\
0 & \frac{1}{q} & 0 & 0 & 0 & \frac{-M_{\perp}}{\varepsilon} & -i \Omega \\
2(1-\alpha) & 0 & 0 & -i \Omega & \frac{M_{\perp}}{\varepsilon} & 0 & -\frac{1}{q} \\
0 & 0 & 0 & \frac{-M_{\perp}}{\varepsilon} & -i \Omega & \frac{1}{q} & 0 \\
2(1-\beta) & -i \Omega & \frac{M_{\perp}}{\varepsilon} & 0 & 0 & 0 & -\frac{1}{q} \\
0 & \frac{-M_{\perp}}{\varepsilon} & -i \Omega & 0 & 0 & \frac{1}{q} & 0
\end{array}\right|=0
$$

Para simplificar a resolução da equação E.1 é conveniente seguir os seguintes passos:
1) $L_{1} \times(-2 q)$
2) $C_{1} \times(-i / 2)$
3) $L_{2} \times i q$
4) $C_{2} \times(-1)$
5) $L_{3} \times(-q)$
6) $C_{3} \times i$
7) $L_{4} \times(-i q)$
8) $C_{4} \times(-1 / q)$
9) $L_{5} \times q$
10) $C_{5} \times(i / q)$
11) $L_{6} \times(-i q)$
12) $L_{7} \times q$
13) $C_{7} \times(-i)$
14) Define-se $m_{\perp}=M_{\perp} / \varepsilon$, 
onde $L_{k}$ e $C_{k}$ é a linha $k$ e a coluna $k$, respectivamente $(k=1,2, \ldots, 7)$. A equação resultante é dada por:

$\left|\begin{array}{ccccccc}q \Omega & -2 q & 0 & \varepsilon^{2} m_{\perp}^{2} & 0 & 2 \varepsilon^{2} m_{\perp} & 0 \\ \varepsilon^{2} m_{\perp} & 0 & 1 & 0 & 0 & q \Omega & q m_{\perp} \\ 0 & 1 & 0 & 0 & 0 & q m_{\perp} & q \Omega \\ (\alpha-1) q & 0 & 0 & \Omega & m_{\perp} & 0 & 1 \\ 0 & 0 & 0 & m_{\perp} & \Omega & 1 & 0 \\ (\beta-1) q & q \Omega & q m_{\perp} & 0 & 0 & 0 & 1 \\ 0 & q m_{\perp} & q \Omega & 0 & 0 & 1 & 0 \\ & & & & & & \end{array}\right|=0$

Ao utilizar o programa Maple 9 para resolver E.2, obtemos as seguintes soluções

$$
\begin{array}{r}
\Omega^{2}=m_{\perp}^{2}, \\
\Omega^{2}=\left(1-\beta+m_{\perp}^{2}\right)+\frac{1}{q^{2}}+\sqrt{(1-\beta)^{2}+\frac{4 m_{\perp}^{2}}{q^{2}}} \mathrm{e} \\
\Omega^{2}=\left(1-\beta+m_{\perp}^{2}\right)+\frac{1}{q^{2}}-\sqrt{(1-\beta)^{2}+\frac{4 m_{\perp}^{2}}{q^{2}}} .
\end{array}
$$

Para o caso com rotação, ou seja, $\beta=1$ e $m_{\perp} \neq 0$, temos

$$
\Omega^{2}=m_{\perp}^{2}, \quad \Omega^{2}=m_{\perp}^{2}+\frac{1}{q^{2}}+\frac{2 m_{\perp}}{q} \quad \text { e } \quad \Omega^{2}=m_{\perp}^{2}+\frac{1}{q^{2}}-\frac{2 m_{\perp}}{q},
$$

já para o limite sem rotação, ou seja, $\beta=0$ e $m_{\perp}=0$, 


$$
\Omega^{2}=0, \quad \Omega^{2}=2+\frac{1}{q^{2}} \quad \text { e } \quad \Omega^{2}=\frac{1}{q^{2}} .
$$




\section{Apêndice F}

\section{Lista de símbolos e siglas utilizados ao longo da dissertação}

\section{F.1 Lista dos principais símbolos matemáticos}

$a$ - Raio menor da coluna de plasma no tokamak

$\boldsymbol{B}$ - Campo magnético

$B_{\theta}$ - Campo magnético poloidal

$B_{\phi}$ - Campo magnético toroidal

$c_{s_{0}}$ - Velocidade do som no plasma

$D$ - Coeficiente de difusão

$\frac{D}{D t}$ - Derivada lagrangeana

$D_{\theta}$ - Operador definido na página 35

$\boldsymbol{E}$ - Campo elétrico

$\boldsymbol{E}^{\prime}$ - Campo elétrico no referencial do plasma

$\boldsymbol{J}$ - Densidade de corrente

$M_{\perp}$ - Número de Mach binormal (Razão entre a velocidade de rotação binormal de equilíbrio e a velocidade do som no plasma)

$m_{\perp}$ - Razão entre $M_{\perp}$ e $\varepsilon\left(m_{\perp}=M_{\perp} / \varepsilon\right)$

$M_{T}$ - Número de Mach toroidal (Razão entre a velocidade de rotação toroidal de equilíbrio e a velocidade do som no plasma) 
$\omega_{s 0}$ - Razão entre a velocidade do som no plasma e o raio maior $\left(\omega_{s 0}=c_{s_{0}} / R_{0}\right)$

$p$ - Pressão cinética do plasma

$q$ - Fator de segurança

$r$ - Raio menor em uma posição arbitrária da coluna de plasma no tokamak

$R$ - Raio maior em uma posição arbitrária da coluna de plasma no tokamak $(R=$ $\left.R_{0}+r \cos \theta\right)$

$R_{0}$ - Raio maior do eixo magnético da coluna de plasma no tokamak

$T$ - Temperatura do plasma

$u_{i}$ - Componente paralela (ao campo mangético) da velocidade $(i=0$ - no equilíbrio, $i=1$ - na perturbação)

$\boldsymbol{v}$ - Velocidade do plasma

$\boldsymbol{v}_{d}$ - Velocidade de deriva do plasma

$v_{T}$ - Velocidade toroidal de equilíbrio

$\beta$ - Razão entre a pressão cinética e a pressão magnética

$\Delta_{S}$ - Deslocamento de Shafranov

$\Delta^{*}$ - Operador elíptico (definido na página 29)

$\epsilon$ - Razão entre $a$ e $R_{0}\left(\epsilon=a / R_{0}\right)$.

$\varepsilon$ - Razão entre $r$ e $R_{0}\left(\varepsilon=r / R_{0}\right)$.

$\Phi$ - Potêncial eletrostático do plasma

$\phi$ - Ângulo toroidal

$\gamma$ - Razão entre as capacidades térmicas a pressão e a volume constantes $\left(\gamma=C_{P} / C_{V}\right)$

$\jmath(A, B)$ - Jacobiano das funções arbitrárias $A$ e $B$.

$\lambda_{D}$ - Comprimento de Debye

$\eta$ - Resistividade elétrica do plasma

$\theta$ - Ângulo poloidal

$\rho$ - Densidade de massa

$\bar{\rho}_{0}$ - Média sobre uma superfície magnética da densidade de equilíbrio.

$\tau$ - Densidade de carga elétrica do plasma 
$\omega$ - Frequência de oscilação dos modos

$\Omega$ - Frequência normalizada adimensional $\left(\Omega=\omega / \omega_{s 0}\right)$

$\omega_{i}$ - Componente binormal da velocidade ( $i=0$ - no equilíbrio, $i=1$ - na perturbação)

$\omega_{\perp}$ - Velocidade angular binormal de equilíbrio (o valor é próximo da velocidade angular poloidal de equilíbrio)

$\omega_{p e}$ - Frequência de plasma

$\omega_{g, n r}$ - Frequência não normalizada dos modos acústicos geodésicos sem rotação.

$\omega_{z, \text { nr }}$ - Frequência não normalizada dos fluxos zonais sem rotação.

$\Omega_{g, \text { tr }}$ - Frequência normalizada dos modos acústicos geodésicos com rotação toroidal.

$\Omega_{z, \operatorname{tr}}$ - Frequência normalizada dos fluxos zonais com rotação toroidal.

$\omega_{T}$ - Velocidade angular toroidal de equilíbrio

$\Psi$ - Superfíe magnética

$\xi_{0}$ - Representação de grandezas física no estado de equilíbrio de forma genérica (a letra $\xi$ é utilizada para representar $p, \rho, \boldsymbol{v}, \Phi$ e $\boldsymbol{E}$ )

$\xi_{1}$ - Representação de grandezas física no estado perturbado de forma genérica (a letra $\xi$ é utilizada para representar $p, \rho, \boldsymbol{v}, \Phi$ e $\boldsymbol{E}$ )

$\frac{d}{d \Psi}$ - Derivada total com relação a $\Psi$

\section{F.2 Lista de siglas}

EBRF - Equilíbrio com rotação binormal

ENR - Equilíbrio sem rotação

ER - Equilíbrio com rotação

ETRF - Equilíbrio com rotação toroidal

GAM - Modos acústicos geodésicos

ITER - Reator termonuclear experimental internacional

Modos H - Modos de alto confinamento

Modos L - Modos de baixo confinamento

ZF - Fluxos zonais 